\title{
Employ sensor fusion techniques for determining aircraft attitude and position information
}

Jason A. Jarrell

West Virginia University

Follow this and additional works at: https://researchrepository.wvu.edu/etd

\section{Recommended Citation}

Jarrell, Jason A., "Employ sensor fusion techniques for determining aircraft attitude and position information" (2008). Graduate Theses, Dissertations, and Problem Reports. 1908.

https://researchrepository.wvu.edu/etd/1908

This Thesis is protected by copyright and/or related rights. It has been brought to you by the The Research Repository @ WVU with permission from the rights-holder(s). You are free to use this Thesis in any way that is permitted by the copyright and related rights legislation that applies to your use. For other uses you must obtain permission from the rights-holder(s) directly, unless additional rights are indicated by a Creative Commons license in the record and/ or on the work itself. This Thesis has been accepted for inclusion in WVU Graduate Theses, Dissertations, and Problem Reports collection by an authorized administrator of The Research Repository @ WVU. For more information, please contact researchrepository@mail.wvu.edu. 


\title{
Employ Sensor Fusion Techniques for Determining Aircraft Attitude and Position Information
}

\author{
Jason A. Jarrell \\ Thesis submitted to the \\ College of Engineering and Mineral Resources \\ at West Virginia University \\ in partial fulfillment of the requirements \\ for the degree of \\ Master of Science \\ in \\ Aerospace Engineering
}

Marcello R. Napolitano, Ph.D., Chair

$\mathrm{Yu} \mathrm{Gu}, \mathrm{Ph} . \mathrm{D}$.

Powsiri Klinkhachorn, Ph.D.

Brad Seanor, Ph.D.

Department of Mechanical and Aerospace Engineering

Morgantown, WV

2008

Keywords: Sensor Fusion, Data Fusion, Inertial Navigation System, Kalman Filter 


\section{Abstract \\ Employ Sensor Fusion Techniques for Determining Aircraft Attitude and Position Information}

\section{Jason Andrew Jarrell}

Inertial Navigation Systems (INS) with the level of precision needed for Unmanned Aerial Vehicles (UAV) can easily cost more than the vehicle itself. This drastically increases the amount of aircraft power consumption and payload weight that drives the need for a low cost solution. This can be achieved through the use of sensor fusion techniques on low cost accelerometers and gyroscopes fused with Global Positioning System (GPS) data. In this paper, existing GPS and Inertial Measurement Unit (IMU) flight data is fused with the use of both an Kalman filter (KF) and Extended Kalman filter (EKF) methods for a more accurate estimate of the aircraft attitude, velocity, and position eliminating the need for the high cost attitude sensors. A simulation study shows that four sensor fusion methods verifying that an improvement of position, velocity, and attitude can be achieved using low-cost sensors. The first method incorporates a six state KF that corrects INS/GPS position and velocity errors. The second method features the GPS to estimate attitude parameters, which in turn uses in an EKF to correct INS attitude values. With this method, improved attitude values are obtained without the calculation of the full INS state; such that the INS position and velocity are not required, reducing the computational load. The third method uses only the GPS and INS position and velocity to correct for the errors in the full state of the INS also using an EKF. Finally, the last method combines the GPS attitude of the second method and the error reduction of the third method to further decrease the error in the velocity, position, and attitude of the system. The simulation results illustrate that all of the methods tested provide performance improvement to the system, and could be implemented in real-time on a UAV for accurate navigation parameters. 


\section{Acknowledgements}

First and foremost I would like to thank Dr. Marcello Napolitano and the whole research group (Brad, Gu, Srik, Giampy) for providing the opportunity and means to work in such an outstanding learning environment. The knowledge and skills I have developed along with the experiences over the past two years have been the most beneficial periods of my time in academia.

I would like to dedicate this thesis to my family who have stood by, supported, and influenced my life in so many ways up to this point in my life. Your guidance, patience, love and support was needed and I could not have completed this paper without you. I want to thank my parents and grandparents who have always been there for advice, pushed my to work harder, correcting me when I did stupid things, and of course bringing me into the world. Gary and Sarah, I want to thank you for your patience and resilience during the completion of this paper, and for always being they're for me, congratulations on a long and happy life together. Finally to my dear sister Julie, one of my closest friends, you have and will continue to be one of the most influential voices in my life, I am extremely proud of you, and could not have chosen a better husband for you than Brad, congratulations.

Finally I would like to thank all of my friends, new and old, whom I have the pleasure to spend the past few years working and playing with. This period in my life will always be remembered as one of the most fun and memorable times and will not be forgotten. LETS GO MOUNTAINEERS!!!! 


\section{Table of Contents}

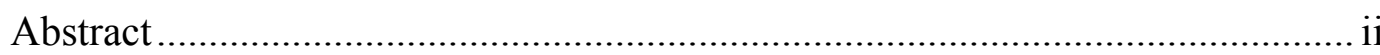

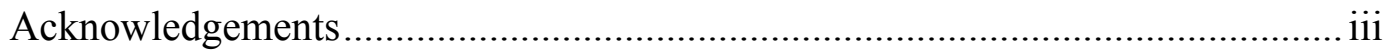

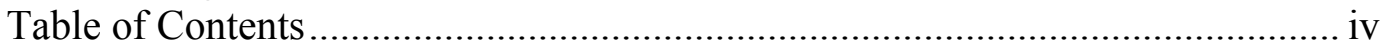

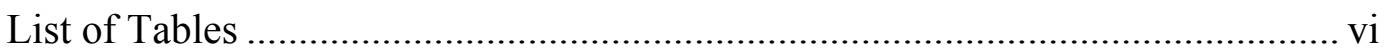

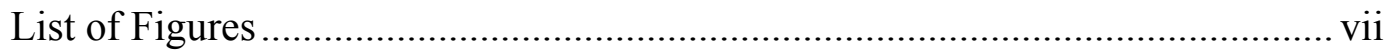

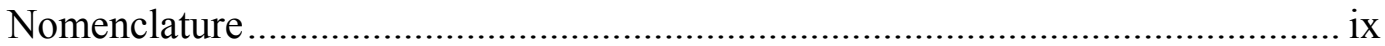

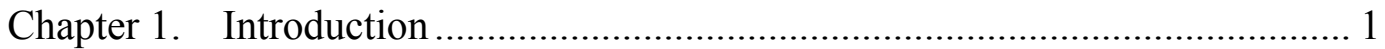

1.1. Problem Definition......................................................................... 1

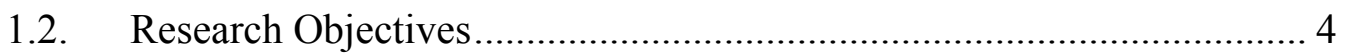

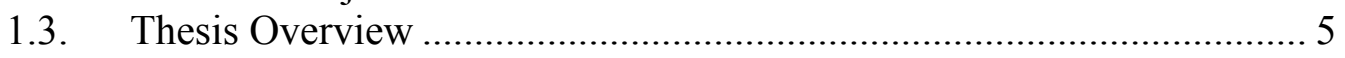

Chapter 2. Literature Review ………………………..................................... 7

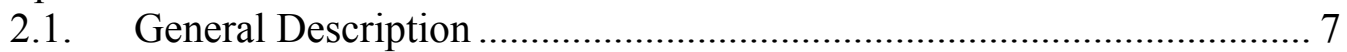

2.2. Kalman Filter Methods ....................................................................... 8

2.2.1. Simple Kalman Filter ....................................................................... 8

2.2.2. Extended Kalman Filter ................................................................. 9

2.2.3. Unscented Kalman Filter .............................................................. 9

2.3. Sensor Fusion Applications ........................................................... 10

2.3.1. Naval Applications.................................................................. 10

2.3.2. Aerospace Applications ……………………............................... 12

2.3.3. Ground Vehicle Applications …………….................................. 15

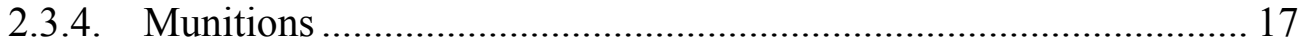

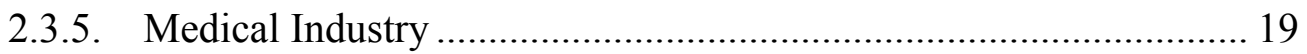

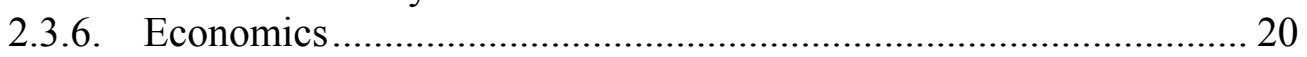

Chapter 3. Theoretical Background …………………..................................... 22

3.1. Overview of Theoretical Approach ................................................... 22

3.2. Coordinate Frames of Reference ………………….......................... 22

3.2.1. Coordinate Frame Descriptions .................................................... 22

3.2.2. Reference Frame Conversions ………………............................. 27

3.3. Inertial Navigation System (INS) ………………............................. 30

3.3.1. INS Description ...................................................................... 30

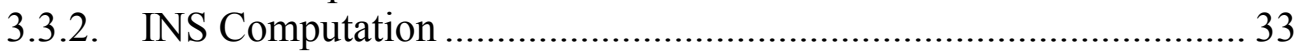

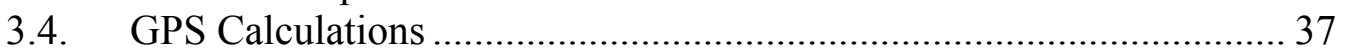

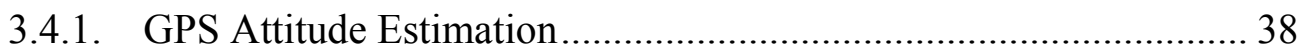

3.5. Kalman Filter ………………………………........................... 42

3.5.1. Introduction to Kalman Filtering ……………............................. 42

3.5.2. Extended Kalman Filter .............................................................. 46

Chapter 4. Experimental Procedures................................................................... 49

4.1. Overview of Experimental Procedures .................................................. 49

4.2. WVU YF-22 IMU/GPS/Vertical Gyro Sensor Fusion ........................... 49

4.2.1. Hardware Used for the WVU YF-22 Attitude Improvement .......... 49

4.2.2. Limitations/Corrections to the WVU Formation Flight Hardware

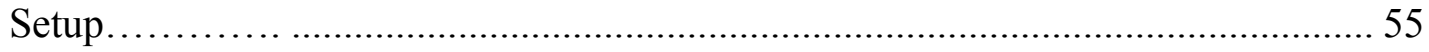


4.2.3. Software Used for the Integration of GPS/INS ........................... 57

Chapter 5. Simulation Results and Discussion ............................................... 77

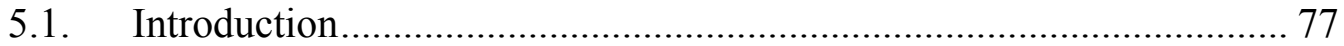

5.2. GPS/IMU/Vertical Gyro Sensor Fusion Simulation Results ................ 80

5.3. GPS/GPS Attitude/IMU Sensor Fusion Simulation Results................ 85

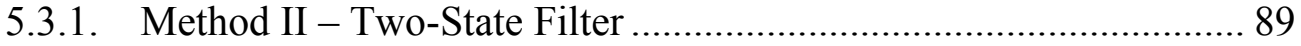

5.4. GPS/IMU Sensor Fusion Simulation Results .................................. 92

5.5. Method IV - Combination of Method II and III ............................... 93

5.6. Method Comparisons and Discussions ............................................ 94

5.7. Computational Workload Analysis ................................................ 97

Chapter 6. Conclusions and Recommendations........................................... 101

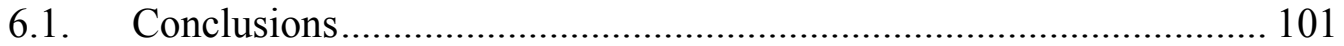

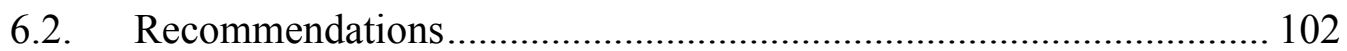

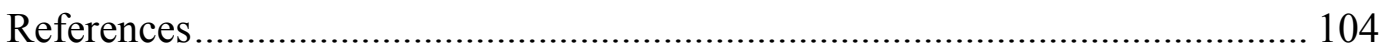

AppendixT A Method II - Additional Plots and Error Analysis ....................... A-1

Appendix B Method III - Additional Plots and Error Analysis ....................... B-1

Appendix C Method IV - Additional Plots and Error Analysis ....................... C-1 


\section{List of Tables}

Table 3-1: WGS84 Parameters .................................................................................... 24

Table 3-2: ECEF Rectangular to Geodetic Coordinate Conversion ${ }^{45}$............................. 28

Table 4-1: WVU YF-22 Test Vehicles/Sensor-Componant Package ............................. 50

Table 4-2: Sensor Specifications and Component Mounting ......................................... 51

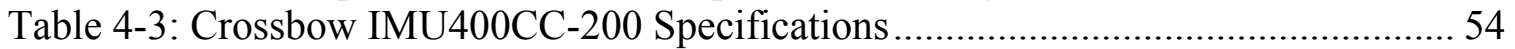

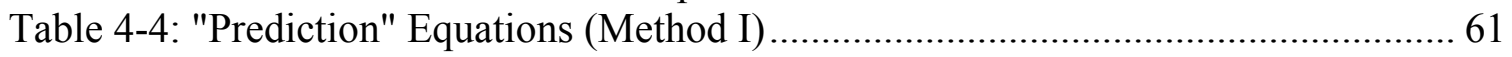

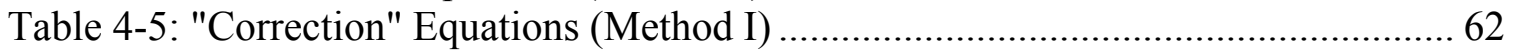

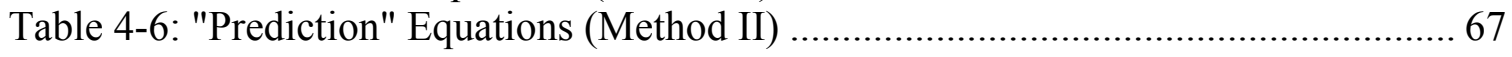

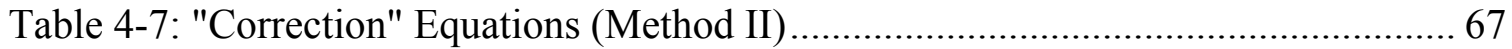

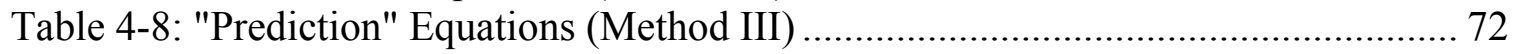

Table 4-9: "Correction" Equations (Method III) .............................................................. 72

Table 4-10: "Prediction" Equations (Method IV) .............................................................. 75

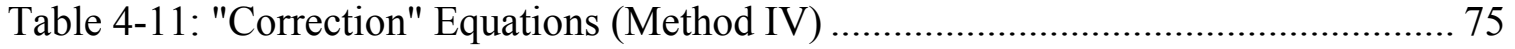

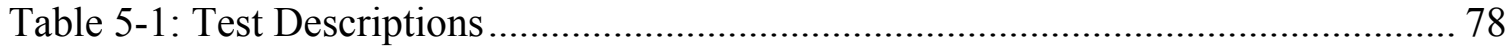

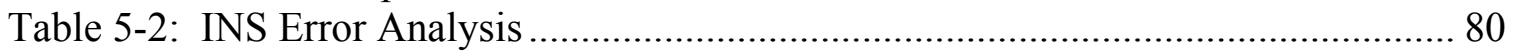

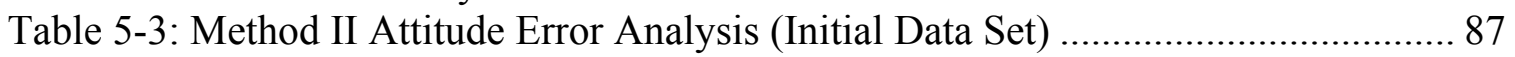

Table 5-4: Method II- Attiude Error Analysis (Validation Data Set 1) ........................... 88

Table 5-5: Method II Attitude Error Analysis (Validation Data Set 2) .......................... 88

Table 5-6: Method II Attitude Error Analysis (2 State)............................................. 91

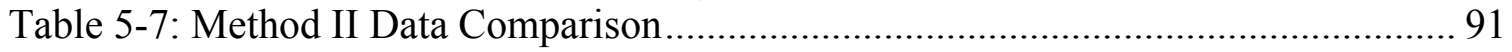

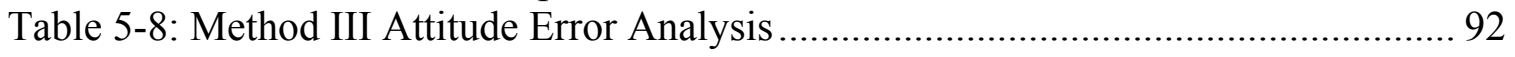

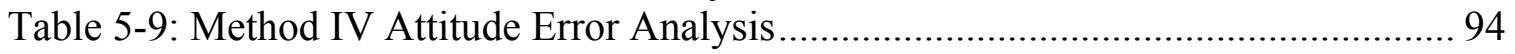

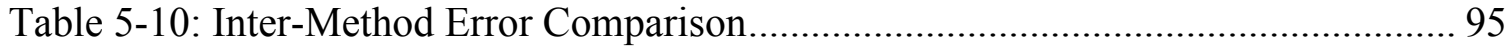

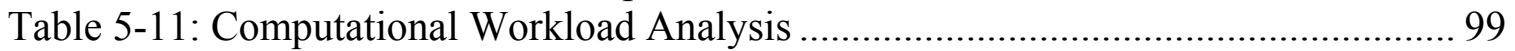




\section{List of Figures}

Figure 1.1: WVU YF-22 Research Test-beds ............................................................ 1

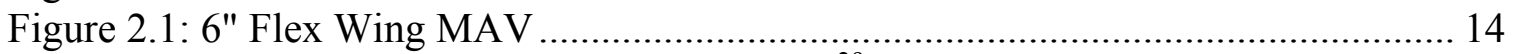

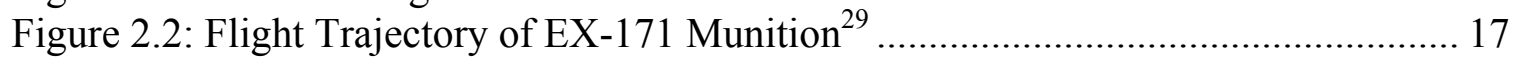

Figure 2.3: SiIMU02 IMU Developed by BAE Systems for ERGM Research............... 18

Figure 3.1: ECI and ECEF Coordinate Frame Representation ...................................... 23

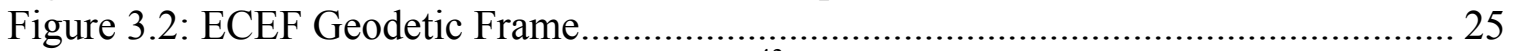

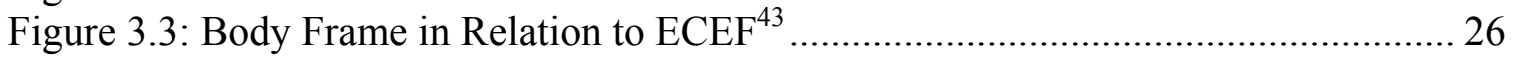

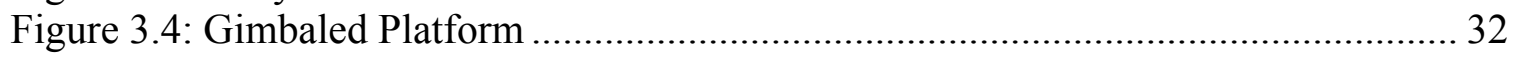

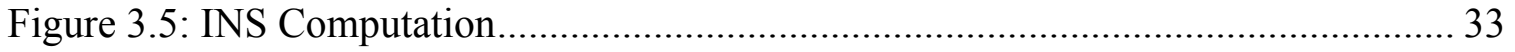

Figure 3.6. INS Data vs. Vertical Gyro Data ................................................................. 36

Figure 3.7. INS Data vs. Vertical Gyro Data (Magnified)............................................. 36

Figure 3.8: INS Data vs GPS Comparison............................................................... 37

Figure 3.9: GPS Heading Angle ............................................................................ 38

Figure 3.10: Rotation about the z-Axis to Align the ECEF Coordinate Frame with the

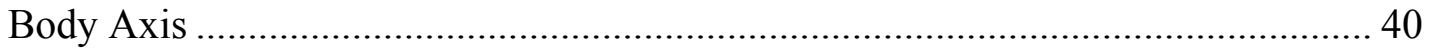

Figure 3.11: GPS vs Vertical Gyro Attitude.............................................................. 41

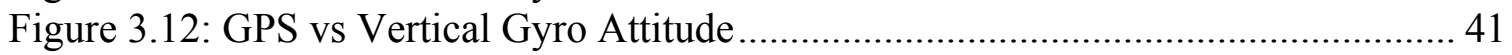

Figure 3.13: Kalman Filter Sequence ..................................................................... 42

Figure 3.14: Kalman Filter Progression of States and Covariance Matrix ..................... 46

Figure 3.15: Extended Kalman Filter (EKF) Sequence ............................................. 47

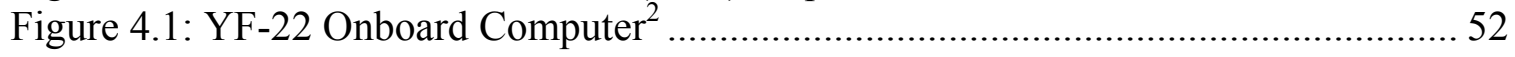

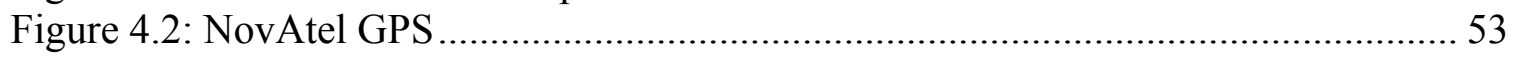

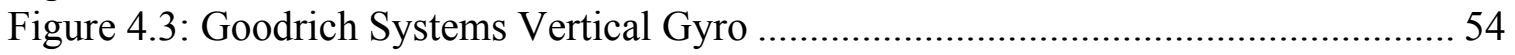

Figure 4.4: IMU vs. GPS Measurement Acquisition Rate............................................. 55

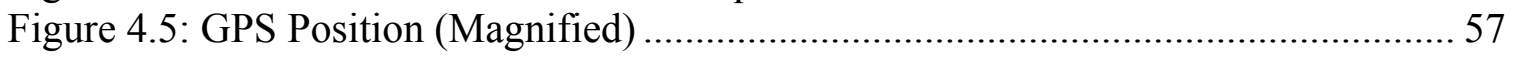

Figure 4.6: GPS Position - Instantaneous Signal Loss .............................................. 57

Figure 4.7: Steady State Time Period for Variance Calculation..................................... 59

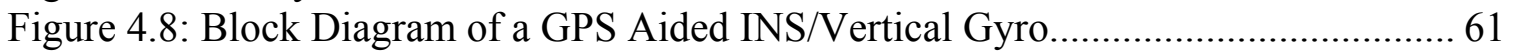

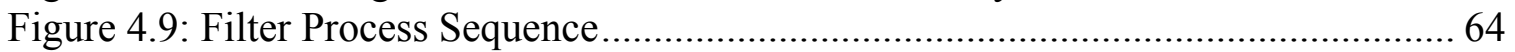

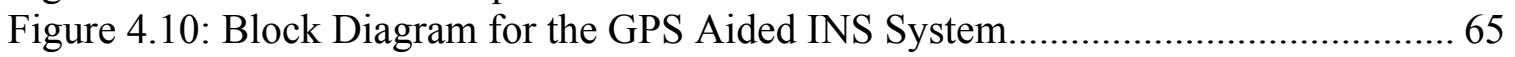

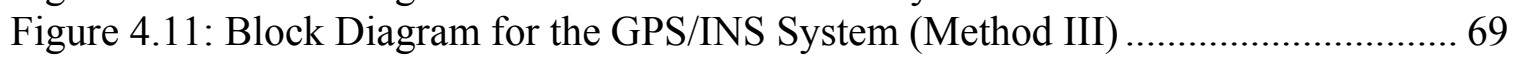

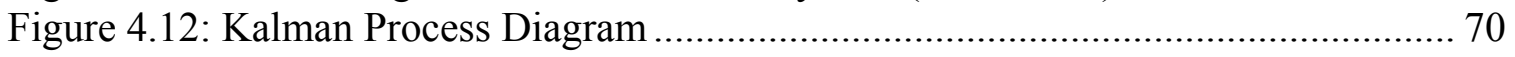

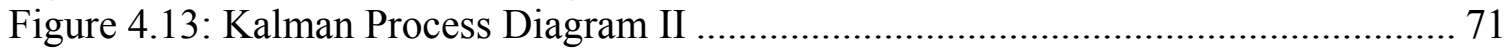

Figure 4.14: Block Diagram GPS Aided Attitude/DCM System .................................. 74

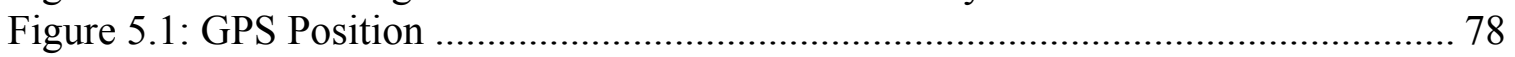

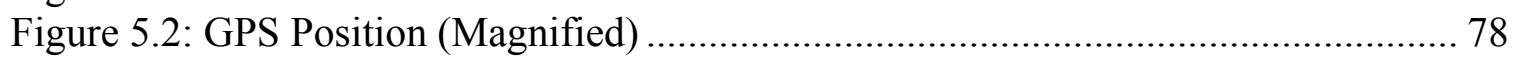

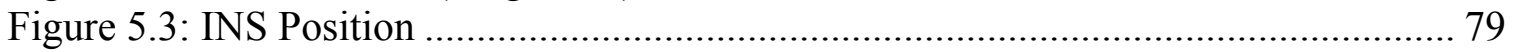

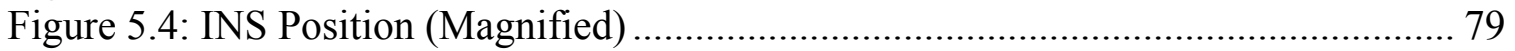

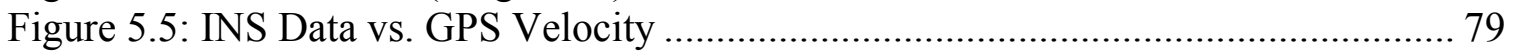

Figure 5.6: Vertical Gyro vs. INS Roll and Pitch Angle ............................................ 80

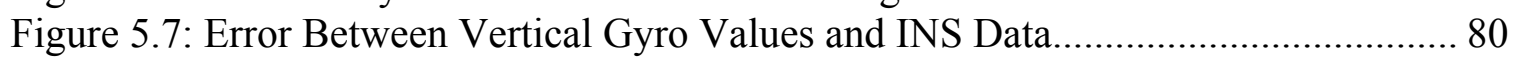

Figure 5.8: GPS vs. GPS-INS Velocity Plot (6 State) ................................................. 81

Figure 5.9: GPS vs. GPS-INS Velocity Plot (Magnified - 6 State) ............................... 81 
Figure 5.10: GPS vs. GPS-INS Data Position Plot (6 State) ...................................... 82

Figure 5.11: GPS vs. GPS-INS Data Position Plot (Magnified - 6 State) ....................... 82

Figure 5.12: GPS vs. Filtered Data Position Plot (3 State) .......................................... 83

Figure 5.13: GPS vs. Filtered Data Position Plot (Magnified - 3 State) ........................... 83

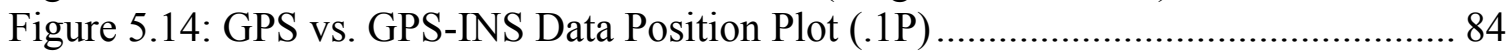

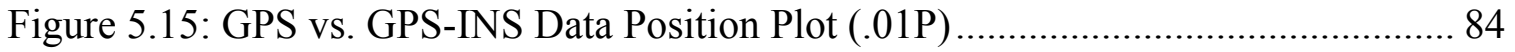

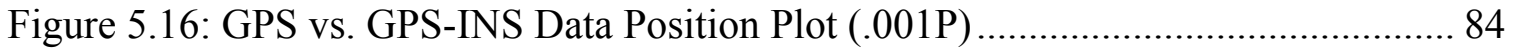

Figure 5.17: GPS vs. GPS-INS Data Position Plot (.0001P) ....................................... 84

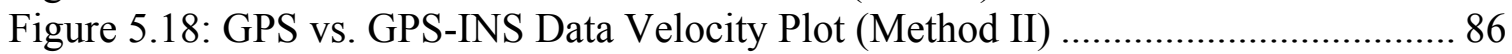

Figure 5.19: GPS vs. GPS-INS Data Position Plot (Method II) ..................................... 86

Figure 5.20: Vertical Gyro/INS Data/GPS-INS Filtered Data Roll Angle Comparison

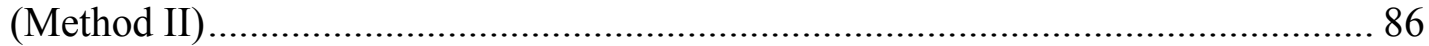

Figure 5.21: Vertical Gyro/INS Data/GPS-INS Data Roll Angle Comparison (Magnified Method II) .......................................................................................... 86

Figure 5.22: Vertical Gyro/INS Data/GPS-INS Filtered Data Roll Angle Comparison

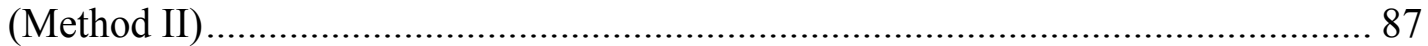

Figure 5.23: Vertical Gyro/INS Data/GPS-INS Filtered Data Roll Angle Comparison

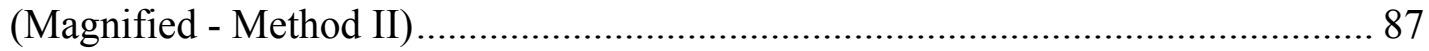

Figure 5.24: Validation Data Set 2 - Roll and Pitch Actual Error (Method II) ................ 89

Figure 5.25: Vertical Gyro/INS Data/GPS-INS Filtered Data Roll Angle Comparison .. 90

Figure 5.26: Vertical Gyro/INS Data/GPS-INS Filtered Data Roll Angle Comparison (Magnified - Method II - 2 State Filter) ................................................................ 90

Figure 5.27: Vertical Gyro/INS Data/GPS-INS Filtered Data Pitch Angle Comparison. 90

Figure 5.28: Vertical Gyro/INS Data/GPS-INS Filtered Data Pitch Angle Comparison

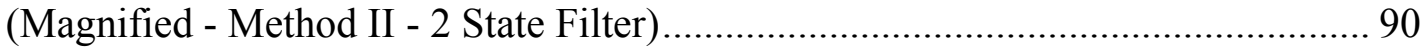

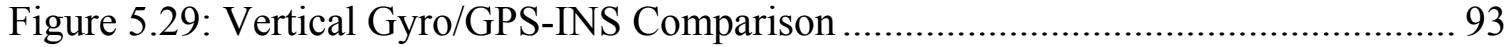

Figure 5.30: GPS-INS Data/Vertical Gyro Comparison................................................ 96

Figure A.1: GPS-INS Data/Vertical Gyro Comparison (Validation Set 1) ................... A-2

Figure A.2: Two State Roll and Pitch Error (Validation Set 1).................................. A-2

Figure A.3: GPS-INS Data/Vertical Gyro Comparison (Validation Set 2) ................... A-3

Figure A.4: Two State Roll and Pitch Error (Validation Set 2) ................................. A-3

Figure B.1: GPS-INS Data/Vertical Gyro Comparison (Validation Set 1) ................... B-2

Figure B.2: Method III Roll and Pitch Error (Validation Set 1) ................................. B-2

Figure B.3: GPS-INS Data/Vertical Gyro Comparison (Validation Set 2) ................... B-3

Figure B.4: Method III Roll and Pitch Error (Validation Set 2) ................................. B-3

Figure C.1: : GPS-INS Data/Vertical Gyro Comparison (Validation Set 1) ................. C-2

Figure C.2: Method IV Roll and Pitch Error (Validation Set 1) .................................. C-2

Figure C.3: GPS-INS Data/Vertical Gyro Comparison (Validation Set 2) ................... C-3

Figure C.4: Method IV Roll and Pitch Error (Validation Set 2)................................ C-3 


\section{Nomenclature}

\section{Symbol}

\section{English}

A

B

D

E

$\mathrm{H}$

$\mathrm{Hz}$

I

J

$\mathrm{J}$

$\mathrm{J}(\varphi, \theta, \psi)$

$\mathrm{K}$

N

$\mathrm{P}$

$P$

Q

$\mathrm{R}$

$\mathrm{R}$

$U$

V

$\mathrm{X}$

a

a

b

$\mathrm{cm}$

d

diag

$\mathrm{dt}$

f

e

g

$\mathrm{h}$

hot's

$\mathrm{k}$

$\mathrm{m}$

\section{Description}

System Matrix

Input Matrix

Feed-forward Matrix

Ephemeris Broadcast Errors

System Output/Observation Matrix

Hertz

Identity Matrix

Optimal Control Performance Index

Jacobian Matrix

Jacobian Matrix for Aircraft Attitude Angles

Kalman Gain

Line Normal to the Ellipse

Error Covariance Matrix

Position from the Equator to the Line Normal of the Ellipse

Model/Input Noise Covariance Matrix

Measurement Noise Covariance Matrix

Transformation Matrix

GPS x-axis Velocity

GPS y-axis Velocity

Matrix Containing Any Real Number

IMU Acceleration Values ( $\mathrm{x}, \mathrm{y}, \mathrm{z}$ axis)

Semi-major Axis Length

Semi-minor Axis Length

Centimeter

Lever Arm Correction Postion Offset Distance (x,y,z)

Diagonal Matrix

Time Increment

Ellipsoid Flatness

Ellipsoid Eccentricity

Gravitational Force

Altitude

Higher Order Terms

Discrete Time Increment

Meter 


\section{Greek}

$\Phi$

$\Phi$

$\alpha$

$\eta$

$\theta$

$\lambda$

$v$

$\rho$

$\sigma$

$\varphi$

$\chi$

$\psi$

$\omega$

$\omega$

\section{Subscript}

0

A

B

$\mathrm{e} 2 \mathrm{t}$

$\mathrm{p}$
Angular Roll Rate

Angular Pitch Rate

Angular Yaw Rate

Reference Acceleration for GPS Attitude Determination

Time

System Input Vector

GPS Velocity (x-axis)

GPS Velocity (y-axis)

GPS Velocity (x,y,z axis)

GPS Velocity (z-axis)

n-Dimensional State Vector

Projection Along the $\mathrm{x}$-axis

m-Dimensional State Vector

Projection Along the y-axis

Projection Along the z-axis

Measurement Vector

State Transition Matrix

Longitude

Alpha

Receiver Tracking Error Noise

Pitch Angle

Latitude

Noise Vector (Measurement Noise)

Pseudorange

Variance

Roll Angle

Clock, Multi-path, Ephemeral, and the Receiver Tracking Error

Heading Angle

Noise Vector (Model/Input Noise)

Angular Rate

Initial Position/Condition

Lever-Arm Point One

Lever-Arm Point Two

EFEC to Tangent Frame

Roll Rate 


\section{Acronym}

AGV

ANS

AoA

ARE

CAPM

CAT

$\mathrm{CG}$

CT

DARPA

DCM

DOF

ECEF

ECI

EEG

ENU

EKF

ERGM

FOG

GPS

IMU

KF

INS

LMS

LORAN

LRG

MSE

MAV

MEMS

MP

MIT
Pitch Rate

Yaw Rate

Tangent Frame to EFEC

GPS Unknown Receiver Coordinates

Projection Along the $\mathrm{x}$ Axis

Projection Along the $y$ Axis

Projection Along the $\mathrm{z}$ Axis

Autonomous Ground Vehicles

Autonomous Navigation System

Angle of Attack

Algebraic Riccati Equation

Capital Asset Pricing Model

Computed Axial Tomography

Center of Gravity

Computational Tomography

Defense Advanced Research Projects Agency

Direction Cosine Matrix

Degree-of-Freedom

Earth-Centered Earth-Fixed

Earth-Centered-Inertial

Electroencephalogram

East-North-Up

Extended Kalman Filter

Extended Range Guided Munitions

Fiber Optic Gyroscope

Global Positioning System

Inertial Measurement Unit

Kalman Filter

Inertial Navigation System

Least Mean Square

Long Range Navigation

Laser Ring Gyroscope

Mean Squared Error

Micro Air Vehicle

Micro-Electrical Mechanical Systems

Multi-path Error

Massachusetts Institute of Technology 


$\begin{array}{ll}\text { NASA } & \text { National Aeronautics and Space Administration } \\ \text { NED } & \text { North-East-Down } \\ \text { OBC } & \text { On-Board Computer } \\ \text { P/D } & \text { Price/Dividend } \\ \text { PSD } & \text { Power Spectral Density } \\ \text { R/C } & \text { Radio Controlled } \\ \text { RTK } & \text { Real Time Kinematic } \\ \text { RLS } & \text { Recursive Least Squares } \\ \text { RMSE } & \text { Root Mean Squared Error } \\ \text { S\&P } & \text { Standard and Poor's } \\ \text { SA } & \text { Selective Availability } \\ \text { SAIC } & \text { Science Applications International Corporation } \\ \text { STD } & \text { Standard Deviation } \\ \text { STM } & \text { State Transition Matrix } \\ \text { TLC } & \text { Time to Lane Crossing } \\ \text { UAV } & \text { Unmanned Aerial Vehicle } \\ \text { UTM } & \text { Universal Transverse Mercator } \\ \text { VG } & \text { Vertical Gyro } \\ \text { WVU } & \text { West Virginia University }\end{array}$




\section{Chapter 1. Introduction}

\subsection{Problem Definition}

Over the past twenty years, there has been an increase in demand for the development of unmanned aerial vehicles (UAV) and more recently, micro aerial vehicles (MAV), which has spurred research in a variety of areas within the aerospace industry. These areas include, but are not limited to: guidance and navigation (which is the main focus for this research objective), structures and materials, sensor design and development, propulsion systems, and communications.

This thesis focuses in the area of guidance and navigation, mainly the development of an aircraft navigation system utilizing a low-cost, off-the-shelf sensor package implemented on an YF-22 test-bed designed and constructed at West Virginia University (WVU).

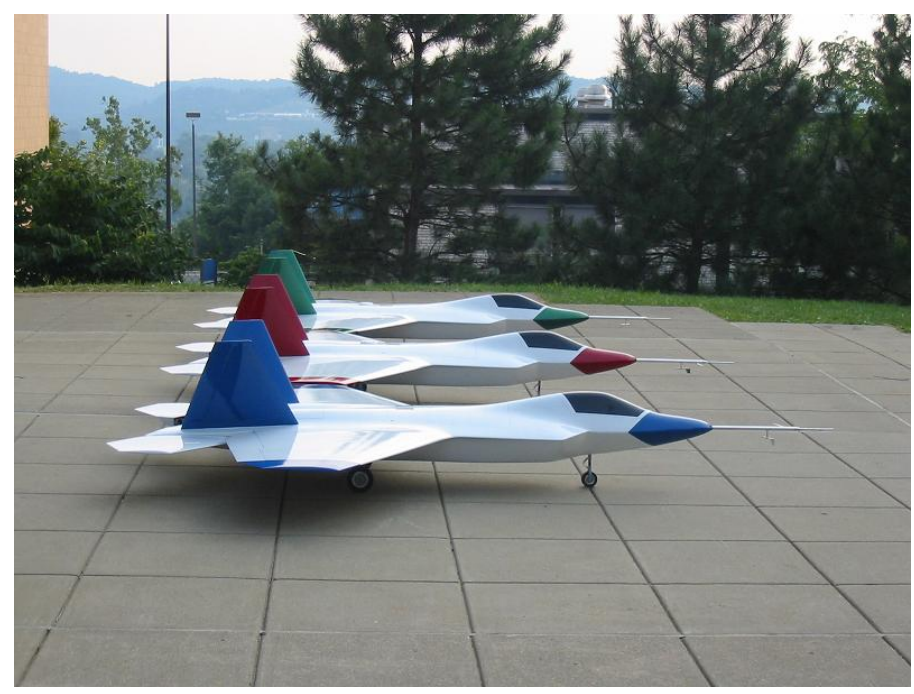

Figure 1.1: WVU YF-22 Research Test-beds

The test-beds were constructed for an Air Force research project in which WVU successfully achieved autonomous formation flight on three YF-22 test-beds. A radio control (R/C) pilot controlled a virtual 'leader' while two 'follower' aircraft flew in a triangle pattern autonomously ${ }^{1,2}$. The 'leader' aircraft transmitted position, velocity, and attitude information to the follower aircraft for use in the control algorithm. To achieve autonomous formation flight the three aircraft were equipped with a variety of sensors, which include an IMU, GPS, and Vertical Gyro for the aircraft Euler angles. 
The vehicles sensor package, in respect to the research conducted in this thesis, is composed of a low-cost inertial measurement unit (IMU), global positioning system (GPS), vertical gyroscope (VG), and flight computer.

The Vertical Gyro is a mechanical gimbaled component which determines aircraft attitude data at a high level of accuracy and high frequency, with the drawbacks of consuming a great deal of power, high cost, and has a shorter lifespan due to the ability of the mechanical parts to wear over time.

The IMU is capable of producing the attitude, position, and velocity with low power consumption while at a generally low cost. An IMU is composed of accelerometers and gyroscopes orthogonal to one another, which are integrated to obtain vehicle position, velocity and attitude, in which this integration is called an inertial navigation system (INS).

INS position, velocity and attitude are based solely on the previous measurement from the IMU's accelerometers and gyros. This makes the INS a self contained closed system, which has positive and negative aspects. The positive aspects are that the system doesn't rely on a reference point, which would limit the navigation system to a limited area.

The negative aspects of the system are that the sensors generate a great deal of noise such that the integration of this noise over time generates a so-called "drift" in the attitude causing the parameters to be inaccurate to the point where they are unusable for flight control. Since the system is self-contained without any outside correction, the error grows without bound which can be minimized with the use of either higher precision sensors (which come with a high price), or the use of various filtering methods. In the case of UAV and MAV design, parameter accuracy and precision in many cases is sacrificed for other variables such as sensor cost, weight, and availability, which are design criteria that must be taken into account when navigation and control systems are being implemented.

In addition, navigation systems comprised of higher precision components such as laser ring gyroscopes (LRG) and fiber optic gyroscopes (FOG), for example, are limited by the fact that the government regulates the sale and distribution of such components. These gyroscopes also cost in the range of US\$100,000, which also limit the use of the 
gyro in everyday consumer applications such as automobiles and general aviation. The majority of the applications for these types of components are generally restricted to military, government-sponsored research, NASA applications, and commercial airlines.

The final sensor utilized in this research project is GPS, which also has good and bad aspects associated with it. On the positive side, new GPS position and velocity data is obtained at each new time increment causing it to be unsusceptible to drifting effects, although GPS data can be degraded at each individual measurement by such caused error occurrences as satellite loss, atmospheric effects, multi-path effects, selective availability (SA), interference, jamming, and ephemeris and clock errors.

In regards to the previously described components, this thesis relates the use of sensor fusion to the application of vehicle navigation, for which the inertial measurement unit and global positioning system are fused to combine the complimentary aspects (closed system for IMU, while GPS is insusceptible to drift) between the GPS and IMU data while removing the negative attributes from one another.

Basically, Sensor fusion is the combination of sensory data from multiple sources in the attempt to improve data quality or generate data that could otherwise not be obtained from independent sensors. Data between the various sources must in some way complement one other, meaning the sensor's data must have some trait or link in common that allows for the fusion.

An example of sensor fusion is contrasting two sensors with two individuals. Let's say that the two individuals are working independently on a similar problem in two rooms' side-by-side in which they are both stuck on different calculations. Since they are working independently they may never determine the answer they are looking for, however by putting the two together they can combine their knowledge and help one another solve each of their calculations. This comparison can be reverted back to sensors in which each sensor may contain certain elements to aide one another.

The combination of the GPS data (which does not drift), with IMU data (which is not reliant on external measurements), is the same as combing the two individuals to collaborate on a similar problem. The IMU can benefit the GPS measurement by not be reliant of the external measurements while the GPS can benefit the IMU by not being susceptible to the drift, eliminating the negative effects from each measurement device 
leaving only the accurate data that is comparable to its higher precision, higher cost sensor counterparts. With the complimentary effects between the GPS and IMU data, a Kalman filter is a perfect fit to correct the drift issue, in which several methods are discussed to deal with such error.

The first method utilizes the IMU, GPS, and Vertical Gyro to correct the position and velocity only. This method shows how a Kalman filter is implemented so that the GPS and IMU complement each other; such that the IMU's position and velocity are corrected for drift errors while the GPS is corrected for the caused error described earlier.

The Vertical Gyro attitude is used in this method for simplicity; by using the IMU attitude values the system becomes nonlinear which drastically increases the complexity of the system. This issue is addressed in the second method described below.

The second method addressed eliminates the Vertical Gyro and uses a method of manipulating the GPS velocity data to obtain attitude information for use in the Kalman filter to correct the IMU's attitude. The IMU's attitude values are used creating the need for the extended Kalman filter (EKF) due to the nonlinear characteristics of the INS integrations. This not only increases the complexity of the calculations but also increases the amount of computational load on the computer posing concerns for use in real-time applications.

The third method for this section was then implemented utilizing the extended Kalman filter to estimate the error in the estimated position, velocity, and attitude. This method corrects the system states by utilizing only the GPS position and velocity as the measured values for use in the filter, without using the GPS estimated attitude. The states to be estimated in the filter are the error states of the position, velocity and attitude, instead of the actual dynamic system states estimated in the first method.

\subsection{Research Objectives}

The following research objectives are somewhat of a blueprint outlining the process in which the research requirements are met. They are intended to address the development and evaluation of the different sensor fusion methods discussed throughout this thesis. 
- Develop an INS system using the Matlab ${ }^{\circledR}$ programming environment using IMU data obtained during the WVU formation flight research project.

- Develop and test a data fusion algorithm using the Matlab ${ }^{\circledR}$ programming environment using GPS, Vertical Gyro, and INS data to improve position and velocity of a vehicle. This task utilizes Vertical Gyro data so that the nonlinear effects of the attitudes in the INS can be neglected allowing for the use of a Kalman filter. Validation data sets are simulated to compare the error analysis between the validation and initial development set.

- Develop and test data fusion software using the Matlab ${ }^{\circledR}$ programming environment using GPS and INS values to improve the position, velocity, and attitude of the vehicle. This task uses the INS attitude requiring the use of a nonlinear model, which in turn requires the use of the EKF. A method to determine the GPS attitude is used as a means for determining the residual within the EKF. Validation data sets are simulated to compare the error analysis between the validation and initial development set.

- Develop and test data fusion software using the Matlab ${ }^{\circledR}$ programming environment using GPS and INS values to improve the position, velocity, and attitude of the vehicle. This task uses the INS attitude requiring the use of a nonlinear model, which in turn requires the use of the EKF. The residual within the EKF for the attitude correction is determined through state error analysis. Validation data sets are simulated to compare the error analysis between the validation and initial development set.

\subsection{Thesis Overview}

The chapter structure throughout this thesis is organized in the following manner:

- Chapter 2 is composed of the literature review, which presents descriptions of the various researches being conducted within the field of sensor fusion. The majority of this section is composed of work conducted on navigation systems although additional sensor fusion applications are discussed.

- Chapter 3 is composed of all of the underlying theory that is the basis of this research topic. Navigation systems are very complex and involve many forms of 
higher-level mathematics involving geometry, trigonometry and calculus, while also requiring an understanding of aircraft flight dynamics. This chapter describes in detail the various coordinate frames and their corresponding transformations, INS development and integration, GPS ephemeral/pseudorange position calculations, GPS attitude determination, and the detailed discussions describing the underlying theory behind the Kalman filter and extended Kalman filter.

- The fourth chapter is devoted to the experimental setup of the research project, in which all of the theory from the previous two chapters is combined into a detailed description such that the final solution is obtained. The hardware setup, data acquisition for the laboratory experiments, and limitations are also discussed within the context of this chapter.

- Chapter 5 displays the results are presented in a manner so that the reader can see how and where sensor fusion is beneficial for low-cost navigation systems. This includes a detailed error analysis along with a computational workload analysis.

- Chapter 6 then concludes the thesis with the closing remarks, which contain conclusions drawn from this research effort, recommendations, and continuing efforts leading to actual vehicle implementation. 


\section{Chapter 2. Literature Review}

\subsection{General Description}

Sensor and data fusion is widely used and is on the forefront of navigation and autonomous control research. The ability to combine multiple data sources enables the capability of a dynamic system to not be restricted by individual measurements, but combines all of the information on hand to generate a better more refined measurement of the system parameters.

Although Kalman filtering is the only method of sensor fusion used throughout the research portion of this thesis, it would be unfair to not touch on the Weiner Filter, for it is the original basis behind the Kalman Filter.

The Wiener filter was developed by Norbert Wiener in the early years of World War II to design a controller for anti-aircraft guns that could "predict" where to shoot so that a round would hit enemy aircraft using noisy radar data. This was accomplished by minimizing the mean-square error between the output and the desired output ${ }^{3}$. This minimization of the error allowed estimation for the future position of the aircraft.

Unlike land and naval ballistics at the time, the speed of the aircraft was not a negligible parameter in the prediction algorithm; this resulted in the past trajectory of the aircraft to be used to extrapolate the future position. Wiener found that since the filter was based on probability and statistics, an exact predicted location could not be obtained, but only a better guess, similar to that of weather forecasting ${ }^{4}$.

Although the filter proved to be a highly effective in the prediction of aircraft trajectories, it was too complicated to be implemented by soldiers in the field. The research was not conducted in vain, for which Wiener's work in the field of communication theory, which led to the formulation of cybernetics. The theory behind the Weiner filter was also the basis behind the Kalman filter as described in the next section. 


\subsection{Kalman Filter Methods}

\subsubsection{Simple Kalman Filter}

Rudolf Emil Kalman originally determined the method in November 1958, when he thought of the idea to apply state variables to the Weiner filter. After increasing his knowledge on probability theory, Kalman equated expectation and projection to derive the Weiner filter into the Kalman filter ${ }^{5}$.

The filter is a recursive filter that estimates the states of a dynamic system by comparing the covariance of the state estimate with the covariance of a measurement at a certain time, $t$. This process is separated into two steps, the first being the state propagation using the system dynamic model with the inputs being noisy sensor measurements. These measurements corrupt the state estimates, introducing the second step, in which the Kalman filter is implemented to take advantage of the system dynamics to reduce the error, ultimately correcting the estimated state ${ }^{6}$. The Kalman filter, when implemented correctly is an optimal estimator in which the best possible, optimal, estimate of the states can be obtained.

While for most applications the filter is designed discretely, but can also be implemented in continuous time using the Kalman-Bucy Filter ${ }^{7}$. The main distinction between the discrete Kalman filter and the Kalman-Bucy filter, is that the measurement and update steps described above are not distinct. This is due to the fact that the update of the error covariance matrix is determined in a single calculation instead of independent a priori and a posteriori calculations. This single calculation can occur because the observation noise in the a priori calculation occurs at the same time as the a posteriori estimate.

When Kalman developed the simple Kalman filter, more commonly referred as simply, the 'Kalman Filter,' it was initially derived for linear systems, though it didn't take long for various renditions of the filter to expand it into non-linear form as seen in the following section. 


\subsubsection{Extended Kalman Filter}

The first credited application of the Kalman filter was on the Apollo Moon Program in which the filter was incorporated on the Apollo's navigation computer ${ }^{8}$. In 1959 NASA was in need of a system to navigate to the moon in which space flight navigation posed problems due to the fact that there was no nonmoving reference point to reference the flight path of the spacecraft. Stanley F. Schmidt came up with the idea of applying the Kalman filter to obtain guidance and navigation data. Schmidt successfully implemented the filter using the optical measurements of the stars and inertial measurements of the spacecraft with a level of precision high enough to insert the spacecraft in orbit around the moon. This application to guidance was a groundbreaking achievement in which the Kalman filter was then incorporated into all navigation systems.

This first implementation was named the Kalman-Schmidt filter, or more commonly called the extended Kalman filter, which proved that nonlinear systems can be implemented in the Kalman filter. The linearization of the dynamic system, measurement model, or both are generally conducted with the use of the Taylor series expansion in which the value at each time increment is an estimate of the nonlinear system at that time increment. Nonlinear estimation techniques are effective in which the time increment, $d t$, in-between estimates are relatively small. Additional nonlinear measurement methods can be seen in Ref [9] and [10].

Problems can arise with the EKF mainly due to the fact that, unlike the Kalman filter, it is not a true optimal estimator. With the filter no longer being optimal, the $a$ priori and a posteriori covariance matrices are no longer true covariance matrices. In other words, a correct system model and proper values for the initial state and error covariance matrices are essential so that the filter does not diverge building from the errors generated by the linearization.

\subsubsection{Unscented Kalman Filter}

In 1997 researchers Simon J. Julier and Jeffery K. Uhlman at Oxford published a new linear estimator in which a set of discretely sampled points were used to parameterize mean and covariance. These sampled points were selected by a sampling 
technique known as the unscented transform, in which points were selected around the mean, where they are then propagated through the non-linear function to determine the covariance of the estimate. This method removes the step using Jacobians to linearize the dynamic model while obtaining more accurate values for the true mean and covariance of the system ${ }^{11}$.

The thought behind the unscented Kalman filter is to approximate the mean and covariance distribution, unlike the EKF, which approximates the system models. Julier and Jeffrey's methodology was to leave the system models intact since they are more precise than the estimated values for the mean and covariance and has proven to be an effective filter in many applications a few of which can be seen in the next section.

\subsection{Sensor Fusion Applications}

Sensor fusion applications have been applied to many fields of research including aerospace, ground robotics, naval, munitions, agricultural, economics and the medical fields, while research into more consumer and medical applications is driven as sensor technology advances and cost decreases. Sensor technology over the past ten years alone has seen a drastic reduction in size and cost allowing for the creation of more “intelligent," affordable consumer products.

The majority of the applications in this literature review are based around guidance and navigation; however additional applications are discussed that show how sensor fusion has been utilized in different situations. There are still additional applications that exist which are not covered within the context of this literature review.

\subsubsection{Naval Applications}

Naval research in guidance and navigation during the early parts of the twentieth century can be accredited with development of the first navigation systems in which $\mathrm{E}$. A. Sperry developed the first gyrocompass for use within large steel ships ${ }^{12}$. This first gyrocompass was installed in August of 1911 aboard the U.S.S. Delaware which paved the way for Sperry to apply his vast knowledge of gyroscopes to produce an array of products including the first full gun battery system, which was installed on all battleships 
during World War I, a gyro stabilization system which kept the vessel from rolling, and the first gyro pilot steering mechanism dubbed the nickname "Metal Mike."

One hundred years later, naval research is still on the forefront of autonomous navigation research. As with many areas within the military, there is a large amount of research effort being put forth into the creation of autonomous, or semiautonomous vehicles, in which the U.S. navy is in the process of producing three such vehicles; the $\mathrm{DD}(\mathrm{X})$ destroyer, $\mathrm{CG}(\mathrm{X})$ cruiser, and the LCS littoral combat ship ${ }^{13}$.

In conglomeration with the Navy is an array of companies involved in the development of the new technologies needed to create such a navy of the future. These vehicles have implemented sensor fusion techniques throughout the vessels so that sensor packages can generate more precise data while also providing crew members with a wider range of data so that decisions could be based on multiple angles.

Lockheed Martin has developed a series of simulations in relation to the $\mathrm{DD}(\mathrm{X})$ in which tests were arranged to track aircraft, ships, submarines, and land targets under various warfare scenarios. Measures of the sensor fusion performance were evaluated across multiple scenarios which incorporated five different sensors, in which Lockheed claims that their sensor fusion technology is the only one that is capable of processing all of the sensor inputs at the level of precision needed in real time ${ }^{14}$.

An additional goal the Navy is pushing for is to reduce the manning requirements to approximately one-third of what is required on the ships of today in which Northrop Grumman is conducting research in this area ${ }^{15}$. In order to reduce the manning by such a magnitude requires the use of data fusion and intelligent agents that analyze data such as a human would. This technology would require the onboard computer to make decisions by collecting and analyzing data across a magnitude of sensors which then creates multiple courses of action along with recommendations to the available crew members so that the manning could be reduced while also minimizing human error.

Northrop Grumman is also working on the development of an autonomous system to discover undersea threats to the new naval vehicles ${ }^{16}$. The use of sensor fusion within the detection of undersea threats allows for acoustic sensors to be integrated with nonacoustic sensors to further enhance the precision and localization of undersea threat detection. 


\subsubsection{Aerospace Applications}

E.A. Sperry's developments in the field of controls also influenced the early days of the aeronautical industry during the second decade of the twentieth century in which Lawrence Sperry, the son of E.A. Sperry, applied a lightweight adaptation of his father's gyroscope to a Curtiss C-2 Biplane which he coupled with the control surfaces to maintain strait and level flight ${ }^{17}$. This mechanism implemented on the C-2 was the first autopilot integrated on an aircraft, which was first demonstrated in Paris in 1913. L. Sperry's inventions also include the artificial horizon, improved anemometer, and the horizontal and vertical gyro that allowed for the development of the autopilot. This initial autopilot has been refined and improved over the years with the development of improved inertial sensors, GPS, and improved control theory. Sperry has also been credited with being the founder of the mile-high club.

Since then, sensor fusion has made its way into every aspect of the aerospace industry; ranging from guidance and navigation, ground target detection, to noise cancellation, and so on. In the area of guidance and navigations alone, the addition of sensor fusion methods have allowed for a great reduction in cost, size, weight, and power consumption which in turn generates the need for a flight computer that can handle the additional computational load. In many cases the additional benefits in data precision obtained by incorporating Kalman filtering techniques by far out-weigh the additional computation resources required as discussed in the following paragraphs.

Researchers at the Munich University of Technology have developed a series of algorithms to provide general aircraft pilots with information about the aircraft angle-ofattack (AoA), sideslip, and wind information along with highly accurate navigation information. The purpose is to utilize low-cost commercial off-the-shelf components along with no major modifications to the aircraft to produce the flow around the aircraft, wind information, and navigation parameters. The wind vector was determined analytically using attitude, velocity, and position data from the INS/GPS system along with pitot tube air speed. The aircraft speed vector was then differenced by the air speed to establish the wind vector and the AoA and sideslip angles were obtained with the use of the INS/GPS, control surface deflections, and aircraft aerodynamic model data ${ }^{18}$. 
In a more elaborate example of the advancements in navigation, control, and sensor fusion; Boeing has been developing a rotorcraft that is capable of being fully autonomous from takeoff to landing. In July of 2006, the rotorcraft named Unmanned Little Bird took off, hovered, and then flew a programmed intelligence, surveillance, and reconnaissance mission before returning to land ${ }^{19}$. The development of such a vehicle is of great value during times when the pilot is needed to complete additional tasks or has become incapacitated due to unforeseen circumstances.

The autonomous take-off and landing of the rotorcraft alone proved to be a task in itself to overcome due to the high level of guidance and navigation control needed to achieve the task, especially when attempting a shipboard landing. A shipboard landing increases the complexity of the task by adding wind over deck and wake turbulence, which creates challenging and unpredictable conditions during take-off and landing. This topic has been researched in conglomeration between Boeing and NovAtel to design a navigation system capable of providing the level of precision needed to maintain control in such environments ${ }^{20}$. The system acts similar to that of determining a GPS receiver's position in terms of pseudorange and ephemeral data such that the helicopter is the GPS receiver and two separate points onboard the ship acts as the satellite positions. The points onboard the ship are known from actual GPS real time kinematic (RTK) data which is then used to determine the relative position to the helicopter using a "pseudorange" vector. The helicopter's position is known from and onboard GPS/INS navigation system, which is used with the "pseudorange" vector to determine the relative distance to the point of landing.

An additional example of the integration of micro components into aerospace applications is being investigated by researchers at the University of Florida in conjunction with the NASA Langley Research Center is the autonomous flight and control of Micro Air Vehicles (MAV) equipped with only small video cameras and transmitters $^{21}$. Their goal is to successfully navigate the MAV using a forward facing camera to determine the aircraft attitude with the use of horizon detection algorithms. In order for the attitudes to be implemented in a control scheme, they must first be filtered so that the high frequency noise and single frame errors are removed. The Kalman filter is appealing for this application due to its ability to remove the previously stated errors 
without having the benefit of an accurate dynamic model. The flex-wing MAV (Figure 2.1) is modeled as two first-order, constant velocity systems due to the fact that no dynamic model is available for the system.

The MAV itself does no actual data processing, in which all of the data is transmitted to a ground station, from there it is processed and the necessary servo control is transmitted back to the MAV.

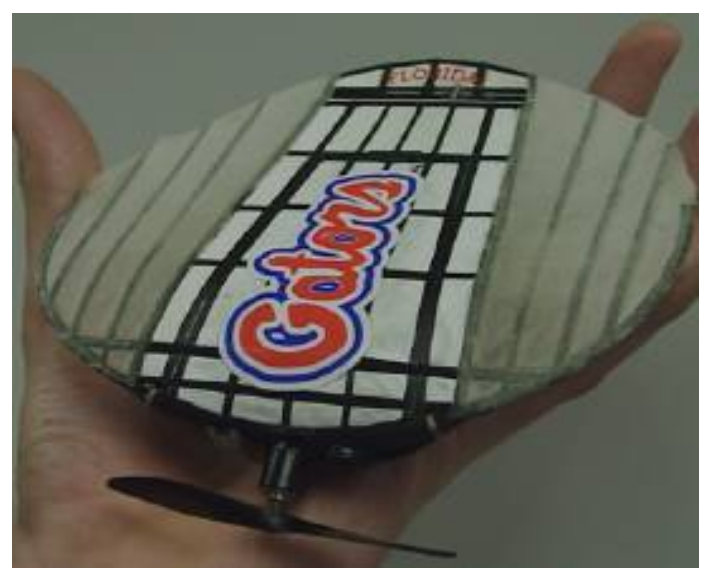

Figure 2.1: 6" Flex Wing MAV

A further example in machine vision navigation, researchers at West Virginia University have researched the integration of GPS/machine vision navigation using the extended Kalman filter for use in the area of aerial refueling ${ }^{22}$. The extended Kalman filter is used to combine the position data from a GPS/machine vision based system for providing a reliable estimation of the relative position of the UAV in regards to the tanker position. Machine vision is used in this effort to compliment the GPS during times of signal loss or degradation, which in the case of aerial refueling, can be attributed to the tanker airframe impeding satellite line of sight.

As previously discussed, the initial implementation of the Kalman filter was applied for space navigation issues during the Apollo program. From the time of Schmidt's first application of the EKF on the Apollo mission, to the navigation system used on the space shuttle's orbiter, many changes and advances in technology have improved the way navigation is conducted in space. One area greatly influenced by sensor fusion methods is the development and integration of sensors incorporated into satellites position and control algorithms. The size of satellites has also decreased drastically due to the low cost and ability to construct and launch in a reasonably short 
duration. As a consequence of the reduction in size, there has also been a reduction in computation power, sensors and actuators. These smaller, cheaper sensors are less precise, generating the need for research in satellite position determination and navigation. Due to the lack of GPS data, additional sensors such as sun sensors, star trackers, and magnetometers are integrated with IMU calculations to correct for INS drift as seen in reference [23].

Noise reduction and cancellation has also become a topic drawing a great deal of interest in the aerospace industry. In the past few years an increasing number of commercial headphones have been incorporating noise-canceling filters to remove unwanted noise from the surrounding environment. These civilian devices mainly use least mean square (LMS) and recursive least square (RMS) filtering techniques which produce acceptable results, although Kalman filtering methods have been tested and produce better results than the previously listed methods, however it tends to generate a level of computational load that is too high for application in the civilian sector ${ }^{24}$. For example, researchers at Massachusetts Institute of Technology ${ }^{25}$ (MIT) have been investigating the reduction of helicopter, propeller aircraft, and jet aircraft noise using a single microphone in which a Kalman filter was implemented to aide in the noise reduction. These systems have been implemented in various military aircraft producing excellent results, with the exception of a hefty price tag.

\subsubsection{Ground Vehicle Applications}

With sensor technology advancing and cost decreasing, fusion techniques are being extensively used in ground vehicles with a wide range of applications. A great deal of research effort is being put forth into autonomous navigation systems (ANS) on autonomous ground vehicles (AGV). This type of research is being conducted on all makes, models and sizes of vehicles to conduct an array of tasks.

The first application discussed is the highly publicized 2005 Defense Advanced Research Projects Agency (DARPA) challenge. This event is a 132-mile race in which research teams were to design a fully autonomous vehicle to navigate its way through an off-road terrain course in which the winner was awarded two million dollars. Of the 23 
teams that competed in the race, Stanford completed the race first in a time of 6 hours, 53 minutes, 8 seconds ${ }^{26}$.

The Stanford's team utilized sensor fusion for localization of the vehicle Euler angles and position in the Universal Transverse Mercator (UTM) coordinate system. An extended Kalman filter was used to asynchronously integrate data from the GPS and IMU at a maximum rate of $100 \mathrm{~Hz}$. The vehicle's onboard computer then geo-references the EKF position data with data obtained from two laser range finders, radar, and vision data so that the most efficient path can be taken.

Sensor fusion applications also include the incorporation of data fusion into civilian vehicles, in which many of the vehicles on the road today already have the necessary sensors available to implement some form of sensor fusion. Speed sensors, electronic compasses, GPS navigation systems, rear proximity sensors, and electric power steering are just a few sensors widely used on many vehicles produced today. While some car manufacturers such as Toyota that have integrated such components as throttle by wire and electric brake force distribution, and the Lexus LS460L, which has actuators for steering, braking, and throttle, are used for parallel parking. All of these components can and are in some cases integrated into sensor fusion algorithms to increase safety and vehicle performance.

One application in which standard vehicle components are being utilized is from researchers at the University of Michigan, in which they have conducted research on road departure warning systems using a Kalman filter to estimate the lateral velocity and the heading angle so that the Time to Lane Crossing (TLC) value can be determined ${ }^{27}$. The benefit of knowing the TLC is due to the fact that the majority of vehicle road departure accidents in the US are associated with a single vehicle departing the roadway due to loss of control or inattentiveness. With this TLC the university's goal is to develop a system to warn drivers when they are drifting inadvertently off the road. The available measurements are; the lane position obtained from an advanced camera system that measures lateral displacement, steering angle, lateral acceleration, yaw rate, and forward velocity using the vehicles speed sensor. The Kalman filter in this application is to filter the measured data, estimate the lateral velocity, and provide measurement values when 
sensors become temporarily unavailable. With these values, the TLC parameter can easily be determined.

\subsubsection{Munitions}

An additional industry pushing for the development of smaller, affordable, accurate sensors are the munitions sector. The development of precision-guided munitions (PGM) is not only pushing for sensor cost and size reduction, but must also be able to function under high-g environments over $15,000 \mathrm{~g}^{\text {' }} \mathrm{s}^{28}$.

The Navy's Extended Range Guided Munitions (ERGM) and the Army's Excalibur programs are two of the driving force behind munitions research. The ERGM research program effort began in 1994 in conjunction with Raytheon winning the contract to develop a munition that that had autonomous capabilities in which could be fired from existing firing mechanisms with little modification.

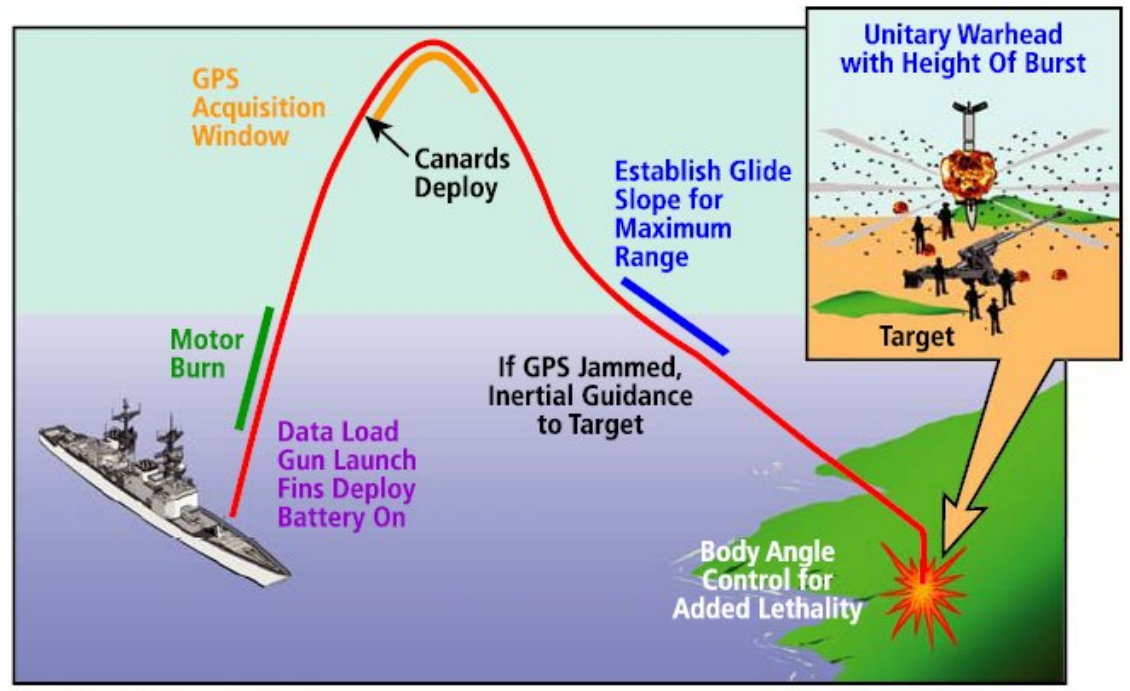

Figure 2.2: Flight Trajectory of EX-171 Munition $^{29}$

Raytheon developed the EX-171 rocket-assisted 5" projectile, which is a 12calibur projectile, capable of carrying a 4-calibur sub-munition. The munition is equipped with a coupled INS/GPS guidance system, which allows for accurate guidance during points of GPS loss and jamming in environments with electronic countermeasures $^{29}$. Since the start of the program, numerous flight tests have been conducted with fairly successful results with the first test being in February 2001, Figure 2.2 presents a rendering of a EX-171 flight trajectory. 
The ERGM has had many setbacks due to the need to develop new technology. MIT's Lincoln Laboratory was brought in to conduct an independent assessment and determined that the research being conducted was beneficial and that for the amount of new technology being developed there was substantial progress being made ${ }^{29}$.

One of the major breakthroughs due to the ERGM program was the advancement in technology on the level of inertial navigation. BAE systems was subcontracted to develop the SiIMU02 IMU that could meet the sensitivity requirements of precision guided munitions, packaged in enclosures that could withstand 20,000 g's, significantly reduced the cost, and reduced the calibration time from eight days for one IMU to four IMU's in three days ${ }^{30}$.

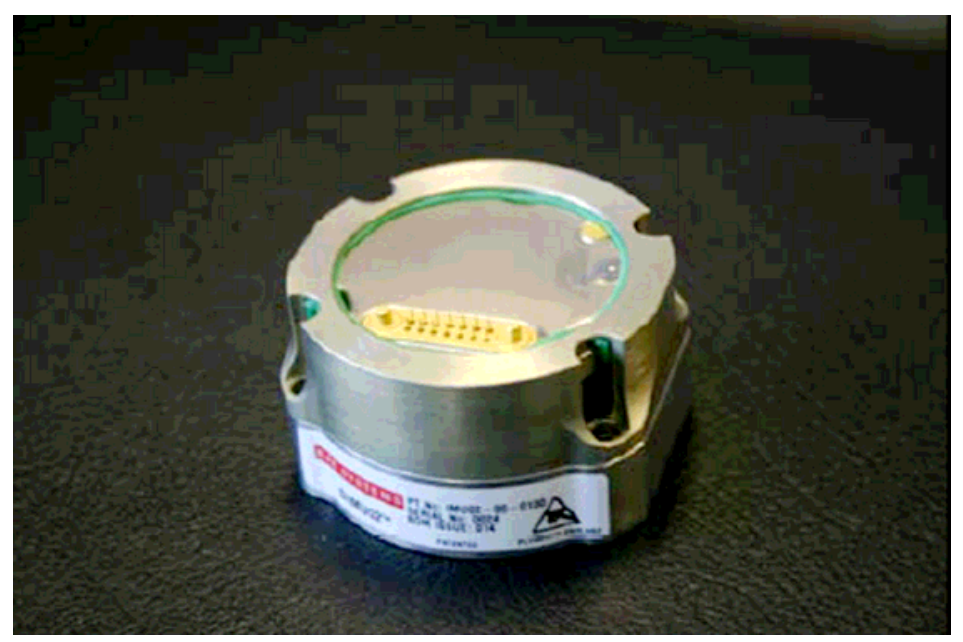

Figure 2.3: SiIMU02 IMU Developed by BAE Systems for ERGM Research

The Army's XM982 Excalibur $^{31}$ is a $155 \mathrm{~mm}$ precision-guided extended range artillery projectile. The munition is fire and forget which is canard controlled with a GPS/INS guidance system. The munitions purpose is to utilize existing and future 155 $\mathrm{mm}$ howitzer platforms to produce a weapon that has a range and accuracy greater than that of current ballistics.

In the case that the GPS is jammed the INS will be used as the primary guidance system to the target. In the situation where initial GPS data cannot be established, the munition will follow the fired ballistic trajectory with no aided guidance. Due to this feature the munition must be fired with accuracy within $35 \mathrm{~m}$ for area targets and less than $10 \mathrm{~m}$ for targets requiring a direct hit. 


\subsubsection{Medical Industry}

Sensor fusion methods have also proven beneficial in the medical industry in such areas as medical imaging, prosthetic limb and organ development, neural prosthesis, and epilepsy diagnosis. As was true with all other fields discussed, just a few medical applications are touched on, although there are infinite possibilities in which sensor fusion methods can be implemented in the medical field.

Within the field of medical imagery researchers at the University of Hawaii have been testing photon laser applications to produce computational tomography (CT) scaned images, also known as computed axial tomography (CAT) scans ${ }^{32}$. The benefits of using the photon laser over the standard X-ray is that the photon laser does not require healthy tissue cells to be exposed to the strong radiation beam used to detect the unusual tissue cells. The unusual tissue is detected by knowing the scattering and absorption coefficients of the both the healthy and unhealthy tissue, in which this shows a distinction between the tissues. The regeneration of the image is difficult due to the calculation of the photon diffusion equation, in which the solving the forward and inverse problem creates issues. The forward problem is defined as having the cells' scattering and absorption coefficients allowing the computation of the photon density within the medium. Since all of the values within the forward problem are can be determined, this is not where sensor fusion applications are beneficial, leading to the inverse problem. In the inverse problem, detectors measure the photon density, which is used to reconstruct the tissue structure by estimating the scattering and absorption coefficients. The solving of the inverse problem for this application is set up as a parameter identification problem, in which parameters of healthy tissue are established as an initial baseline. The initial "healthy" values are then compared to the noisy values read by the detectors in the EKF to converge on the actual parameters of the tissue with anomalies.

In the area of neural prosthesis, a multitude of research has been conducted in attempts to collect neural signals utilizing implanted electrodes for use in the control of prosthetic limbs or computer cursors ${ }^{33,34,35}$. In one series of tests, electrodes were chronically implanted into macaque monkey's arms to collect the neural signals during a series of computer tests requiring the monkey to "play" two simple video games ${ }^{33}$. A model was created to mimic the hand kinematics in which the collected neural signals 
would be used to mimic the monkey's responses to the games. Kalman filtering was used to decode the neural data, which allows for the filtering of the non-Gaussian distributions of cell firing rates. In addition the filter helps to clean each individual electrodes signal in cases when multiple cell firings are detected simultaneously.

An electroencephalogram (EEG) is the main tool in the diagnosis of epilepsy in which Kalman filtering can be a valuable tool in the detection of epileptic spikes ${ }^{36}$. Normally EEG data is read visually by an experienced EEG technician which can be time consuming and difficult due to varying brain activity which could represent epileptic spikes which could be interpreted as false positives or negatives by a human eye. By incorporating a KF to review the data, then ran through a thresholding function, EEG data can be reviewed unsupervised minimizing human error while also reducing technician reviewing time.

\subsubsection{Economics}

The production, distribution, and consumption of goods and services is complicated to model due to its unpredictable nature; meaning there's no finite model to predict the economies exact 'dynamics.' There are simply too many variables that cannot be accounted for such as natural disasters, wars, and disease, for example, however, there are signs and trends that allow for educated guesses to help determine which direction the market is heading, which is a perfect fit for Kalman filtering applications.

Economists Lorne Johnson and Georgios Sakoulis have conducted research on a method of implementing a Kalman filter that estimates time varying sensitivities to predetermined risk factors to determine which financial sector has the highest risk and growth potential ${ }^{37}$. The purpose was to find a successor that could outperform the Capital Asset Pricing Model (CAPM), the standard model in use today, for which their model accounts for the change in dividend yield on the S\&P 500 composite index, change in the spread between the ten year treasury note and the 90 day treasury bill yield, percent change in the near month crude oil contract, and the change in the default spread. Simulations ran over various sample periods show that the model does at least as well as the CAPM at pricing risk, though the method produces better results during periods of high economic uncertainty and business cycle turning points such as the period following 
the equity market peak in 2000. It is however; less effective during periods (e.g. 19942000) when stock price/dividend (P/D) ratios are higher which indicate higher returns in the future which make it difficult to quantify, and cannot be easily adapted into the macroeconomic model developed in this article.

Another application for Kalman filtering in the area of economics is for economic forecasting, which is the process where predictions are made about various or all variables within an economy ${ }^{38}$. For example, in agriculture forecasting, the determination of the amount of food needed is an important issue that can lead to higher costs, supply shortages, or overproduction as discussed in [39] at Shandong Institute of Mining and Technology in Jinan, China, where a Kalman filter was implemented on an Bayesian dynamic linear model for forecasting pork production. 


\section{Chapter 3. Theoretical Background}

\subsection{Overview of Theoretical Approach}

The theoretical approach to this research can be broken into three stages. The first stage involves the coordinate frame descriptions and their respective transformation calculations between reference frames. These are reviewed in detail throughout Section 3.2 since there is a great deal of interaction between data in multiple reference frames. Section 0 then describes the INS calculation process including the respective drift error involved in the integration. The fourth section is dedicated to the determination of GPS attitude estimation. All of which are measurement values used in the Kalman filter and EKF. Finally the Kalman filter and EKF process and calculations are discussed in Section 3.5.

\subsection{Coordinate Frames of Reference}

As discussed above, this section reviews and compares different reference frames and the relationship between one another. This section has been broken into two sections; Section 3.2.1 discusses each coordinate frame while Section 3.2.2 describes how to relate each coordinate system to one another. The methods and transformations presented in this section discussed in greater detail in $[40,41,42]$.

\subsubsection{Coordinate Frame Descriptions}

The understanding of navigation systems is heavily dependant on the underlying knowledge of each individual coordinate frame. This section is devoted to an in-depth discussion of each navigation system in a manner such that the reader understands the terminology used throughout this project.

\subsubsection{Inertial Frame}

An inertial frame is a frame of reference that is fixed about an arbitrary point that is not affected by rotational effects, but can still be in a constant motion. For example the Earth-centered-inertial (ECI) coordinate system that defines coordinates on earth that is non-rotating with the $\mathrm{x}$-axis pointing toward the vernal equinox (an imaginary vector that 
originates at the center of the earth, through the equator and points directly to the sun). The vernal equinox is the point in time when the equatorial plane and the sun align; this occurs the first day of spring and the first day of fall. Figure 3.1 shows a graphical representation of the ECI coordinate frame.

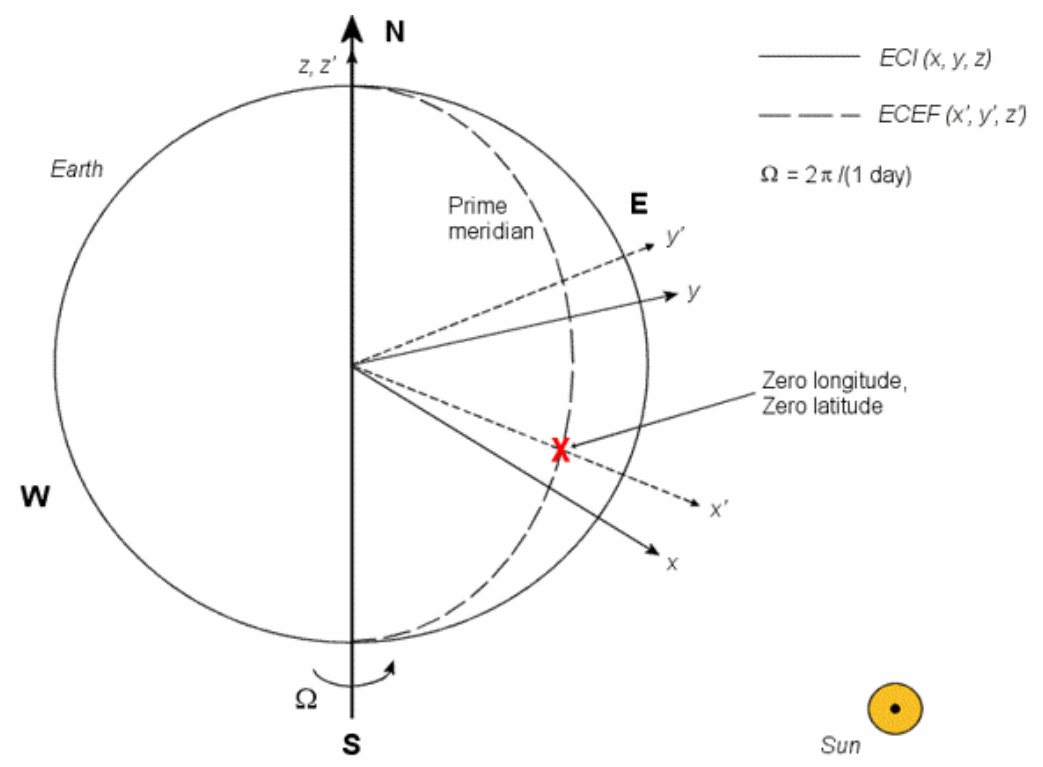

Figure 3.1: ECI and ECEF Coordinate Frame Representation ${ }^{43}$

The inertial frame allows positions to be defined on a local level user-defined initial position. In the majority of cases, the positions and trajectories needed aren't affected by the rotation of the earth allowing this to be neglected, although long duration position tracking, such as transatlantic flights and long-range ballistic missiles must take earths rotational effects into account.

\subsubsection{Earth-Centered Earth-Fixed (ECEF)}

The Earth-Centered Earth-Fixed Coordinate system is set in relation to the earth, meaning that the location given in an ECEF coordinate system rotates with the earth. There are two general coordinate system conventions for the ECEF reference frame; rectangular coordinates and geodetic coordinates, which are discussed in greater detail below. 


\subsection{Earth-Centered Earth-Fixed (ECEF) Rectangular Coordinates}

The ECEF rectangular coordinates depict a position in relation to Cartesian (x,y,z) coordinates with the $(0,0,0)$ location being the center of the earth. The $\mathrm{x}$ component propagates through 0 degrees longitude (prime meridian or Greenwich meridian) and 0 degrees latitude (equator), and the $y$-axis is perpendicular to the $\mathrm{x}$-axis on the equator. The $\mathrm{z}$ component points upward through the North Pole. Figure 3.1 demonstrates a representation of the ECEF rectangular coordinate system, note that as the earth rotates the coordinate system rotates in unison while the ECI stays fixed.

\subsubsection{2. $\quad$ Earth-Centered Earth-Fixed (ECEF) Geodetic Coordinates}

The ECEF geodetic coordinate system is expressed in latitude $(\lambda)$, longitude $(\Phi)$, and height $(h)$ and is the primary method for depicting position for many applications, such as navigation, surveying, and GPS. The geodetic system stems from the fact the earth is not round but an ellipse, which causes the need for an ellipsoidal model.

Over the years different ellipses to define earth's shape have been developed which created an error between coordinate positions due to ellipse size deviation. The World Geodetic System 84 (WGS84) has been accepted as the primary ellipsoid parameters for the majority of the world, which are listed in Table 3-1 ${ }^{44}$.

Table 3-1: WGS84 Parameters

\begin{tabular}{|c|c|}
\hline Semi-major Axis Length, a (m) & $6,378,137.0$ \\
\hline Semi-minor Axis Length, $\mathrm{b}(\mathrm{m})$ & $6,356,752.3$ \\
\hline Ellipsoid Flatness, $f$ & $\frac{a-b}{a}=0.0034$ \\
\hline Ellipsoid Eccentricity, $e$ & $\sqrt{f(2-f)}=0.0818$ \\
\hline
\end{tabular}

As seen in Figure 3.2 the latitude $(\lambda)$ is the angle that shows the position of $P$ from the equator to the line normal of the ellipse (earth's surface). The line normal to the ellipse $(N)$ extends from the surface of the ellipse to the intersection of the z-axis. The longitude $(\Phi)$ is the angle from the prime meridian to the longitudinal plane where point 
$P$ intersects with the equator, and the altitude $(h)$ is the distance from the surface of the ellipse to point $P$.

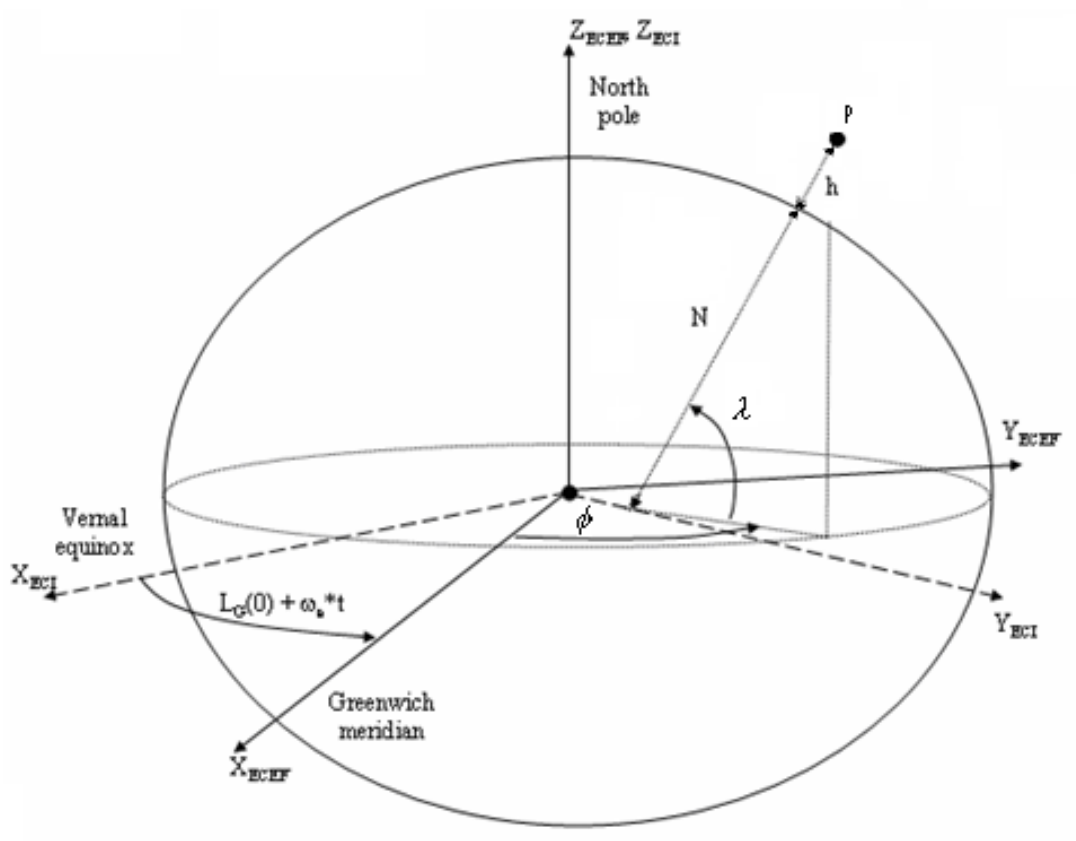

Figure 3.2: ECEF Geodetic Frame

\subsubsection{Tangent Plane}

The tangent plane, or navigation frame, is an inertial frame of reference that is localized. It is a frame of reference that depicts a position by placing a plane tangent to the earth's surface at the specific point of reference. The point of reference can be any arbitrary point at which location points can be referenced. An example is in the case of an aircraft and a radar station. The radar station would be the point of reference of the tangent plane and the aircraft's position would be referenced from that point.

The tangent frame is divided into two separate conventions; East, North and Up (ENU) or the North, East and Down (NED) convention. The ENU axes are placed orthogonal to each other with the $\mathrm{x}$-axis pointing East on the tangent plane, the $\mathrm{y}$-axis pointing North on the plane, and the z-axis pointing up perpendicular to the plane. The NED axes are placed orthogonal to each other with the $\mathrm{x}$-axis pointing north on the tangent plane, the y-axis pointing east on the plane, and the z-axis pointing down 
perpendicular to the plane. This project only refers to the NED convention; ENU will not be discussed past this section.

\subsubsection{Body Frame}

A body frame is a coordinate frame of reference directly connected to a vehicle. The three axis of the body frame are orthogonal and generally placed at the center of gravity of the vehicle, with the $y$-axis perpendicular to the $\mathrm{x}$-axis following the right-hand rule, and the z-axis perpendicular to the plane generated by the $\mathrm{x}$ and $\mathrm{y}$-axis. Since the body frame is directly connected to the vehicle, there is no way of telling where it is at a given time. Therefore an additional stationary coordinate frame is needed, such as a tangent or ECEF reference frame, to act as a reference axis so that the vehicles movement through space can be tracked. Figure 3.3 shows the body frame in relation to the ECEF coordinate frame.

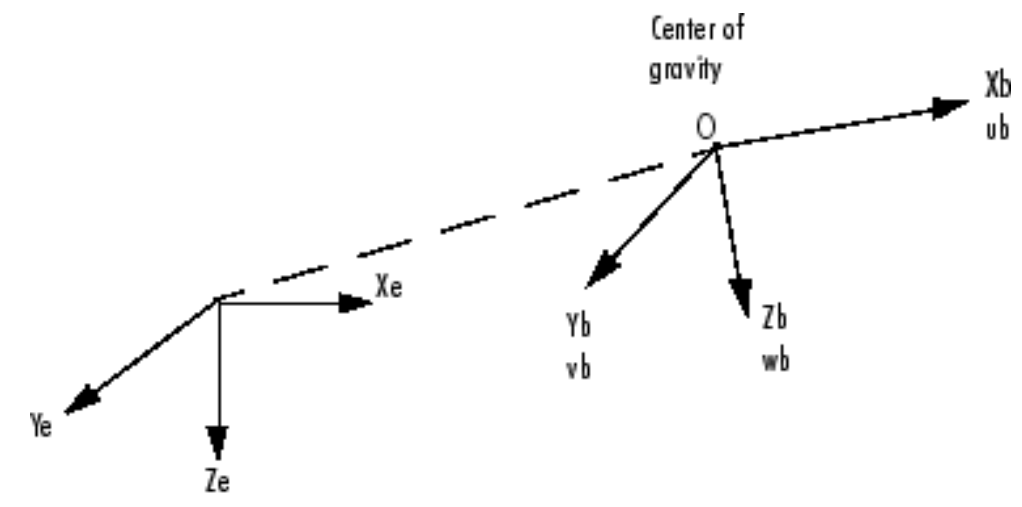

Earth-fixed reference frame

Figure 3.3: Body Frame in Relation to ECEF ${ }^{43}$

\subsubsection{Platform Frame}

The platform frame is located on the vehicle, generally at an offset from the body frame, with each respective axis parallel to that of the body frame. The purpose of the platform frame is to compensate for a sensor that is located on the body of the vehicle, but not on the center of gravity. The platform frame can be the same as the body frame when the sensor is located on or near the same axis of the body frame. 


\subsubsection{Reference Frame Conversions}

Since there are many different frames of reference used, a means of converting between each reference frame is needed as a way of comparing and relating data together. This section reviews the transformations used throughout this thesis.

\subsubsection{ECEF Geodetic to ECEF Rectangular}

The conversion from geodetic to rectangular coordinates is a simple calculation in which the WGS-84 ellipsoid parameters listed in Table 3-1 are used to determine the normal vector leading to the rectangular coordinates conversion shown below ${ }^{40}$ :

$$
\begin{gathered}
N=\frac{a}{\sqrt{1-e^{2} \sin (\lambda)^{2}}} \\
x=(N+h) \cdot \cos (\lambda) \cdot \cos (\phi) \\
y=(N+h) \cdot \cos (\lambda) \cdot \sin (\phi) \\
z=\left[N \cdot\left(1-e^{2}\right)+h\right] \cdot \sin (\lambda)
\end{gathered}
$$

where $N$ is the length of the normal vector from the surface of the ellipse to the intersection of the $\mathrm{z}$ axis, $e$ is the eccentricity of the ellipse, $\lambda$ is the latitude coordinate position in ECEF geodetic coordinates, $h$ is the altitude above the surface of ellipse and $\Phi$ is the longitudinal position in the ECEF geodetic coordinate system.

\subsubsection{ECEF Rectangular to ECEF Geodetic}

There are several methods to convert rectangular to geodetic coordinates, all of which are more complicated than the conversion from ECEF geodetic to rectangular. Only one method is discussed within the context of this paper, although additional iterative methods can be seen in [40]. The method described in Table 3-2 below is accurate up to meter level precision and is non-iterative ${ }^{45}$. The reason for using the noniterative method is due to the fact that the iterative method produces a level of accuracy to the centimeter level, which is not necessary since the hardware utilized in this project is only capable of attaining meter level accuracy. 
Table 3-2: ECEF Rectangular to Geodetic Coordinate Conversion ${ }^{45}$

$\begin{aligned} p & =\sqrt{x^{2}+y^{2}} \\ E^{2} & =a^{2}-b^{2} \\ F & =54 \cdot b^{2} \cdot z^{2} \\ G & =p^{2}+\left(1-e^{2}\right) \cdot z^{2}-e^{2} \cdot E^{2} \\ c & =\frac{e^{4} \cdot F \cdot p^{2}}{G^{3}} \\ S & =\left(1+c+\sqrt{c^{2}+c \cdot c}\right)^{1 / 3} \\ P & =\frac{F}{3 \cdot\left(s+\frac{1}{s}+1\right)^{2} \cdot G^{2}} \\ Q & =\sqrt{1+2 \cdot e^{4} \cdot P} \\ r_{0} & =-\frac{P \cdot e^{2} \cdot p}{1+Q}+\sqrt{0.5 \cdot a \cdot(1+1 / Q)-\frac{P \cdot\left(1-e^{2}\right) \cdot z^{2}}{Q \cdot(1+Q)}-0.5 \cdot P \cdot p^{2}} \\ U & =\sqrt{\left(p-e^{2} \cdot r_{0}\right)^{2}+z^{2}} \\ V & =\sqrt{\left(p-e^{2} \cdot r_{0}\right)^{2}+\left(1-e^{2}\right) z^{2}} \\ z_{0} & =\frac{b^{2} \cdot z}{a \cdot V} \\ e^{\prime} & =\frac{a}{b} e \\ \lambda & =\tan { }^{-1}\left(\frac{z+e^{12} \cdot z_{0}}{p}\right) \\ \phi & =a \tan 2(y / x) \\ h & =U \cdot\left(1-\frac{b^{2}}{a \cdot V}\right) \\ & \\ & \\ & \end{aligned}$

\subsubsection{ECEF to Tangent Plane}

The next transformation converts ECEF coordinates to a tangent frame of reference. A local tangent frame reference point $\left(x_{0}, y_{0}, z_{0}\right)_{e}$ must first be selected to act as the point of reference for all additional points $(x, y, z)_{e}$ thereafter. The ECEF rectangular and geodetic coordinates at this point must be known so that the new points can be related back to the reference point.

The geodetic coordinates for the reference point must be known so that a transformation matrix can be constructed to create a relationship between the ECEF and the tangent ENU convention. The transformation Matrix $\left(R_{e 2 t}\right)$ from the EFEC to tangent 
frame is made up of two rotations; the first rotation is to align the y-axis in the east direction about the $\mathrm{z}$ axis, and the second is to align the $\mathrm{z}$ axis about the new $\mathrm{y}$ axis so that it points directly down perpendicular to the $x-y$ plane.

\begin{tabular}{|l|l|l|}
\hline & $R_{\phi}=\left[\begin{array}{ccc|}\cos (\phi) & \sin (\phi) & 0 \\
-\sin (\phi) & \cos (\phi) & 0 \\
0 & 0 & 1\end{array}\right]$
\end{tabular} \mid

The transformation matrix then becomes:

$$
R_{e 2 t}=R_{\lambda} \cdot R_{\phi}=\left[\begin{array}{ccc}
-\sin (\lambda) \cos (\phi) & -\sin (\lambda) \sin (\phi) & \cos (\lambda) \\
-\sin (\phi) & \cos (\phi) & 0 \\
-\cos (\lambda) \cos (\phi) & -\cos (\lambda) \sin (\phi) & -\sin (\lambda)
\end{array}\right]
$$

where $\lambda$ and $\Phi$ is the latitude and longitude of the reference point.

To express the position of a random location in the tangent plane that is in ECEF coordinates, the tangent plane reference point $\left(x_{0}, y_{0}, z_{0}\right)_{e}$ must be subtracted from the location of interest $(x, y, z)_{e}$. This is calculated as follows:

$$
\left[\begin{array}{c}
x \\
y \\
z
\end{array}\right]^{\text {tangent }}=\mathrm{R}_{e 2 t} \cdot\left[\begin{array}{c}
x-x_{0} \\
y-y_{0} \\
z-z_{0}
\end{array}\right]^{E C E F}
$$

The transformation from the NED tangent frame to the ECEF frame can be reversed with the inverse of the transformation matrix. Due to the orthogonal properties of the matrix the inverse is also the transpose as follows:

$$
\left[\begin{array}{c}
x \\
y \\
z
\end{array}\right]^{E C E F}=\mathrm{R}_{e 2 t}^{T} \cdot\left[\begin{array}{l}
x \\
y \\
z
\end{array}\right]^{\text {tangent }}+\left[\begin{array}{c}
x_{0} \\
y_{0} \\
z_{0}
\end{array}\right]^{E C E F}
$$




\subsubsection{Tangent to Body Frame}

The transformation from the body axis to the navigation axis is performed using a transformation matrix, further described as the Direct Cosine Matrix (DCM). The DCM is calculated as follows, which transforms the navigation axis to the body axis:

$$
\begin{gathered}
D C M=\left[\begin{array}{ccc}
1 & 0 & 0 \\
0 & \cos (\phi) & \sin (\phi) \\
0 & -\sin (\phi) & \cos (\phi)
\end{array}\right]\left[\begin{array}{ccc}
\cos (\theta) & 0 & \sin (\theta) \\
0 & 1 & 0 \\
-\sin (\theta) & 0 & \cos (\theta)
\end{array}\right]\left[\begin{array}{ccc}
\cos (\varphi) & \sin (\varphi) & 0 \\
-\sin (\varphi) & \cos (\varphi) & 0 \\
0 & 0 & 1
\end{array}\right] \\
=\left[\begin{array}{ccc}
\mathrm{c}(\varphi) \cdot \mathrm{c}(\theta) & \mathrm{s}(\varphi) \cdot \mathrm{c}(\theta) & -\mathrm{s}(\theta) \\
-\mathrm{s}(\varphi) \cdot \mathrm{c}(\phi)+\mathrm{c}(\varphi) \cdot \mathrm{s}(\theta) \cdot \mathrm{s}(\phi) & \mathrm{c}(\varphi) \cdot \mathrm{c}(\phi)+\mathrm{s}(\varphi) \cdot \mathrm{s}(\theta) \cdot \mathrm{s}(\phi) & \mathrm{c}(\theta) \cdot \mathrm{s}(\phi) \\
\mathrm{s}(\varphi) \cdot \mathrm{s}(\phi)+\mathrm{c}(\varphi) \cdot \mathrm{s}(\theta) \cdot \mathrm{c}(\phi) & -\mathrm{c}(\varphi) \cdot \mathrm{s}(\phi)+\mathrm{s}(\varphi) \cdot \mathrm{s}(\theta) \cdot \mathrm{c}(\phi) & \mathrm{c}(\theta) \cdot \mathrm{c}(\phi)
\end{array}\right]
\end{gathered}
$$

The transformation from the tangent plane to the body axis is then completed as follows:

$$
\left[\begin{array}{l}
a_{x} \\
a_{y} \\
a_{z}
\end{array}\right]^{\text {Body }}=D C M \cdot\left[\begin{array}{l}
a_{x} \\
a_{y} \\
a_{z}
\end{array}\right]^{\text {Tangent }}
$$

The transformation from the body axis to the navigation axis can be reversed with the inverse of the DCM. Due to the orthogonal properties of the matrix the inverse is also the transpose as follows:

$$
\left[\begin{array}{l}
a_{x} \\
a_{y} \\
a_{z}
\end{array}\right]^{\text {tangent }}=D C M^{T} \cdot\left[\begin{array}{l}
a_{x} \\
a_{y} \\
a_{z}
\end{array}\right]^{\text {bod } y}
$$

If the reader is unfamiliar with the DCM, there are many variations that can cause confusion and should refer to [46] or [47].

\subsection{Inertial Navigation System (INS)}

\subsubsection{INS Description}

An inertial navigation system (INS) is a form of dead reckoning and is system of measurements and integrations of differential equations to determine the position, velocity, and attitude of a vehicle. INS's are self-contained navigation systems that are not reliant on additional measurement devices other than their own measurements. This characteristic of INS's poses benefits as well as drawbacks to the output data. 
Having an INS onboard a vehicle allows the computation of the position, velocity and orientation without the limitation of the requirement of a having a point of reference. This is useful on vehicles such as submarines, aircraft, spacecraft, and ships travel long distances outside of the range of a given reference point. INS systems are also beneficial to for their inability to be jammed and are stealthy in nature. The system neither emits nor receives any detectable radiation and has no external antenna, which could be detectable by radar.

The drawbacks come from the fact that INS has no additional outside reference point. Each value for position, velocity and attitude is determined from the previous value through integration, therefore causing any error in the measurements to accumulate over time. This accumulation of error causes a drift from the actual value that grows exponentially. The amount of error compounded over time is reliant on the quality of the inertial sensors used for the INS measurements. Methods for correcting this error are discussed in later chapters.

The primary measuring device in an INS is an inertial measurement unit (IMU), which generally consists of accelerometers and rate gyros in orthogonal axes, and in some cases magnetometers. There are generally two types of IMU's: mechanizedplatform or gimbaled and strap-down systems, which are discussed in greater detail below.

\subsubsection{Mechanized-Platform}

A Mechanized-platform is an IMU that is based on a gimbaled mechanism. For instance, a three axis gimbaled platform (Figure 3.4) has three rings positioned on bearings orthogonal to one another. This allows the platform in the middle of the gimbal to remain unchanged no matter the angle in which the vehicle is positioned. To cancel the gyroscopic precession, two gyroscopes are placed at right angles to one another, spinning at the same velocity. 


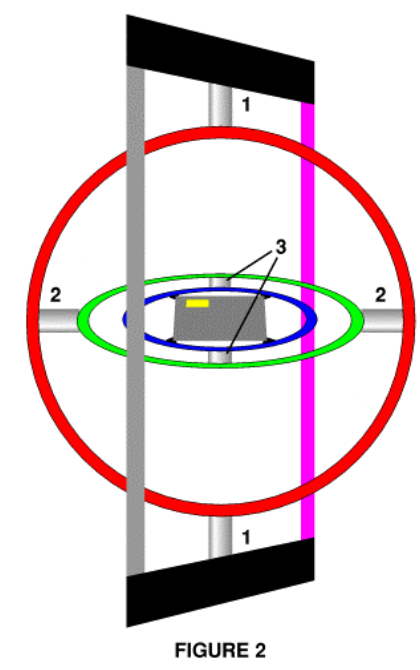

Figure 3.4: Gimbaled Platform ${ }^{48}$

The attitude of the vehicle can then be determined with the use of potentiometers at the location of the bearings, while the position and velocity can be determined by placing accelerometers on the gimbaled platform orthogonal to one another. Since the platform remains unchanged by the vehicle rotation, no coordinate transformation is required, as the gimbaled platform is consistent to the navigation axis.

Mechanized-platform systems produce results at a high level of accuracy with the drawbacks of being heavy, expensive, high in power consumption, and by having moving parts allows the component to wear over time.

\subsubsection{Strap-down Mechanization}

Strap-down IMU's are mounted directly to the inertial body and generally have three accelerometers and three gyros orthogonal to one another. Unlike the gimbaled IMU, a strap-down system calculates the vehicle's attitude with the use of a computer system based on the angular rates generated by the gyroscope. Then with the component attached directly to the vehicle, the accelerometer information is collected in the body axis, generating the need for a coordinate transformation so that the position and velocity of the vehicle can be determined along the navigation axis.

With the use of micro-electro-mechanical-system (MEMS) devices, strap-down systems eliminate all mechanical aspects allowing the IMU to be made much smaller, cheaper, weigh considerably less, and consume much less power than that of gimbaled systems, although the strap-down system is much less accurate than the gimbaled system 
while also requiring some sort of computer to integrate the accelerations and angular rates to position, velocity, and attitude data. The amount of additional computational load on a system could be a determining factor whether a strap-down or mechanized platform is preferred.

\subsubsection{INS Computation}

As stated previously a MEMS IMU's output of the angular rate and acceleration is integrated to determine position, velocity, and attitude estimates. Figure 3.5 shows a block diagram depiction of the computational layout of an INS system.

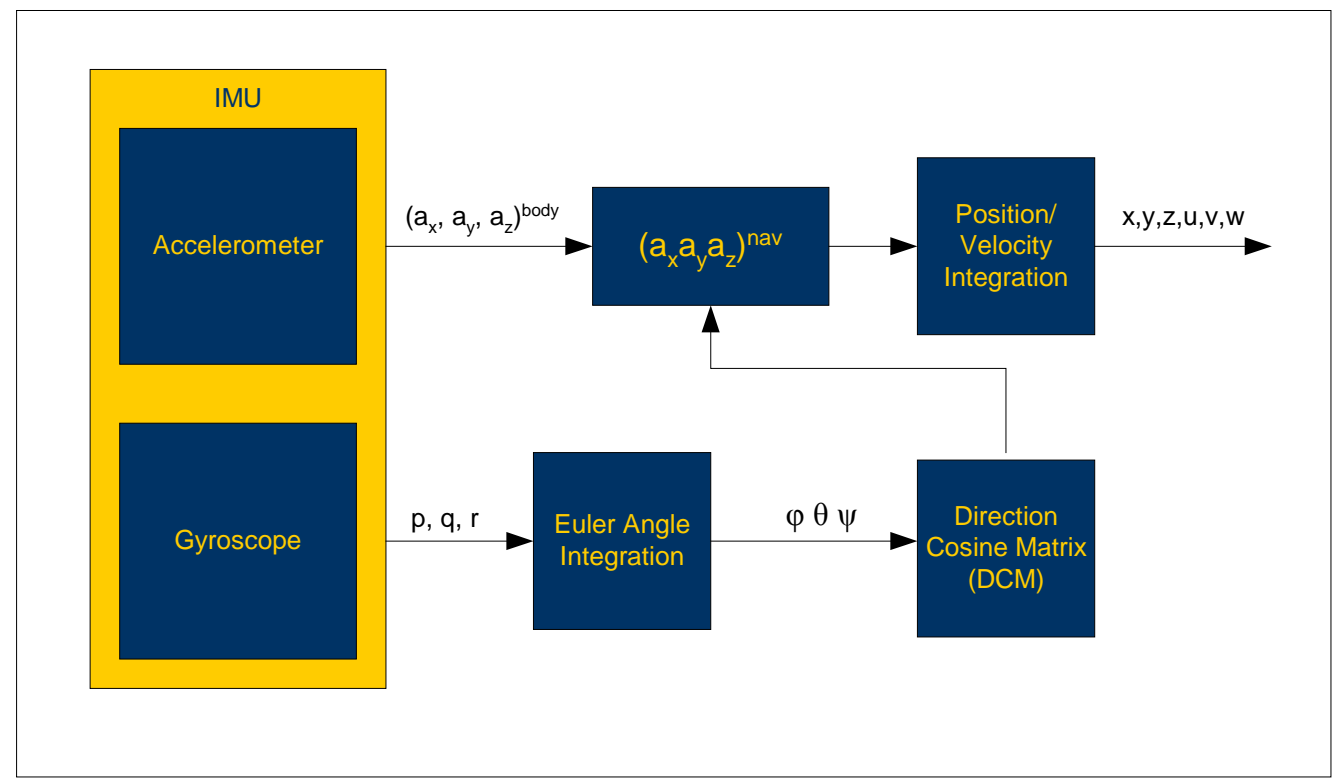

Figure 3.5: INS Computation

The calculation of an INS is composed of both linear and nonlinear components, which creates an issue in the implementation of a Kalman filter. This draws the need for a creation of a linearized model for the use in the filter, which is described later in this section. Keep in mind that the linearization of the nonlinear equations is used only in the filter itself, and that the actual state propagation estimate is calculated with the nonlinear equations.

The INS is modeled in state space for use in the Kalman filter that requires the linearization described in the previous paragraph. For the case of an INS the determination of the Euler angles and rotation matrices are the non-linear calculations in which a generalized discrete state space representation is as follows: 


$$
\begin{aligned}
& \dot{\mathrm{x}}_{\mathrm{k}}=\mathrm{A}_{\mathrm{k}} \cdot x_{k}+B_{k} \cdot u_{k}+w_{k} \\
& y_{k}=C_{k} \cdot x_{k}+D_{k} \cdot u_{k}+v_{k}
\end{aligned}
$$

where $A$ is the system matrix, $B$ is the input matrix, $C$ is the output matrix, $x$ is the ndimensional state vector, $y$ is the m-dimensional measurement vector, $w$ is the noise vector associated model/input error, $v$ is the noise vector associated with the measurement noise, $\mathrm{u}$ is the input vector, and $D$ is the feed-forward matrix.

The first step in the determination of the INS is to calculate the aircraft attitude values using the continuous aircraft kinematic differential equations as follows:

$$
\begin{gathered}
\dot{\phi}=\mathrm{p}+\mathrm{q} \cdot \sin (\phi) \cdot \tan (\theta)+\mathrm{r} \cdot \cos (\phi) \cdot \tan (\theta) \\
\dot{\theta}=\mathrm{q} \cdot \cos (\phi)-\mathrm{r} \cdot \sin (\phi) \\
\dot{\psi}=(q \cdot \sin (\phi)+\mathrm{r} \cdot \cos (\phi)) \cdot \sec (\theta)
\end{gathered}
$$

where $p, q$, and $r$ are the angular rates and $\phi$ and $\theta$ are the roll and pitch Euler angles.

As seen from the aircraft kinematic equations, the relationships are nonlinear, which creates the need for linearization of equations (3-15)-(3-17), which is accomplished with the use of a Taylor series expansion shown in equation (3-18) ${ }^{49}$.

$$
\left[\begin{array}{c}
\dot{\phi} \\
\dot{\theta} \\
\dot{\varphi}
\end{array}\right]=f(\phi, \theta, \psi, p, q, r)+\frac{\partial(\phi, \theta, \psi, p, q, r)}{\partial(\phi, \theta, \psi)}\left[\begin{array}{c}
\Delta \phi \\
\Delta \theta \\
\Delta \varphi
\end{array}\right]+\frac{\partial(\phi, \theta, \psi, p, q, r)}{\partial(p, q, r)}\left[\begin{array}{c}
\Delta p \\
\Delta q \\
\Delta r
\end{array}\right]+h^{\prime} t^{\prime} s
$$

where hot's are the "higher Order Term's" associated with the Taylor series expansion.

The system and input matrix from equation (3-18) is a Jacobian matrix of partial derivatives that generates the best linear approximation for a nonlinear system at each time increment, which allows for the state space approximation. The higher order terms error contribution generated from the Taylor series, which are neglected, could be reduced with the use of iteration, although for this research topic, the error will be reduced with the use of the Kalman filter discussed in the next chapter. Only the system matrix Jacobian is shown below although the input matrix is determined similarly. 


$$
\begin{gathered}
J(\phi, \theta, \psi)=\frac{\partial(\phi, \theta, \psi, p, q, r)}{\partial(\phi, \theta, \psi)}=\left[\begin{array}{ccc}
\frac{\delta f(\dot{\phi})}{\delta \phi} & \frac{\delta f(\dot{\phi})}{\delta \theta} & \frac{\delta f(\dot{\varphi})}{\delta \psi} \\
\frac{\delta f(\dot{\theta})}{\delta \phi} & \frac{\delta f(\dot{\theta})}{\delta \theta} & \frac{\delta f(\dot{\theta})}{\delta \psi} \\
\frac{\delta f(\dot{\psi})}{\delta \phi} & \frac{\delta f(\dot{\psi})}{\delta \theta} & \frac{\delta f(\dot{\psi})}{\delta \psi}
\end{array}\right]= \\
{\left[\begin{array}{ccc}
\mathrm{q} \cdot \cos (\phi) \cdot \tan (\theta)-\mathrm{r} \cdot \sin (\phi) \cdot \tan (\theta) & q \cdot \sin (\phi) \cdot \sec ^{2}(\theta)+\mathrm{r} \cdot \cos (\phi) \cdot \sec ^{2}(\theta) & 0 \\
-q \cdot \sin (\phi)-\mathrm{r} \cdot \cos (\phi) & 0 & 0 \\
(q \cdot \cos (\phi)-\mathrm{r} \cdot \sin (\phi)) \cdot \sec (\theta) & (q \cdot \sin (\phi)+\mathrm{r} \cdot \cos (\phi)) \cdot \sec (\theta) \cdot \tan (\theta) & 0
\end{array}\right]}
\end{gathered}
$$

Given that the angular rates and accelerations are measured at a certain time interval, $d t$, the linearized aircraft kinematic differential equations listed above must be converted to difference equations, or discrete time. The discretized nonlinear kinematic equations are listed below:

$$
\begin{gathered}
\phi_{k+1}=\phi_{k}+\left(\mathrm{p}_{k}+\mathrm{q}_{k} \cdot \sin \left(\phi_{k}\right) \cdot \tan \left(\theta_{k}\right)+\mathrm{r}_{k} \cdot \cos \left(\phi_{k}\right) \cdot \tan \left(\theta_{k}\right)\right) \cdot d t \\
\theta_{k+1}=\theta_{k}+\left(\mathrm{q}_{k} \cdot \cos _{k}\left(\phi_{k}\right)-\mathrm{r}_{k} \cdot \sin \left(\phi_{k}\right)\right) \cdot d t \\
\psi_{k+1}=\psi_{k+1}+\left(\left(q_{k} \cdot \sin \left(\phi_{k}\right)+r_{k} \cdot \cos \left(\phi_{k}\right)\right) \cdot \sec \left(\theta_{k}\right)\right) \cdot d t
\end{gathered}
$$

In which the discretized linear approximation of the Euler angles system matrix is then conducted as follows:

$$
\begin{gathered}
J_{\phi(\mathrm{k}+1)}=\phi_{\mathrm{k}}+\phi(k, \phi(k)) \cdot d t=\phi_{\mathrm{k}}+J(\phi, \theta, \psi) \cdot d t= \\
{\left[\begin{array}{ccc}
1+(\mathrm{q} \cdot \mathrm{c}(\phi) \cdot \mathrm{t}(\theta)-\mathrm{r} \cdot \mathrm{s}(\phi) \cdot \mathrm{t}(\theta)) \cdot d t & \left(q \cdot \mathrm{s}(\phi) \cdot \mathrm{sec}^{2}(\theta)+\mathrm{r} \cdot \mathrm{c}(\phi) \cdot \sec ^{2}(\theta)\right) \cdot d t & 0 \\
(-q \cdot \mathrm{s}(\phi)-\mathrm{r} \cdot \mathrm{c}(\phi)) \cdot d t & 1 & 0 \\
((q \cdot \mathrm{c}(\phi)-\mathrm{r} \cdot \mathrm{s}(\phi)) \cdot \mathrm{sec}(\theta)) \cdot d t & ((q \cdot \mathrm{s}(\phi)+\mathrm{r} \cdot \mathrm{c}(\phi)) \cdot \sec (\theta) \cdot \mathrm{t}(\theta)) \cdot d t & 1
\end{array}\right]}
\end{gathered}
$$

The discretized nonlinear kinematic equations (equations (3-20)-(3-22)) are then computed over time to obtain the INS Euler angle values. The linear approximation of the Euler angles will be revisited in Chapter 4 during the implementation of the extended Kalman filter. Figure 3.6 and Figure 3.7 show the IMU's angles compared to the vertical gyros output. It can be seen on the magnified plots that the IMU data carries the same trends as the vertical gyro although drift causes the increased magnitude of the peaks. 

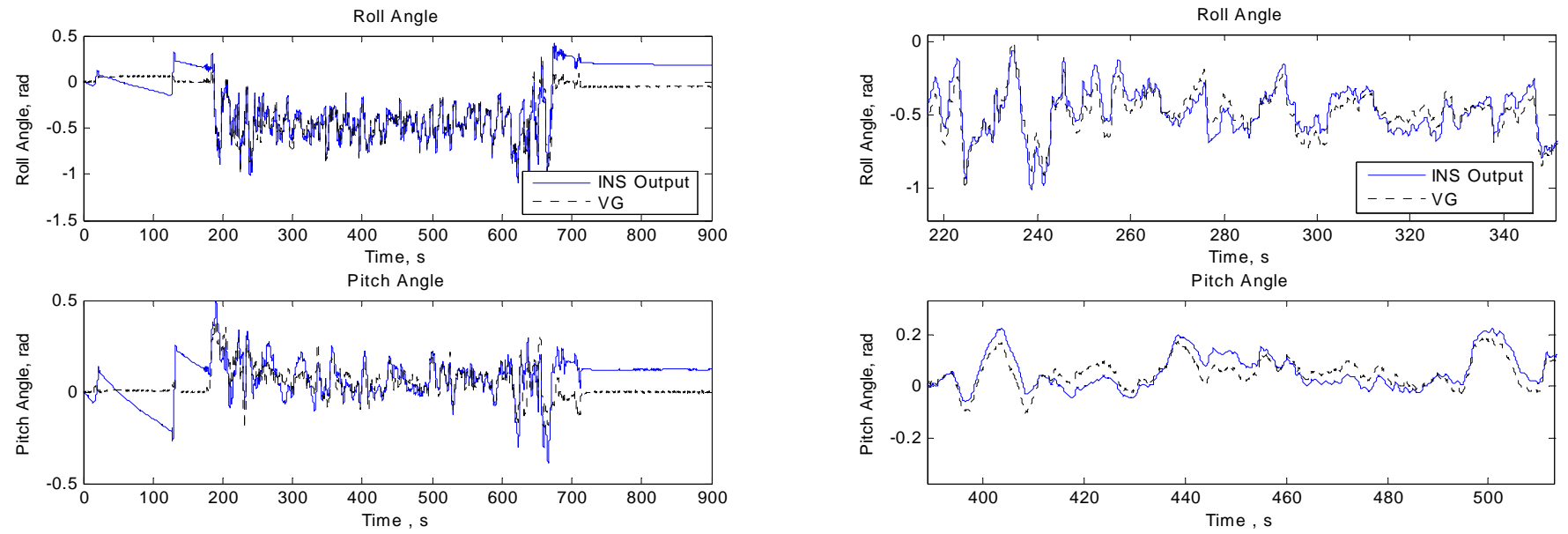

Figure 3.6. INS Data vs. Vertical Gyro Data

Figure 3.7. INS Data vs. Vertical Gyro Data (Magnified)

With the Euler angles determined, the focus now shifts to the IMU accelerometer data. The accelerations are first transformed from the body axis to the navigation axis using the DCM discussed earlier so that the integrated velocity and position can be directly related to the GPS data. The acceleration transformation is completed as follows:

$$
a_{3 x 1}^{n a v}=\left[\begin{array}{l}
a_{x} \\
a_{y} \\
a_{z}
\end{array}\right]^{\text {nav }}=D C M^{T} \cdot\left[\begin{array}{l}
a_{x} \\
a_{y} \\
a_{z}
\end{array}\right]^{\text {body }}
$$

where $a$ is the IMU acceleration values.

The position and velocity can then be easily calculated using the following discrete state space relationship:

$$
\left[\begin{array}{c}
x_{3 x 1, k+1} \\
v_{3 x 1, k+1}
\end{array}\right]=\left[\begin{array}{cc}
I_{3 x 3} & I_{3 \times 3} \cdot d t \\
0_{3 \times 3} & I_{3 \times 3}
\end{array}\right] \cdot\left[\begin{array}{c}
x_{3 x 1, k} \\
v_{3 x 1, k}
\end{array}\right]+\left[\begin{array}{c}
0_{3 x 3} \\
I_{3 \times 3} \cdot d t
\end{array}\right] \cdot\left[a_{3 x 1}^{n a v}\right]-\left[\begin{array}{c}
0_{5 x 1} \\
g
\end{array}\right]
$$

where $x$ is the position, $v$ is the velocity, and $\mathrm{g}$ is gravity.

By incorporating the Euler angle calculations into the position and velocity calculations the complete INS state space representation is:

$$
\left[\begin{array}{l}
x_{k+1} \\
v_{k+1} \\
\phi_{k+1}
\end{array}\right]=\left[\begin{array}{ccc}
I_{3 \times 3} & I_{3 x 3} \cdot d t & 0_{3 \times 3} \\
0_{3 \times 3} & I_{3 \times 3} & 0_{3 \times 3} \\
0_{3 \times 3} & 0_{3 \times 3} & J_{\phi(k+1)}
\end{array}\right] \cdot\left[\begin{array}{l}
x_{3 x 1, k} \\
v_{3 x 1, k} \\
\phi_{3 x 1, k}
\end{array}\right]+\left[\begin{array}{cc}
0_{3 \times 3} & 0_{3 \times 3} \\
I_{3 \times 3} \cdot d t & 0_{3 \times 3} \\
0_{3 \times 3} & J_{\phi(k+1)} \cdot d t
\end{array}\right] \cdot\left[\begin{array}{c}
a_{3 x 1}^{n a v} \\
\omega_{3 x 1}
\end{array}\right]-\left[\begin{array}{c}
0_{5 x 1} \\
g \\
0_{3 \times 1}
\end{array}\right]
$$

where $\omega$ is the aircraft angular rates and $g$ is gravity 
Keep in mind that Equation (3-26) is a linearized approximation and that additional error is introduced here since the hot's from the Taylor series approximation have been neglected. The state space model of the INS system with the linearized kinematic equations is for use only in the extended Kalman filter, which is discussed in detail in Chapter 4 and Chapter 5, otherwise using the nonlinear equations would produce better results since there is no error due to the hot's.

Figure 3.8 compares the components of the velocity about each axis. These values are overlaid and compared with the GPS flight data values, in which the compounding drift error is clearly visible.
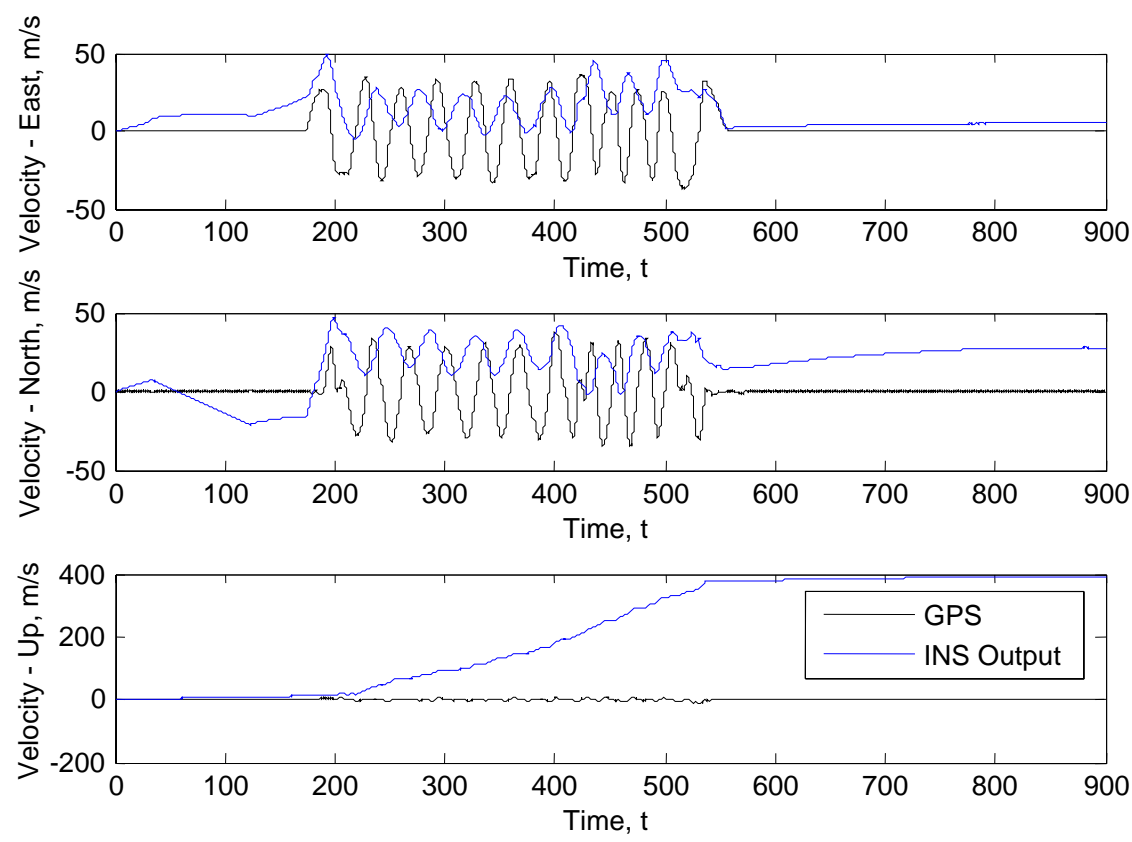

Figure 3.8: INS Data vs GPS Comparison

Note, the position information was not displayed due to its lack of relevance. After the position integration, there was little resemblance to the GPS position data; therefore this plot was intentionally neglected.

\subsection{GPS Calculations}

The creation of GPS, officially named NAVSTAR by the U.S. Dept. of Defense, has revolutionized the field of navigation and control in its ability to obtain precise position and velocity at nearly all points on the globe. Prior to the development of GPS various additional navigation methods (post World War II era), in which GPS was partly 
based from, were used to obtain accurate position data such as Long Range Navigation (LORAN) and the Decca Navigator, although these methods are being phased out due to the popularity and effectiveness of GPS. The following section describes the determination of GPS attitude that will be used in the next chapter to aide the filtering process.

\subsubsection{GPS Attitude Estimation}

This section describes the method used to determine vehicle attitudes with the use of the GPS velocity and IMU's accelerometer data. This method for estimating the aircraft attitude generates accurate but extremely noisy values that are useful as the measurement values for the Kalman filter that is discussed in the next chapter ${ }^{50}$. The GPS heading angle $(\varphi)$ was first determined by utilizing the GPS velocity on the ' $X$ ' and ' $Y$ ' axis and was determined with the use of the following equation:

$$
\varphi=\tan ^{-1}(V / U)
$$

where $V$ is the y-axis velocity, and $U$ is the x-axis velocity

The heading angle is shown in Figure 3.9:

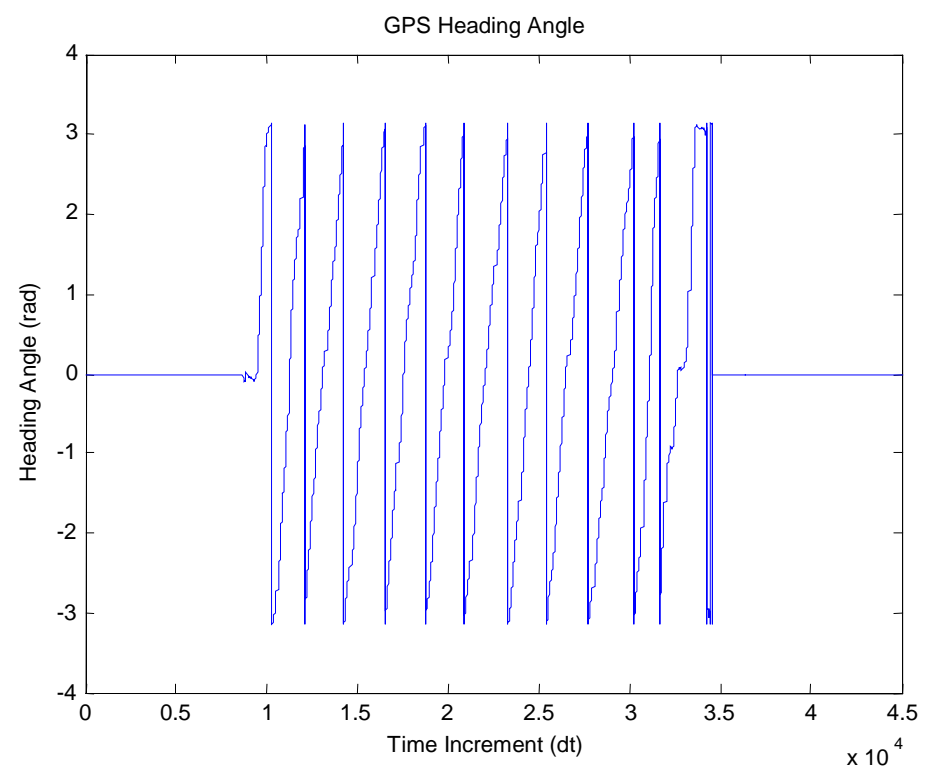

Figure 3.9: GPS Heading Angle

To calculate the roll and pitch angle of the aircraft using the GPS, a reference acceleration vector in the ECEF coordinate frame is first determined. This reference vector is constructed using the acceleration by differencing the present, $(k)$, and a priori, 
$(k-1)$, values of the GPS velocity. This reference vector represents the aircraft under the condition that there is no roll or pitch angles. The derivative of the velocity to obtain the acceleration is then determined by the following:

$$
a_{G P S}=\dot{v}=\frac{v_{G P S}(k)-v_{G P S}(k-1)}{d t}
$$

where $a_{G P S}$ is the GPS acceleration in ECEF, $v$ is the GPS velocity, and $d t$ is the time increment.

The GPS acceleration vector is then aligned with the $\mathrm{x}$ and $\mathrm{y}$ body-axis about the ECEF z-axis generating the reference acceleration $r_{x}$ and $r_{y}$. The reference angles are rotated about the z-axis due to the fact that the reference frame is under the condition that there is no roll or pitch angles causing the $\mathrm{x}, \mathrm{y}$, and z-axes to be orthogonal about the ECEF z-axis. The $r_{x}$ and $r_{y}$ transformations are defined as:

$$
\begin{aligned}
& r_{x}=-\left(\cos (\varphi) a_{G P S_{-} x}+\sin (\varphi) a_{G P S_{-} y}\right) \\
& r_{y}=-\left(-\sin (\varphi) a_{G P S_{-} x}+\cos (\varphi) a_{G P S_{-} y}\right)
\end{aligned}
$$

Since the aircraft is moving in the body frame relative to the ECEF frame, the IMU's accelerometers do not feel the effects of gravity. Therefore to compensate the GPS ECEF acceleration, the reference acceleration is calculated as follows:

$$
r_{z}=g-a_{G P S_{-} z}
$$

This transformation can be seen graphically in Figure 3.10, where $\mathrm{x}$ and $\mathrm{y}$ are the body axis and $y^{\prime}$ and $x^{\prime}$ is the ECEF axis: 


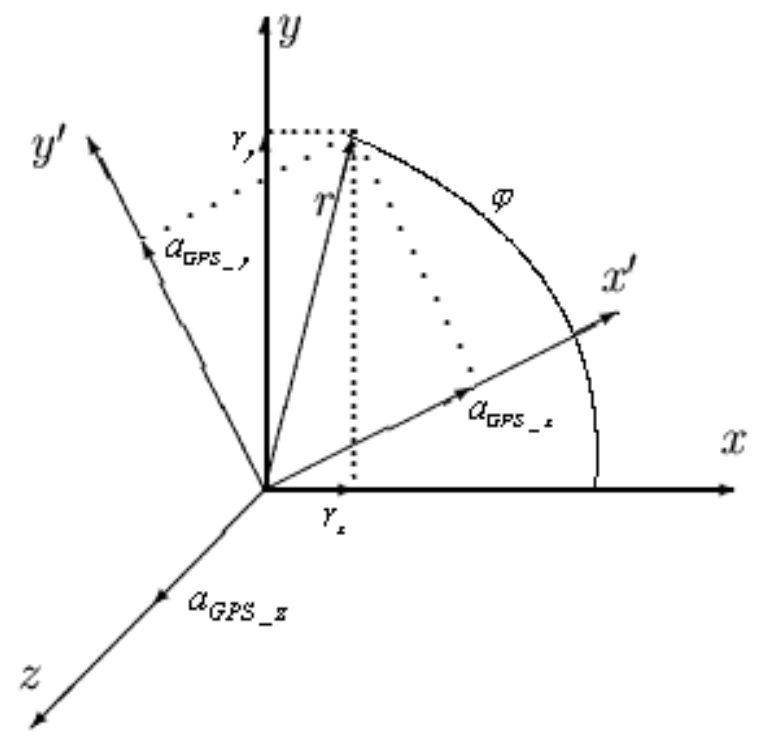

Figure 3.10: Rotation about the z-Axis to Align the ECEF Coordinate Frame with the Body Axis

With the reference vector constructed, the attitudes can then be defined and calculated with the following set of equations:

$$
\begin{gathered}
\sigma_{\theta}=\frac{r_{x} a_{x}+r_{z} \sqrt{r_{x}^{2}+r_{z}^{2}-a_{z}^{2}}}{r_{x}^{2}+r_{z}^{2}} \\
\theta=\tan ^{-1}\left(\frac{\sigma_{\theta} r_{z}-a_{x}}{\sigma_{\theta} r_{z}}\right) \\
r_{\theta}=r_{x} \sin (\theta)+r_{z} \cos (\theta) \\
\sigma_{\phi}=\frac{r_{y} a_{y}+r_{\theta} \sqrt{r_{y}^{2}+r_{\theta}^{2}-a_{y}^{2}}}{r_{y}^{2}+r_{\theta}^{2}} \\
\phi=\tan ^{-1}\left(\frac{\sigma_{\phi} r_{Y}-a_{y}}{\sigma_{\phi} r_{\theta}}\right)
\end{gathered}
$$

Figure 3.11 shows the raw GPS attitude estimate which has a great deal of noise on in the signal, although it still follows the same trends as the Vertical Gyro data. The noise from the GPS attitudes is not an issue, for the EKF will compensate for the negative effects of the noise while still correcting for the drift error associated with the INS. 

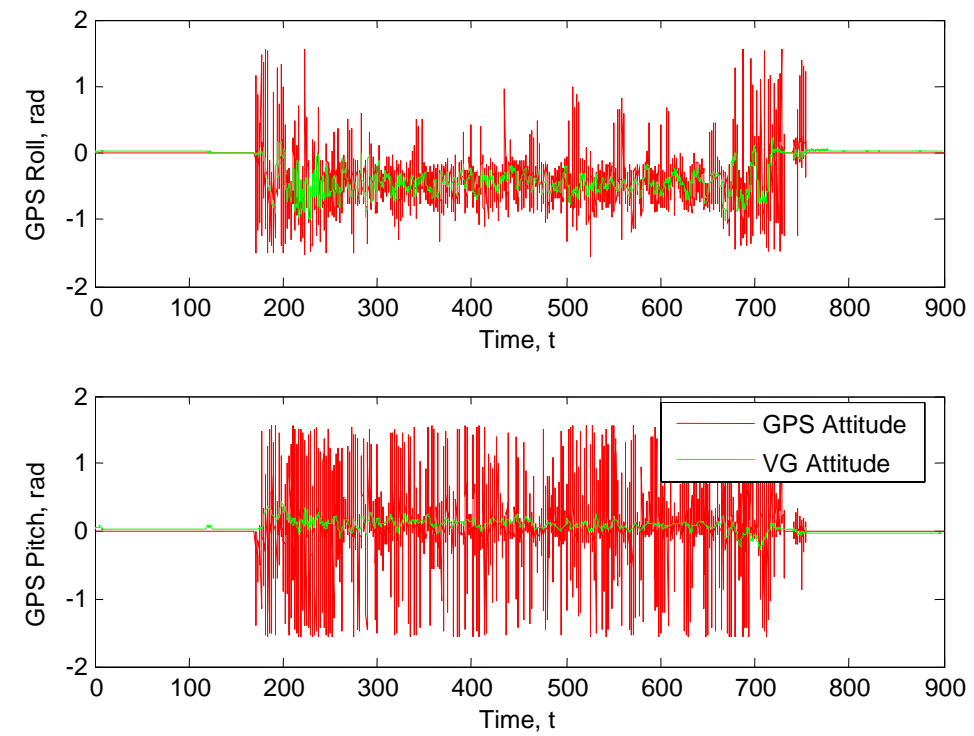

Figure 3.11: GPS vs Vertical Gyro Attitude

To further show that the GPS attitude does actually depict the aircraft roll and pitch attiude, the noisy values are sent through a low-pass butterworth filter, offline, as seen in Figure 3.12, then plotted against the vertical gyro data. This representation shows distinct evidence that this method of estimating the attitude is effective.
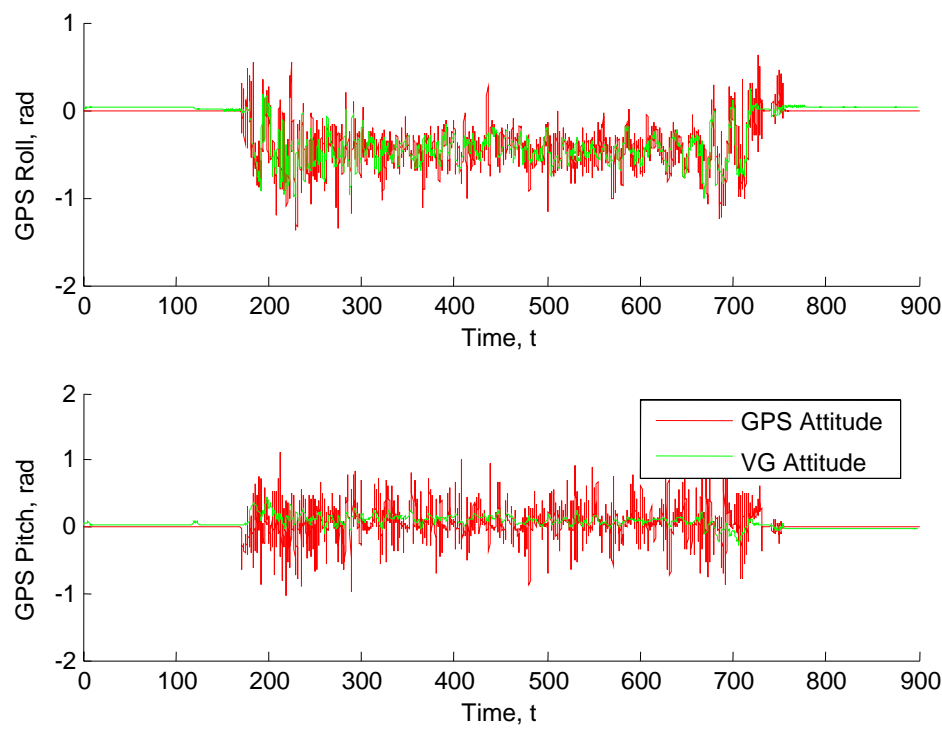

Figure 3.12: GPS vs Vertical Gyro Attitude 


\subsection{Kalman Filter}

The following sections describe the calculations and theory that make up the Kalman filter. Section 3.5.1 describes the simple Kalman filter, which is only used for linear systems, while section 3.5.2 discusses the Extended Kalman filter for non-linear applications.

\subsubsection{Introduction to Kalman Filtering}

The calculations that make up the filtering process can be separated into two steps depicted in Figure 3.13; time and measurement update. The time update, or "prediction" step projects the states and error covariance values ahead in time using the dynamic model of the system while the measurement update or "correction" step then calculates the Kalman gain. This value is then used to correct the error in the states and finally update the error covariance matrix to be input back into the "prediction" equations. The filtering calculations are discussed in greater detail in the following paragraphs. The following description is based around the fusion of GPS/IMU data, although a more general breakdown of the Kalman filter can be seen in greater detail in [5], [40], and [41].

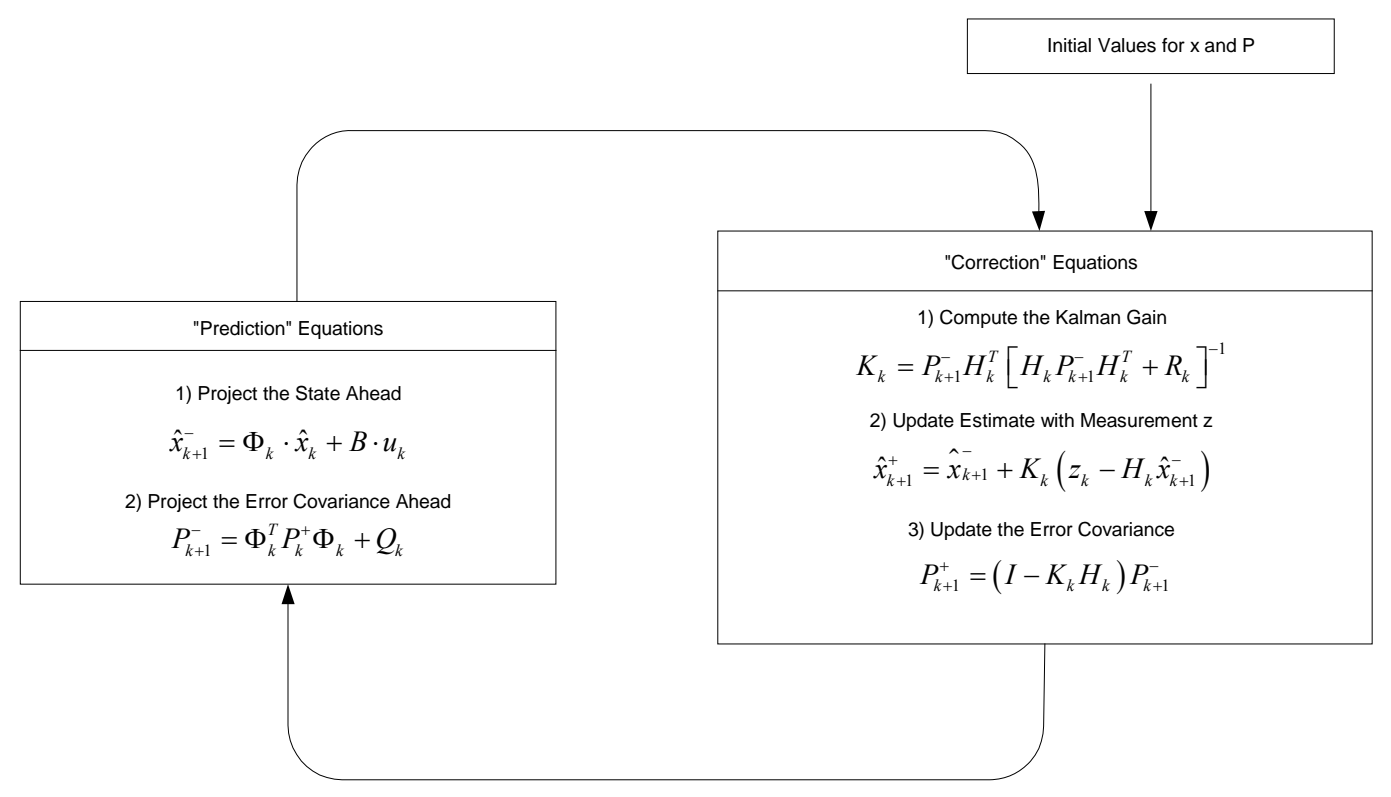

Figure 3.13: Kalman Filter Sequence 


\subsubsection{Time Update - "Prediction"}

The "prediction" step is composed of two calculations, the predicted state and covariance calculations, in which the predicted state update is composed of the system dynamics of the INS, which was discussed in detail in Section 3.3.1. The state update determines the a priori estimate of the state vector, ' $x$,' at time $\mathrm{k}$, knowing the previous values for ' $\mathrm{x}$ ' at time $\mathrm{k}-1$.

Step two of the time update section determines the value of the a priori estimate of the error covariance matrix with the use of the Riccati equation discussed further in the next section. By utilizing the state transition matrix of the dynamic system (STM, $\Phi$ ) and the covariance of the dynamic disturbance (Q), the error covariance matrix, 'P,' can be calculated as follows:

$$
P_{k+1}^{-}=\Phi_{k} P_{k}^{+} \Phi_{k}^{T}+Q_{k}
$$

The error covariance a priori estimate takes into account that there is a known error/noise in the input and model of the system which causes the signal to drift or decay over time from the actual value. Figures of the INS output in Section 3.3.2 are good examples of the system's decay over time.

The covariance matrix associated with the dynamic disturbance or model/input noise, $\left(Q_{k}\right)$, estimates the noise that is directly associated with the error in the dynamic model as well as the noise of the sensor measurement. Equation (3-38) calculates the estimated value of the model/input covariance matrix, which contributes to the uncertainty of the dynamic system at each time step.

$$
Q_{k-1}=\Phi_{k-1} Q_{w} \Phi_{k-1}^{T}
$$

where $Q_{w}$ is the power spectral density (PSD) of the sensor.

The PSD of the sensor input signals is called white noise due to the fact that it has the same error at all frequencies as a comparison to the frequency spectrum of white light. The PSD of the sensor error can be assumed to be zero mean and measured by conducting a variance analysis on the signal at a steady state response as follows:

$$
E[\omega(t)]=0, E\left[\omega(t) \omega^{T}(t)\right]=Q_{w}(t)
$$


where the first term on the left represents the zero mean random vector of the sensor input while the second expression represents the PSD analysis of the white noise associated with the sensor data to generate a matrix associated with each sensor error.

\subsubsection{Measurement Update - “Correction”}

The "correction" step is composed of three calculations; computation of the Kalman gain, update of the estimated states, and the error covariance a posteriori update. Computation of the Kalman gain is based on the algebraic (filter) Riccati equation $(\mathrm{ARE})^{10}$, which is a discrete recursive equation to calculate the error covariance, $P$. The Riccati equation, or the matrix quadratic equation, in its general form is as follows:

$$
P_{k}=\Phi_{k}^{T}\left[P_{k+1}-P_{k+1} H_{k}^{T}\left(H k P_{k+1} H_{k}^{T}+R_{k}\right)^{-1} H_{k}^{T} P_{k}\right] \Phi_{k}+Q_{k}
$$

where $P$ is the error covariance matrix, $H$ is the system output matrix, and $R$ is the covariance matrix of the measurement noise.

By manipulating the Equation (3-38, the a priori covariance matrix, a posteriori covariance matrix, and the Kalman gain equations are obtained. The a priori calculation in Section 3.5.1.1 is used to predict an estimate of the error covariance, $P$, determined from the system model at the new time step ' $\mathrm{k}$ ' using the a posteriori value from the previous time step, ' $\mathrm{k}-1$ '.

$$
P_{k+1}^{-}=\Phi_{k}^{T} \overbrace{\left[P_{k}^{+}\right]}^{\text {a posterori }} \Phi_{k}+Q_{k}
$$

With the a priori estimate of the obtained the Kalman gain can be calculated which also begins the measurement update portion of the filter. Again referring to the Riccati equation the Kalman filter gain calculation can be determined as follows:

$$
\begin{aligned}
& P_{k+1}^{+}=\Phi_{k}^{T}[P_{k+1}^{-}-\overbrace{P_{k+1}^{-} H_{k}^{T}\left(H_{k} P_{k+1}^{-} H_{k}^{T}+R_{k}\right)^{-1}}^{\text {Kalman Gain }} H_{k}^{T} P_{k+1}^{-}] \Phi_{k}+Q_{k} \\
& K_{k}=P_{k+1}^{-} H_{k}^{T}\left(H_{k} P_{k+1}^{-} H_{k}^{T}+R_{k}\right)^{-1}
\end{aligned}
$$

Similar to the system model/input covariance matrix, $Q_{k}$, the covariance matrix of the measurement noise is also zero mean white noise $\left(R_{k}\right)$, which is determined as follows: 


$$
E[v(t)]=0, E\left[v(t) v^{T}(t)\right]=R_{k}(t)
$$

where $v$ is the noise in reference to the measurement data used in the Kalman filter.

The state estimate is next compensated with the use of the Equation (3-45). This compensation is conducted by first computing the residual between the measurement (actual) value and the state estimated value. The amount of residual used to correct the estimated state is determined by multiplying the Kalman gain by the residual.

$$
\hat{x}_{k}^{+}=\hat{x}_{k}^{-}+K_{k}\left(z_{k}-H_{k} \hat{x}_{k}^{-}\right)
$$

where $z$ is the measurement value.

At this point the a posteriori covariance matrix is calculated by plugging the Kalman gain back into the Riccati equation to obtain the updated value for the covariance.

$$
\begin{gathered}
P_{k+1}^{+}=\Phi_{k}^{T} \overbrace{\left[P_{k+1}^{-}-P_{k+1}^{-} H_{k}\left(H_{k}^{T} P_{k+1}^{-} H_{k}+R_{k}\right)^{-1} H_{k}^{T} P_{k+1}^{-}\right]}^{\text {a posteriori Covariance Matrix }} \Phi_{k}+Q_{k} \\
P_{k+1}^{+}=\left(I-K_{k} H_{k}\right) P_{k+1}^{-}
\end{gathered}
$$

This set of calculations is then iterated over the time duration to improve the states of the system. The Kalman filter's ability to improve the states can once again be attributed to the Riccati equation, which is a recursive least squares solution to minimize the performance index, $J$, of the system. A good analogy for the performance index can be referring to it as the "cost" that it takes to put the estimated state back on the optimal path. As the performance index is minimized the overall accuracy of the system in improved.

Figure 3.14 depicts the path that the state estimate and covariance matrix take between two increments in discretized time. As seen from the following figure, the state transition matrix estimates a new value for the state, $\hat{x}_{k}^{-}$, which is then corrected by the Kalman gain to generate a more accurate value for the state, $\hat{x}_{k}^{+}$. 


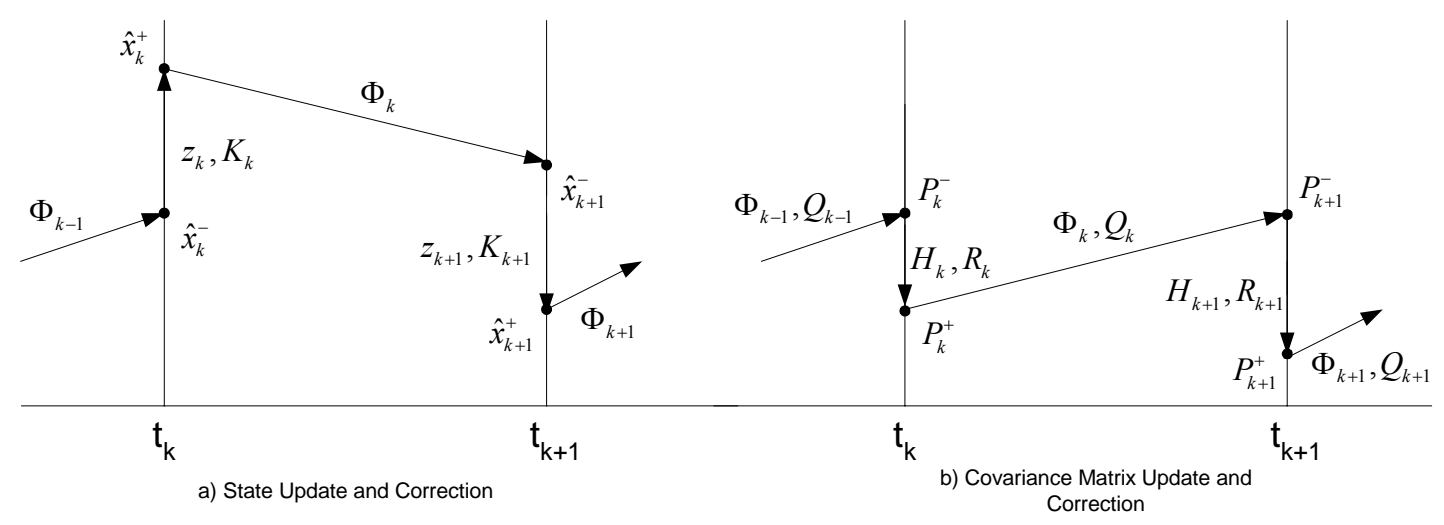

Figure 3.14: Kalman Filter Progression of States and Covariance Matrix

The effect of the error covariance has on the system determines the amount of measurement that gets used to update the state estimates. As the error covariance decreases, the position of the estimate is trusted more, which doesn't allow as much of the measurement to update the estimate. Inversely, if the error covariance increases, the estimated position is trusted less allowing the measurement position to correct the estimate. Ideally, as the system progresses over time the value for the error covariance should get close to zero. This reduction is mainly due to the 'actual' measurement values to maintain the accuracy of the system. Although the model/input noise covariance, Q, gets added into the error covariance matrix at each time increment limiting the reduction.

\subsubsection{Extended Kalman Filter}

The Kalman filter is based on the principle that the dynamic model of the system is linear. Most systems of any level of complexity are composed of non-linear elements, which draw the need for the extended Kalman filter (EKF). Schmidt introduced the first application of the EKF during the NASA Ames research discussed earlier. This method of the Kalman filter has also been referred to as the "Kalman-Schmidt" filter.

It must be noted that by utilizing an EKF increases the level of complexity of the system by first creating the need to linearize the differential equations and then the update of the system and input matrix at each time step making the system time variant. These additional calculations also drastically increase the amount of computational load on the system. 
Other than the previous stated criteria, the EKF is relatively similar to the Kalman filter, as seen in Figure 3.15, the Kalman gain, a priori and a posteriori covariance matrix calculations are the same as the previous method. An application of the EKF within the context of this thesis topic is the linearization of the INS attitude determination. The attitude calculations discussed in Section 3.3.2 involve the use of a time variant linearized dynamic model that is derived from the non-linear dynamic model seen below.

$$
\begin{gathered}
\hat{x}_{k+1}^{+}=f\left(\hat{x}_{k}^{-}\right)+w_{k} \\
z_{k}=h\left(\hat{x}_{k}^{-}\right)+w_{k}
\end{gathered}
$$

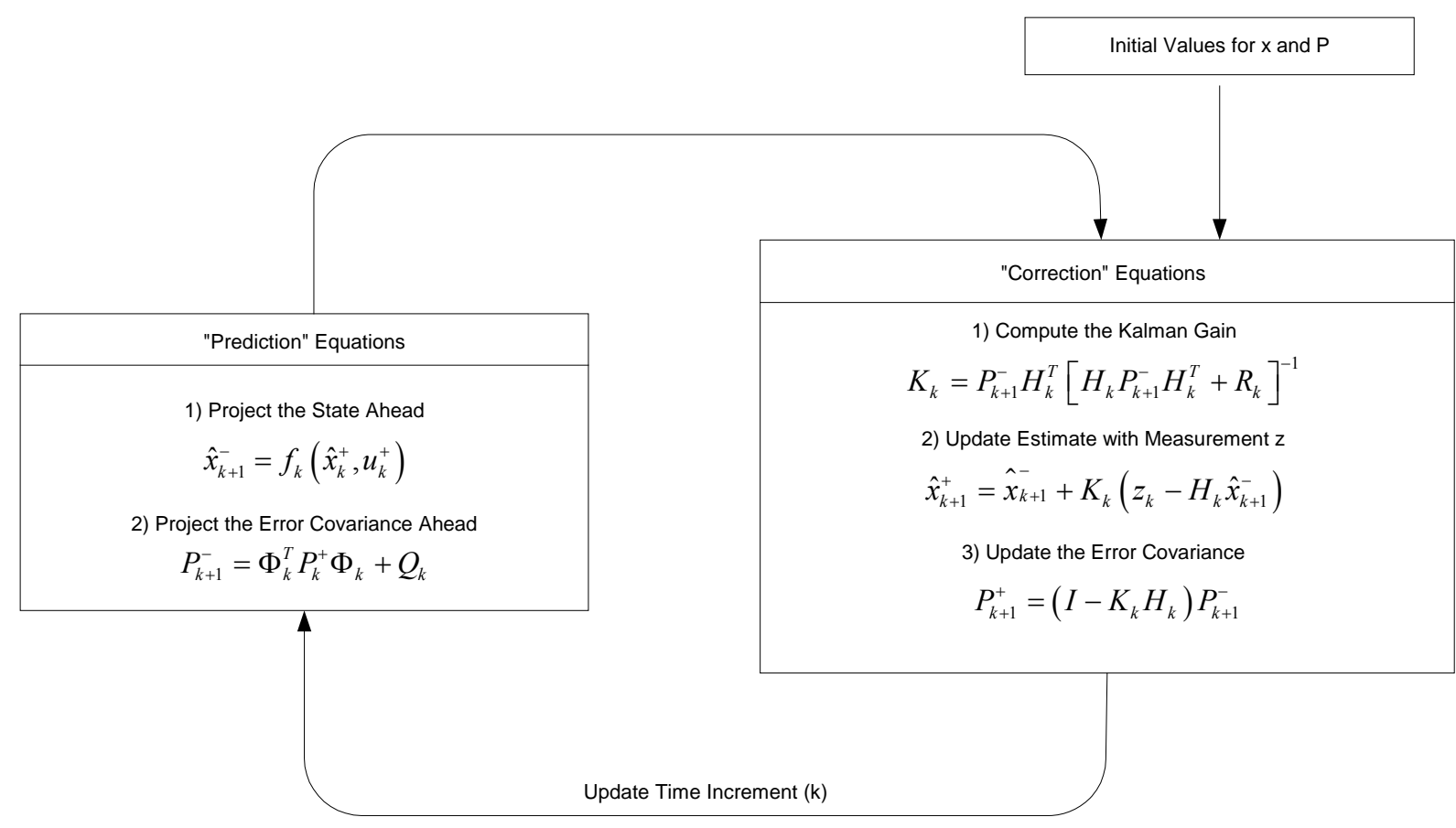

Figure 3.15: Extended Kalman Filter (EKF) Sequence

Referring back to the discussion in section 2.2.2, the extended Kalman filter is not an optimal filter due to the linearization of the system model. This is described in greater detail by referring back to the Ricotti equation, in which it is responsible for estimating and minimizing the error of linear systems. By linearizing the system model by means of the Taylor series, additional error is induced causing the filter to improperly estimate the error covariance matrix. The additional error does not pose a great issue, as the EKF is still a highly effective tool for estimating and reducing the system error. However this 
does also generate the need for a higher level of accuracy when determining the covariance matrices, which could cause the filter to diverge if not properly modeled 


\section{Chapter 4. Experimental Procedures}

\subsection{Overview of Experimental Procedures}

The experimental procedures within the context of this research project required the development of software algorithms that work with an array of sensor data to meet the goals and objectives set forth in Section 1.1. This chapter describes the hardware utilized in the collection of sensor data, the synchronization of the data, and the implementation of the Kalman filtering methods to achieve the previously stated objectives. Section 4.2 describes the first phase of this research project in which previously obtained flight data from the WVU formation flight research was utilized.

\subsection{WVU YF-22 IMU/GPS/Vertical Gyro Sensor Fusion}

The experimental procedures for this portion of the research project are composed of a small amount of sensor data manipulation, with the rest being the development of software algorithms integrating the various sensors to improve the position, velocity and attitude of the aircraft. Sensor data for this section of the research project was utilized from the WVU research YF-22 UAV's during the formation flight research discussed previously.

The first section, Section 4.2.1, contains the hardware description of the sensors used for the WVU YF-22 formation flight research project. This flight data is then utilized in the implementation of the various Kalman filtering software algorithms for the improvement of the aircraft parameters. The second section, Section 4.2.2 discuses the limitations of the system and corrective measures taken to achieve results with a higher level of precision and Section 4.2.3 focuses on the implementation of various Kalman filtering techniques on the formation flight GPS and IMU data.

\subsubsection{Hardware Used for the WVU YF-22 Attitude Improvement}

The following section describes the hardware used during the formation flight research conducted at WVU on three YF-22 test-beds. All data used within the context of this section was previously recorded flight data, meaning no additional data acquisition 
was required for this portion of the research topic. This hardware description provides an insight and background of the YF-22 test vehicles described in Table 4-1.

Table 4-1: WVU YF-22 Test Vehicles/Sensor-Componant Package

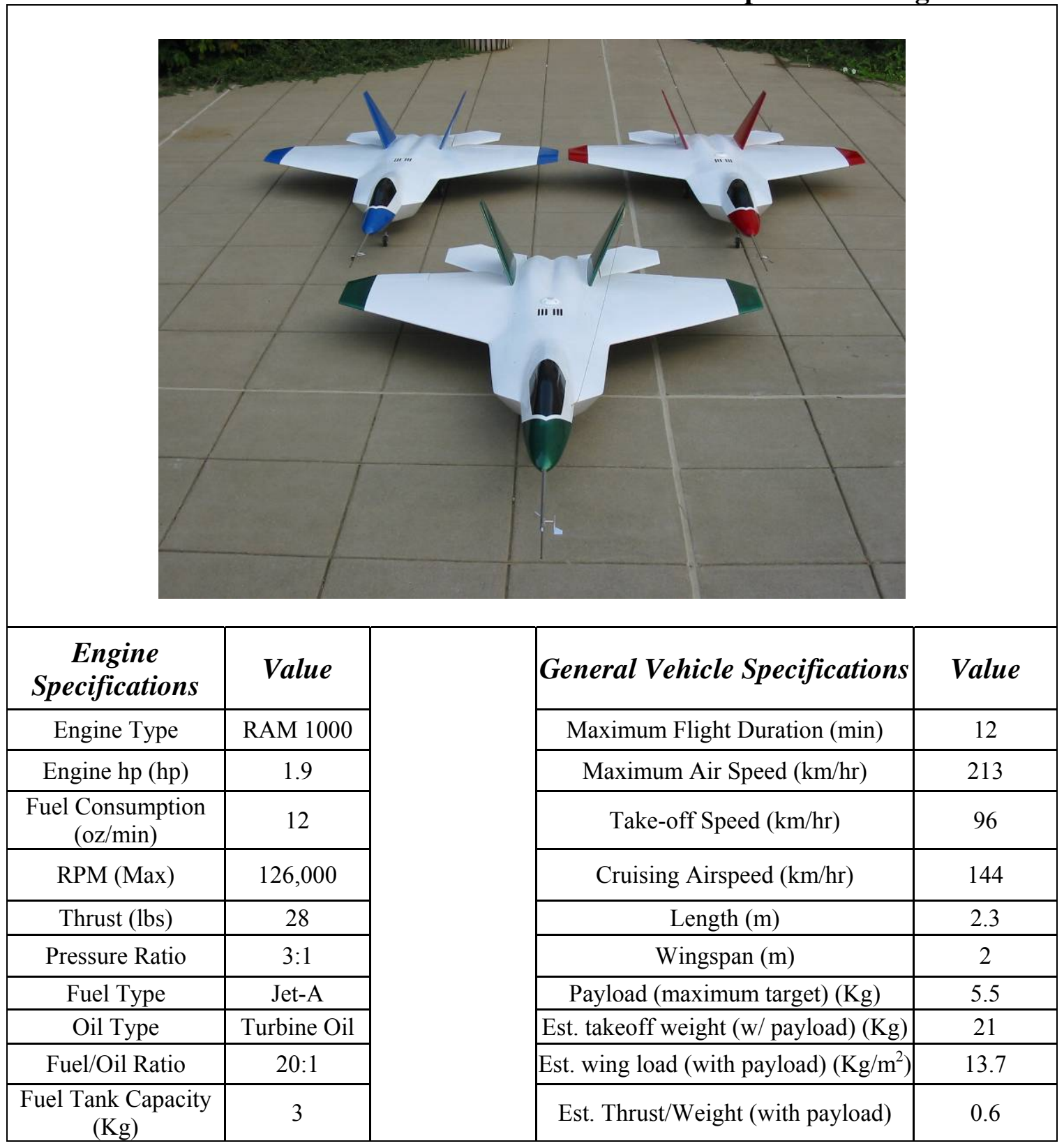

The WVU YF-22 test vehicles were designed, constructed, maintained, and operated by faculty and students at WVU. The main fuselage is constructed from fiberglass, while the wings and control surfaces have a foam core, sheeted with balsa wood, then covered in fiberglass and painted to the three schemes shown in Table 4-1. The vehicles are powered by RAM 1000 jet engines producing approximately $28 \mathrm{lbs}$. of 
thrust allowing the vehicles to achieve a maximum air speed of $213 \mathrm{~km} / \mathrm{hr}(132 \mathrm{mph})$ and a cruising velocity of $144 \mathrm{~km} / \mathrm{hr}(89 \mathrm{mph})$.

The sensor and component package implemented on the formation flight aircraft in relation to this research consists of an IMU, GPS, Vertical Gyro, and flight computer. Table 4-2 illustrates the component mounting locations of the IMU, GPS, Vertical Gyro, flight computer, and power supply, in which each component is discussed in detail throughout the following sections. Additional sensors and components were implemented on the aircraft (as presented in Table 4-2), but have no immediate relevance to this effort.

Table 4-2: Sensor Specifications and Component Mounting

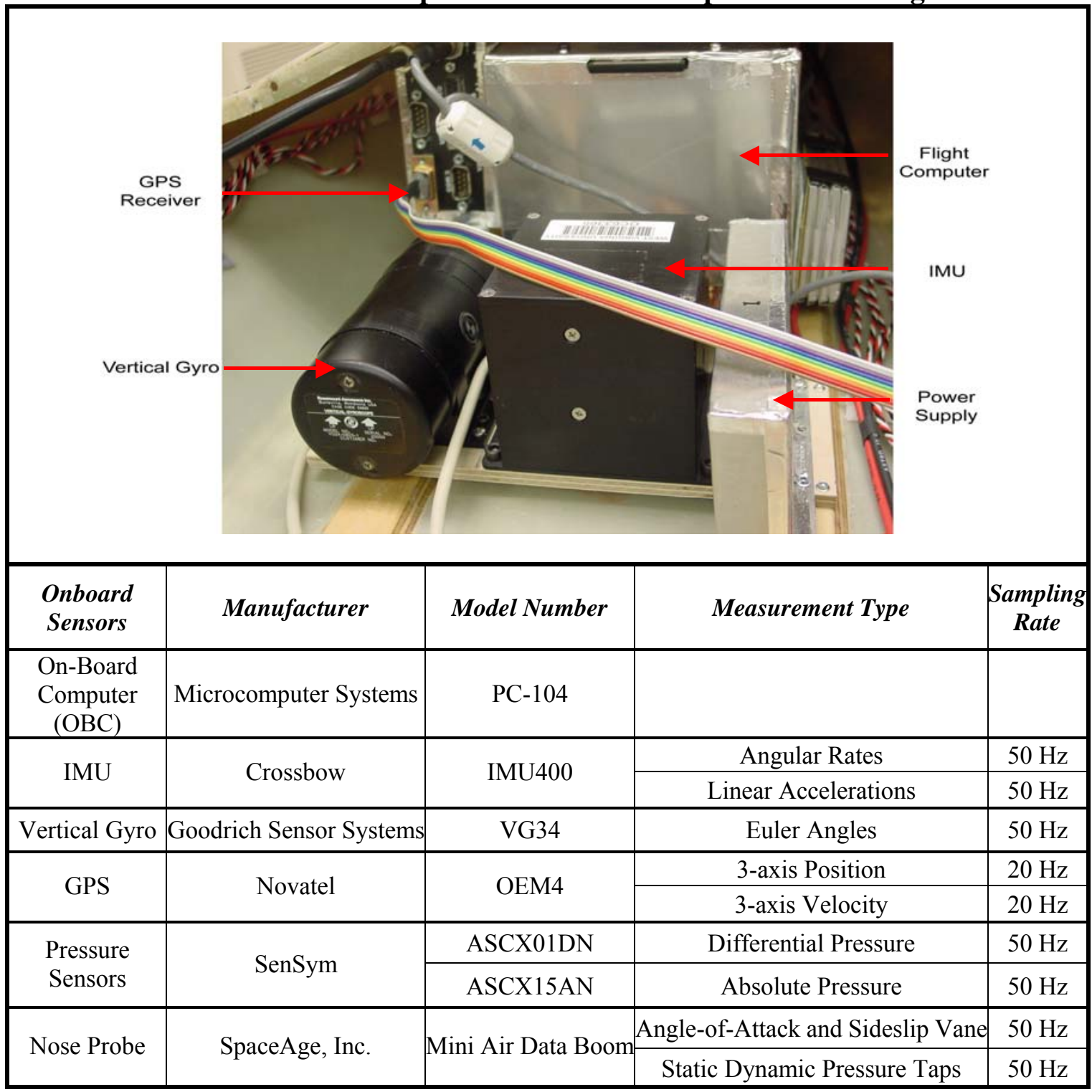




\subsubsection{Formation Flight On-Board Computer (OBC)}

The OBC, shown in Figure 4.1, was based on a $300 \mathrm{MHz}$ PC-104 stack computer system, which contained a CPU module, a data acquisition module, a power supply module, and supporting components. The PC-104 format was selected because of its reduced size, lightweight, and low power consumption ${ }^{2}$.

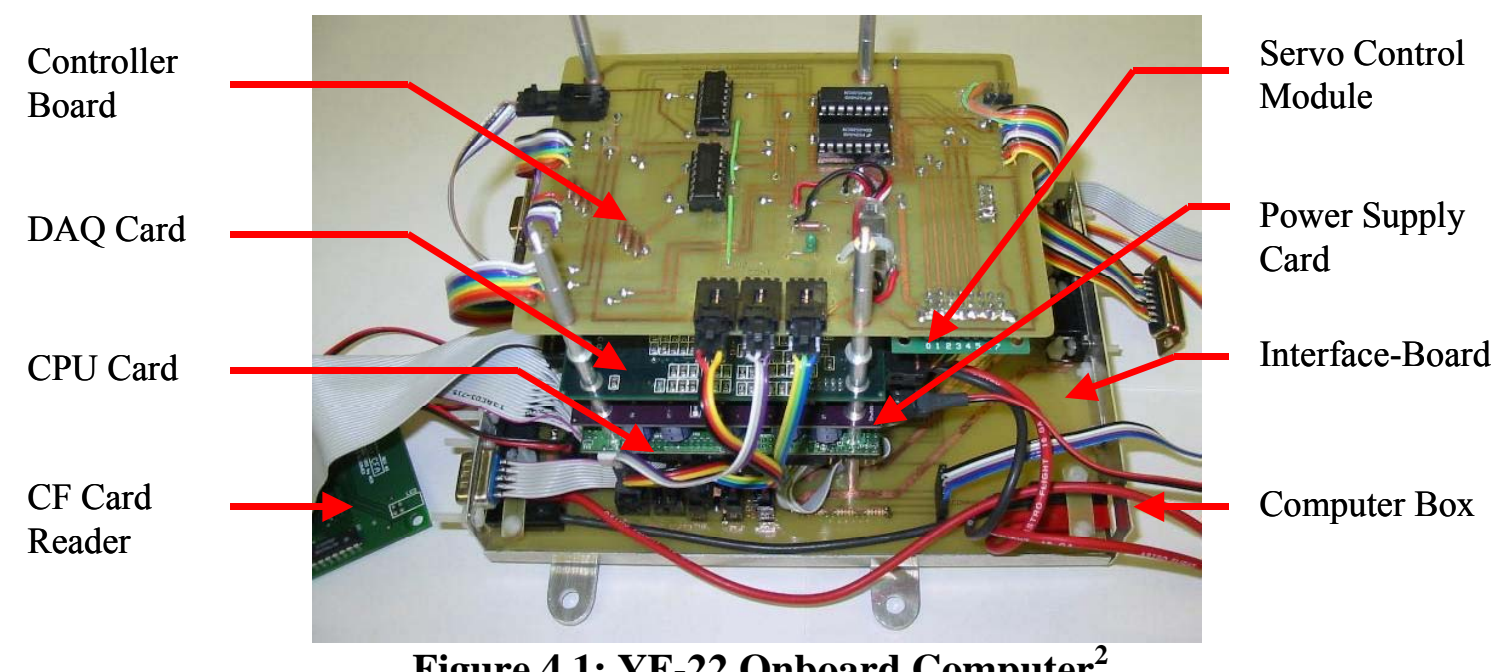

Figure 4.1: YF-22 Onboard Computer ${ }^{2}$

\subsubsection{NovAtel GPS Receiver}

A NovAtel OEM4 GPS receiver was used to measure the 3-axis position and velocity to meter level accuracy, which was read into the flight computer at a rate of 20 Hz. This data was required for each of the three YF-22 test-beds in order to achieve formation flight, in which position and distance information between the aircraft had to be obtained. While the flight computer recorded only the position and velocity information, the OEM4 receiver is capable of obtaining a variety of additional information depending on the firmware configuration purchased through NovAtel, including the ability to obtain raw pseudorange and ephemeral data, along with real-time kinematic (RTK) processing which allows the receiver to obtain position data at a level of either 2 or $20 \mathrm{~cm}$ accuracy. 


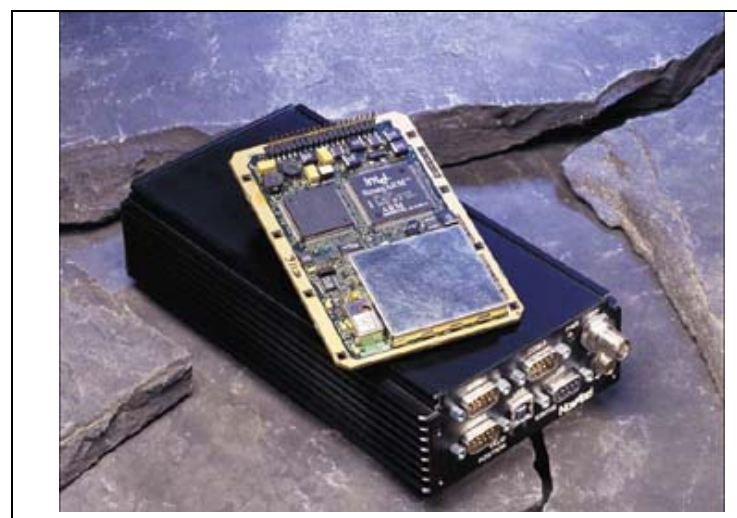

(a) NavAtel GPS Receiver

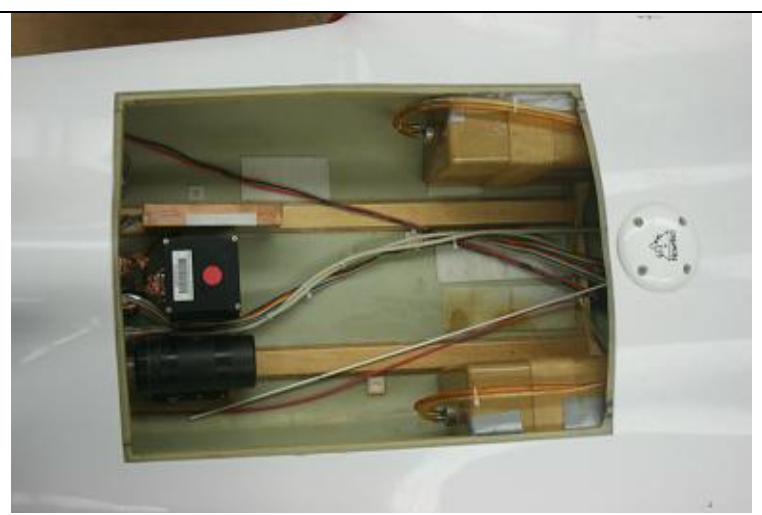

(b) GPS Antenna Location

Figure 4.2: NovAtel GPS

The selected GPS antenna was the GPS-511, also manufactured by Novatel, Inc., which offered desirable performance for airborne and high dynamic applications. The GPS antenna was mounted on the top section of the aircraft fuselage at the center of gravity (CG) location, as shown in Figure 4.2 (b), in relation to the IMU, Vertical Gyro, and power supply.

\subsubsection{Crossbow IMU}

The Crossbow IMU400CC-200 was selected for the formation flight research due to its lightweight, cost, and performance abilities. This component was a solid-state 6degree-of-freedom (6 DOF) inertial package intended for navigation and control, dynamic testing, and instrumentation applications. This system provided measurements of the angular rates and linear accelerations along three orthogonal axes. Fully compensated angular rate and acceleration outputs were provided in both analog and digital (RS-232) formats, which were recorded at a rate of $50 \mathrm{~Hz}$ by the flight computer. 


\begin{tabular}{|c|c|c|}
\hline Performance & Maximum Update Rate $(\mathrm{Hz})$ & 100 \\
\hline \multirow{2}{*}{ Angular Rate } & Range: Roll, Pitch, Yaw ( $\% / \mathrm{sec})$ & \pm 200 \\
\hline & Bias: Roll, Pitch, Yaw (\%/sec) & $< \pm 1$ \\
\hline \multirow{2}{*}{ Acceleration } & Range: $\mathrm{X}, \mathrm{Y}, \mathrm{Z}$ (g) & \pm 10 \\
\hline & Bias: $\mathrm{X}, \mathrm{Y}, \mathrm{Z}$ (mg) & $< \pm 12$ \\
\hline
\end{tabular}

\subsubsection{Goodrich Sensor Systems Vertical Gyro}

The vertical gyro attitudes were obtained by a Goodrich Sensor Systems brand, model VG34 vertical gyro, shown in Figure 4.3. This unit is Goodrich Sensor System's smallest vertical gyro, that produces highly accurate measurements in which the range is $\pm 90^{\circ}$ for the roll angle, with an accuracy of $\pm 1^{\circ}$, and $\pm 60^{\circ}$ for the pitch angle, also with an accuracy of $\pm 1^{\circ}$.

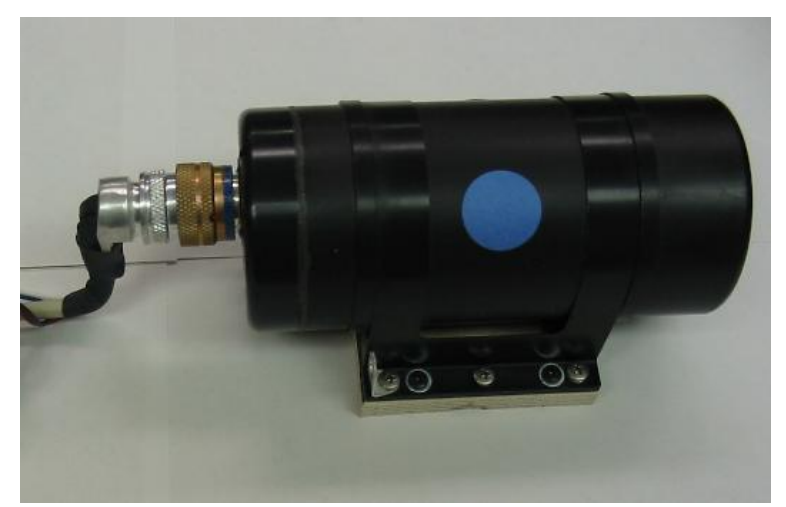

Figure 4.3: Goodrich Systems Vertical Gyro

The size, cost, and accuracy of the VG34 make it a an optimal choice for many additional high performance vehicles such as tracked wheeled and tracked armored vehicles, aircraft, helicopters, missiles, drones and remotely piloted aerial vehicles 


\subsubsection{Limitations/Corrections to the WVU Formation Flight Hardware Setup}

Although the data collected during the formation flight research project was actual flight data, which in many ways increases the validity of the data and results, also creates some limitations on the project at hand. Along with some of the limitations and restrictions of the collected data, there are also some additional corrective measures that have been conducted.

\section{GPS Position Data}

The GPS data collected during flight tests consisted of only position and velocity data, no pseudorange, or raw ephemeral data was collected. This restricts the Kalman filter to a loosely coupled system, which will be discussed in greater detail later in this chapter.

\section{Acquisition Rate}

The first issue between the IMU and GPS data being utilized is the difference in the sampling rates. The IMU samples were taken at $50 \mathrm{~Hz}$ while the GPS samples could be obtained at $20 \mathrm{~Hz}$, which causes the data to only match on increments of $0.1 \mathrm{~s}$. The optimal method for utilizing most of the flight data was to resample the IMU sensor information at $40 \mathrm{~Hz}$ and maintain the GPS data at $20 \mathrm{~Hz}$. This allowed the Kalman Filter to correct for the IMU's data every other time step instead of every fifth time step, as seen in Figure 4.4.

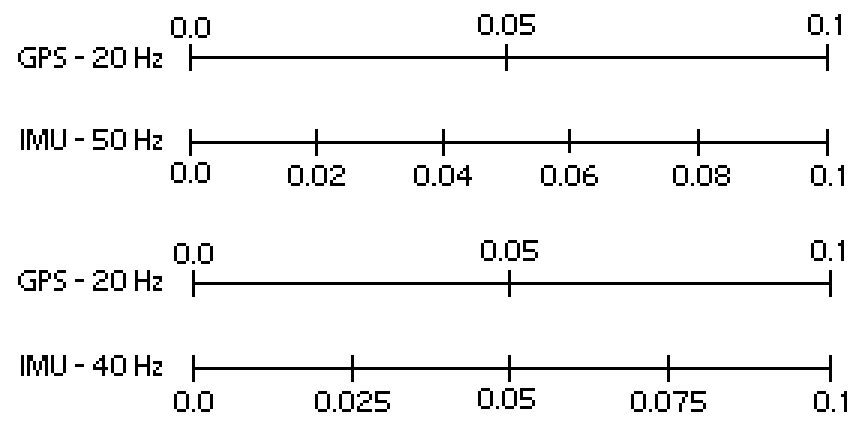

Figure 4.4: IMU vs. GPS Measurement Acquisition Rate

\section{Lever Arm Correction}

The lever arm correction compensates for the offset distance between the GPS antenna and the IMU sensor, which realigns the position and velocity data between the 
two sensors - back to the CG of the aircraft. This offset is caused by three main factors; a) the sensors cannot overlap each other, b) the GPS antenna must be placed on the fuselage of the aircraft, and c) to properly balance the aircraft the, sensors must be strategically placed within the aircraft. This correction was conducted by adjusting the relative acceleration for one point (the IMU), rotating about a second point (the GPS antenna, at the CG location). The lever arm correction was conducted with the use of the following ${ }^{40}$ :

$$
\begin{gathered}
a_{A}=a_{B}-\alpha \cdot r+\omega^{2} \cdot d \\
{\left[\begin{array}{l}
a_{x} \\
a_{y} \\
a_{z}
\end{array}\right]_{A}=\left[\begin{array}{c}
a_{x} \\
a_{y} \\
a_{z}
\end{array}\right]_{B}-\left[\begin{array}{ccc}
0 & -\alpha_{r} & \alpha_{q} \\
\alpha_{r} & 0 & -\alpha_{p} \\
-\alpha_{q} & \alpha_{p} & 0
\end{array}\right]\left[\begin{array}{c}
r_{x} \\
r_{y} \\
r_{z}
\end{array}\right]+\left[\begin{array}{ccc}
0 & -\omega_{r}^{2} & \omega_{q}^{2} \\
\omega_{r}^{2} & 0 & -\omega_{p}^{2} \\
-\omega_{q}^{2} & \omega_{p}^{2} & 0
\end{array}\right]\left[\begin{array}{l}
d_{x} \\
d_{y} \\
d_{z}
\end{array}\right]}
\end{gathered}
$$

where $\alpha$ is the angular acceleration, $\omega$ is the angular rate, and $r$ is the position offset from the GPS antenna location to the IMU location.

The angular acceleration was then determined by differencing the angular rate from the time step $k$, with the previous time step $k-1$. The values for the angular velocities and accelerations are then placed into a skew matrix so that each directional component of the acceleration will be corrected.

\section{Bad Measurement Determination}

Bad measurements from degraded GPS signal data were also accounted for when analyzing flight data. The main periods for when the GPS data precision deteriorated during the WVU formation flight test experiments was during high bank angle maneuvers, as depicted in Figure 4.5, although while testing the validation data there was one instance when a total signal loss was discovered as seen in Figure 4.6. 


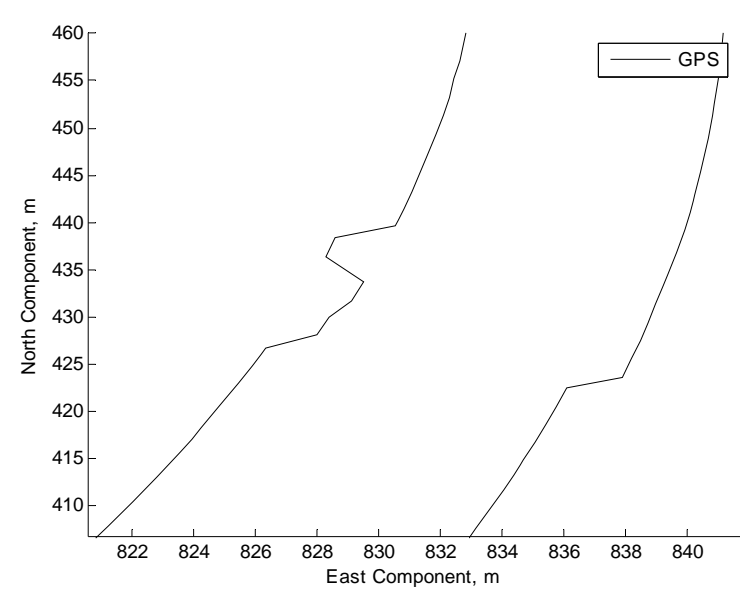

Figure 4.5: GPS Position (Magnified)

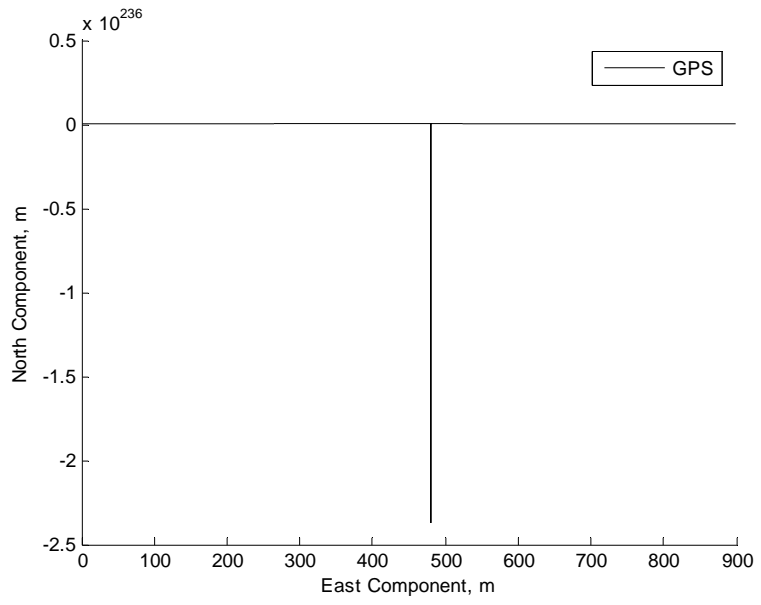

Figure 4.6: GPS Position - Instantaneous Signal Loss

While the filtering process compensated for the GPS error, the elimination of measurements that were unrealistic produced higher quality and more accurate results. Since the vehicles main dynamic movement is along the longitudinal axis, a significant lateral maneuver was unrealistic and not probable. Therefore whenever this type of movement was detected, the measurement was neglected and only the INS measurement utilized. Although the INS system is susceptible to drift effects, the short-term use of only the INS values is more precise than the filtered estimates.

This correction was accomplished by comparing the Kalman filter residual calculation, $\left(z_{i}-H_{i} \hat{x}_{i-1}\right)$, at time ' $k$ ' with the standard deviation. When the value of the residual exceeds a set threshold value of the standard deviation, the GPS value for that given time increment is omitted and the IMU's dynamic model computes the transition to the next increment.

\subsubsection{Software Used for the Integration of GPS/INS}

Within the context of creating Kalman filtering algorithms for the fusion of the YF-22 formation flight data, four methods were implemented, in which each method has benefits and drawbacks to be further discussed throughout this section. 


\subsubsection{Fusion of IMU/GPS/Vertical Gyro data}

This method fuses the GPS position and velocity, INS position and velocity, and the Vertical Gyro attitudes. The fusion of this method uses the GPS and INS components of position and velocity as the states within the Kalman filter, in which the benefit of this is to remove the drift within the INS and the signal errors in the GPS components of the position and velocity. The attitudes from the Vertical Gyro are used so that there is no need for non-linear modeling, allowing the use of the Kalman filter.

The algorithm for implementing the first method is composed of three main functions:

1) Data Preprocessing

2) INS Calculations

3) Kalman Filter Implementation

The data preprocessing is composed of the acquisition rate correction (discussed in the previous section) and the initialization of the filtering parameters.

The initialization of the filter is composed of the initial values of the state vector and error covariance matrix as well as the setting up of the system model/input and measurement noise covariance matrices, $Q$ and $R$ respectively. Initially the $6 x 6$ error covariance matrix, $P$, was set to zeros since the exact initial position was known and the initial state vector, $\hat{x}_{0}$, was also set to zeros in which both parameters got updated after each filtering iteration.

The measurement noise covariance matrix was determined by obtaining the variance of the GPS position and velocity measurements over a steady state period of time. Figure 4.7 depicts an example of the period in which the variance was obtained. 


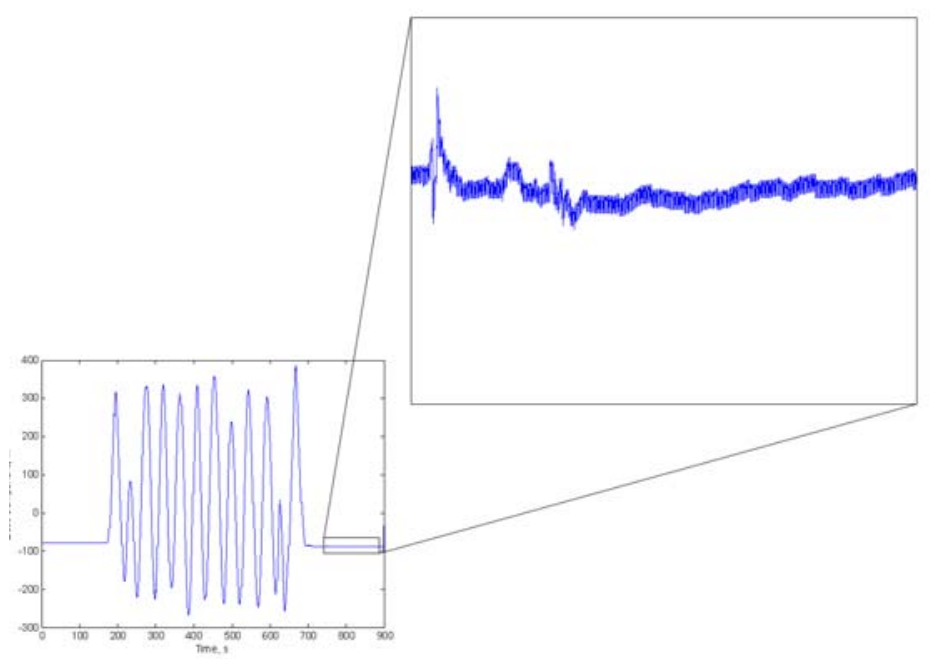

Figure 4.7: Steady State Time Period for Variance Calculation

These values were then inserted into a diagonal matrix respectively to generate the measurement noise covariance matrix of the GPS data:

$$
\begin{gathered}
R=\left[\begin{array}{cccccc}
\sigma_{x_{\text {Norh h }}}^{2} & 0 & 0 & 0 & 0 & 0 \\
0 & \sigma_{y_{\text {East }}}^{2} & 0 & 0 & 0 & 0 \\
0 & 0 & \sigma_{z_{\text {Down }}}^{2} & 0 & 0 & 0 \\
0 & 0 & 0 & \sigma_{u_{\text {North }}}^{2} & 0 & 0 \\
0 & 0 & 0 & 0 & \sigma_{v_{\text {East }}}^{2} & 0 \\
0 & 0 & 0 & 0 & 0 & \sigma_{w_{\text {Down }}}^{2}
\end{array}\right]= \\
{\left[\begin{array}{cccccc}
0.0022 & 0 & 0 & 0 & 0 & 0 \\
0 & 0.0017 & 0 & 0 & 0 & 0 \\
0 & 0 & 0.0046 & 0 & 0 & 0 \\
0 & 0 & 0 & 2.0988 \mathrm{e}^{-4} & 0 & 0 \\
0 & 0 & 0 & 0 & 1.7912 \mathrm{e}^{-4} & 0 \\
0 & 0 & 0 & 0 & 0 & 5.3837 \mathrm{e}^{-4}
\end{array}\right]}
\end{gathered}
$$

As discussed previously in this chapter, the filtering approach was restricted to a 'loosely' coupled system due to the lack of raw GPS data. A 'loosely' coupled system, although easier to implement than a tightly coupled system, does not estimate the IMU calibration parameters (e.g. biases), leaving the estimation of these parameters to the system designer.

These IMU calibration parameters were set into the model/input covariance matrix, $Q$, and are used as a tuning parameter. If the system designer did not correctly 
estimate these parameters, a reduction in the performance of the filter could occur ${ }^{6}$. This is not the case for 'tightly' coupled systems, in which these values are properly modeled as discussed in Section 3.5.1.1.

As a starting point for the initialization of the model/input covariance matrix, any value could be selected so that the value is greater than zero, but less than one; to obtain starting values, the initial values for this method were determined the same way as the measurement covariance matrix by placing the IMU acceleration variance during a steady-state condition in a $6 x 6$ diagonal matrix for the position and velocity as seen below:

$$
Q=\left[\begin{array}{cccc}
0_{3 x 3} & 0_{1 x 3} & 0_{1 x 3} & 0_{1 x 3} \\
0_{3 x 1} & \sigma_{a_{x}}^{2} & 0 & 0 \\
0_{3 x 1} & 0 & \sigma_{a_{y}}^{2} & 0 \\
0_{3 x 1} & 0 & 0 & \sigma_{a_{z}}^{2}
\end{array}\right]=\left[\begin{array}{cccc}
0_{3 x 3} & 0_{1 x 3} & 0_{1 \times 3} & 0_{1 \times 3} \\
0_{3 x 1} & .004 & 0 & 0 \\
0_{3 x 1} & 0 & .0037 & 0 \\
0_{3 x 1} & 0 & 0 & .0046
\end{array}\right]
$$

The location for the position covariance values are intentionally left zero since they are a direct integration from the velocity, which values in the covariance matrix are composed of the variance of the acceleration data. The error associated with the velocity from the integration of the accelerometer and gyroscope data is directly passed on to the position. Meaning that by reducing the error in the velocity integration, the error in the position also decreases.

From these values if the model/system covariance were increased so that the value was closer to one, the system would closer mimic the measured value. Inversely, as the covariance approaches zero, the system would closer mimic the estimated values. After the initial system has been established these values are adjusted to suit the needs of the system. This tuning is explained and discussed further in the next chapter.

The INS calculations, with the exception of the integration of the attitudes, are discussed in detail in section 3.3.1. Again, the integration of the attitudes was neglected for this method since the vertical gyros attitude values were used. This allows the INS calculations to begin at equation (3-24) with the DCM being calculated using the vertical gyro's attitudes. 
With the data preprocessing and initialization complete, the Kalman filter can be implemented to improve the position and velocity data. Figure 4.8 illustrates a block diagram of the data flow throughout the filter resulting in the corrected data.

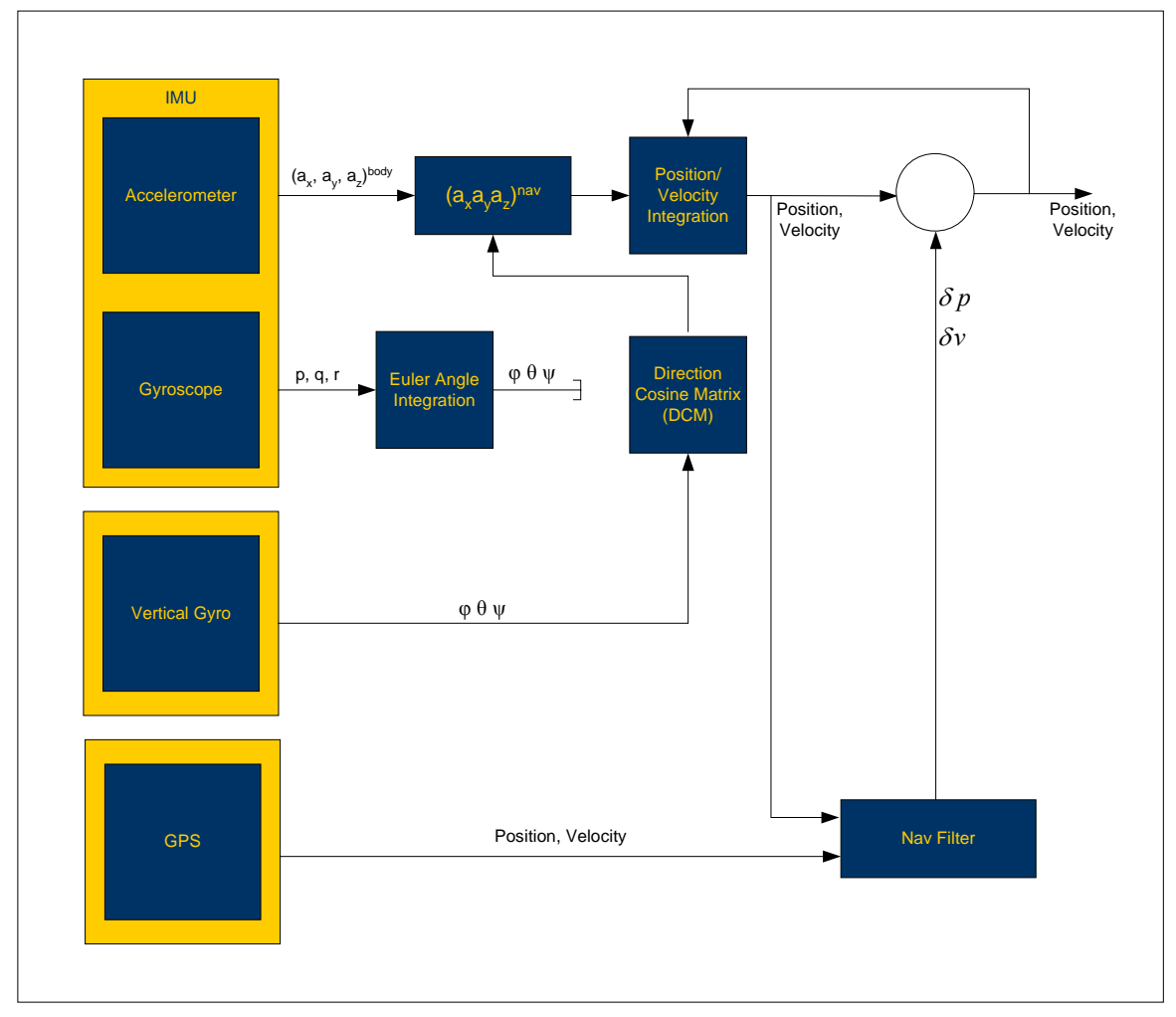

Figure 4.8: Block Diagram of a GPS Aided INS/Vertical Gyro

With the INS calculations, GPS, and vertical gyro data already discussed, the focus shifts to combining the data from the three sources in the proper manner so that the filter is implemented correctly. With the initial values set, the "prediction" step of the Kalman filtering process can be conducted in which the a priori covariance matrix and new state estimates are obtained. Revisiting Figure 3.13 and tailoring these equations to this specific application; Table 4-4 is constructed which distinctly defines each expression in the filtering process.

Table 4-4: "Prediction" Equations (Method I)

\begin{tabular}{|c|c|c|c|c|}
\hline & Nomenclature & Symbol & $\begin{array}{c}\text { Matrix } \\
\text { Size }\end{array}$ & Values \\
\hline $\begin{array}{c}\text { 1) Project the State Ahead } \\
\hat{x}_{k+1}^{-}=\Phi_{k} \cdot \hat{x}_{k}+B \cdot u_{k}\end{array}$ & System States & $\hat{x}_{k+1}^{-}, \hat{x}_{k}$ & $6 x 1$ & {$\left[\begin{array}{c}x_{k, 3 x 1} \\
v_{k, 3 x 1}\end{array}\right]$} \\
\hline
\end{tabular}




\begin{tabular}{|c|c|c|c|c|}
\hline & STM & $\Phi_{k}$ & $6 \times 6$ & {$\left[\begin{array}{cc}I_{3 \times 3} & I_{3 \times 3} \cdot d t \\
0_{3 \times 3} & I_{3 \times 3}\end{array}\right]$} \\
\hline & Input Matrix & $B$ & $6 \times 3$ & {$\left[\begin{array}{c}0_{3 \times 3} \\
I_{3 \times 3} \cdot d t\end{array}\right]$} \\
\hline & Inputs & $u_{k}$ & $3 x 1$ & {$\left[a_{3 x 1}^{n a v}\right]$} \\
\hline \multirow{3}{*}{$\begin{array}{l}\text { 2) Project the Error Covariance } \\
\text { Ahead } \\
\qquad P_{k+1}^{-}=\Phi_{k}^{T} P_{k}^{+} \Phi_{k}+Q_{k}\end{array}$} & STM & $\Phi_{k}$ & $6 x 6$ & {$\left[\begin{array}{cc}I_{3 \times 3} & I_{3 \times 3} \cdot d t \\
0_{3 x 3} & I_{3 x 3}\end{array}\right]$} \\
\hline & $\begin{array}{c}\text { Model/Input } \\
\text { Covariance } \\
\text { Matrix }\end{array}$ & $Q_{k}$ & $6 \times 6$ & {$\left[\begin{array}{cc}\operatorname{diag}\left(\sigma_{r, 3 \times 3}^{2}\right) & 0_{3 \times 3} \\
0_{3 \times 3} & \operatorname{diag}\left(\sigma_{v, 3 \times 3}^{2}\right)\end{array}\right]$} \\
\hline & $\begin{array}{c}\text { Error } \\
\text { Covariance } \\
\text { Matrix }\end{array}$ & $P_{k+1}^{-}, P_{k}^{+}$ & $6 \times 6$ & {$\left[\operatorname{diag}\left(X_{6 x 6}\right)\right]$} \\
\hline
\end{tabular}

At this point the "correction" step was calculated in which the Kalman gain, Eq. (3-43), state corrections, Eq. (3-45), and a posteriori covariance matrix, Eq. (3-47), were calculated. Table 4-5 was constructed which distinctly defines each expression "Correction" portion of the filtering process.

Table 4-5: "Correction" Equations (Method I)

\begin{tabular}{|c|c|c|c|c|}
\hline & Nomenclature & Symbol & $\begin{array}{l}\text { Matrix } \\
\text { Size }\end{array}$ & Values \\
\hline \multirow{4}{*}{$\begin{array}{l}\text { 1) Compute the Kalman Gain } \\
K_{k}=P_{k+1}^{-} H_{k}^{T}\left[H_{k} P_{k+1}^{-} H_{k}^{T}+R_{k}\right]^{-1}\end{array}$} & $\begin{array}{c}\text { Error } \\
\text { Covariance } \\
\text { Matrix }\end{array}$ & $P_{k+1}^{-}$ & $6 x 6$ & {$\left[\operatorname{diag}\left(X_{6 x 6}\right)\right]$} \\
\hline & $\begin{array}{l}\text { Measurement } \\
\text { Covariance } \\
\text { Matrix }\end{array}$ & $R_{k}$ & $6 \times 6$ & {$\left[\begin{array}{cc}\operatorname{diag}\left(\sigma_{r, 3 \times 3}^{2}\right) & 0_{3 \times 3} \\
0_{3 \times 3} & \operatorname{diag}\left(\sigma_{v, 3 \times 3}^{2}\right)\end{array}\right]$} \\
\hline & Kalman Gain & $K_{k}$ & $6 \times 6$ & {$\left[\operatorname{diag}\left(X_{6 x 6}\right)\right]$} \\
\hline & $\begin{array}{l}\text { Observation } \\
\text { Matrix }\end{array}$ & $H_{k}$ & $6 x 6$ & {$\left[\begin{array}{ll}I_{3 \times 3} & 0_{3 x 3} \\
0_{3 x 3} & I_{3 x 3}\end{array}\right]$} \\
\hline \multirow{3}{*}{$\begin{array}{c}\text { 2) Update Estimate with } \\
\text { Measurement " } z \text { " } \\
\hat{x}_{k}=\hat{x}_{k}^{-}+K_{k}\left(z_{k}-H_{k} \hat{x}_{k}^{-}\right)\end{array}$} & System States & $\hat{x}_{k+1}^{-}, \hat{x}_{k}$ & $6 \times 1$ & {$\left[\begin{array}{l}x_{k, 3 x 1} \\
v_{k, 3 x 1}\end{array}\right]$} \\
\hline & $\begin{array}{c}\text { GPS } \\
\text { Measurement } \\
\text { Value } \\
\end{array}$ & $z_{k}$ & $6 \times 1$ & {$\left[\begin{array}{l}x_{k, 3 x 1} \\
v_{k, 3 x 1}\end{array}\right]$} \\
\hline & $\begin{array}{l}\text { Observation } \\
\text { Matrix }\end{array}$ & $H_{k}$ & $6 \times 6$ & {$\left[\begin{array}{ll}I_{3 \times 3} & 0_{3 x 3} \\
0_{3 \times 3} & I_{3 \times 3}\end{array}\right]$} \\
\hline $\begin{array}{l}\text { 3) Update the Error Covariance: } \\
P_{k+1}^{+}=\left(I_{6 x 6}-K_{k} H_{k}\right) P_{k+1}^{-}\end{array}$ & $\begin{array}{c}\text { Error } \\
\text { Covariance } \\
\text { Matrix }\end{array}$ & $P_{k+1}^{-}, P_{k}^{+}$ & $6 \times 6$ & {$\left[\operatorname{diag}\left(X_{6 x 6}\right)\right]$} \\
\hline
\end{tabular}




\begin{tabular}{|c|c|c|c|c|}
\hline & Kalman Gain & $K_{k}$ & $6 x 6$ & {$\left[X_{6 x 6}\right]$} \\
\cline { 2 - 5 } & $\begin{array}{c}\text { Observation } \\
\text { Matrix }\end{array}$ & $H_{k}$ & $6 x 6$ & {$\left[\begin{array}{cc}I_{3 \times 3} & 0_{3 \times 3} \\
0_{3 x 3} & I_{3 \times 3}\end{array}\right]$} \\
\hline
\end{tabular}

Further describing the previous table, the state correction contains the observation vector $(z)$, predicted observation vector $\left(H_{k} \hat{x}_{k}^{-}\right)$, and the Kalman gain matrix $(\mathrm{K})$. The observation vector was a $6 \times 1$ matrix that is composed of the GPS values for position and velocity while the $6 x 1$ predicted observation vector was composed of the INS values for the position and velocity. The observation vector and predicted observation vector were then differenced to make up the residual in Eq. (3-45), which then gets multiplied by the Kalman gain and summed with the state estimate to produce the filtered results. The updated state vector was then fed back into the INS computation to predict the next increment.

At this point, all of the parameters, inputs, and expressions needed for the filtering process have been well defined and the simulation was coded in Matlab ${ }^{\circledR}$. As described in section 4.2.2, the IMU and GPS data is obtained at 40 and $20 \mathrm{~Hz}$ respectively, meaning that filtering will occur every other reading obtained from the IMU, which is illustrated graphically in Figure 4.9. 


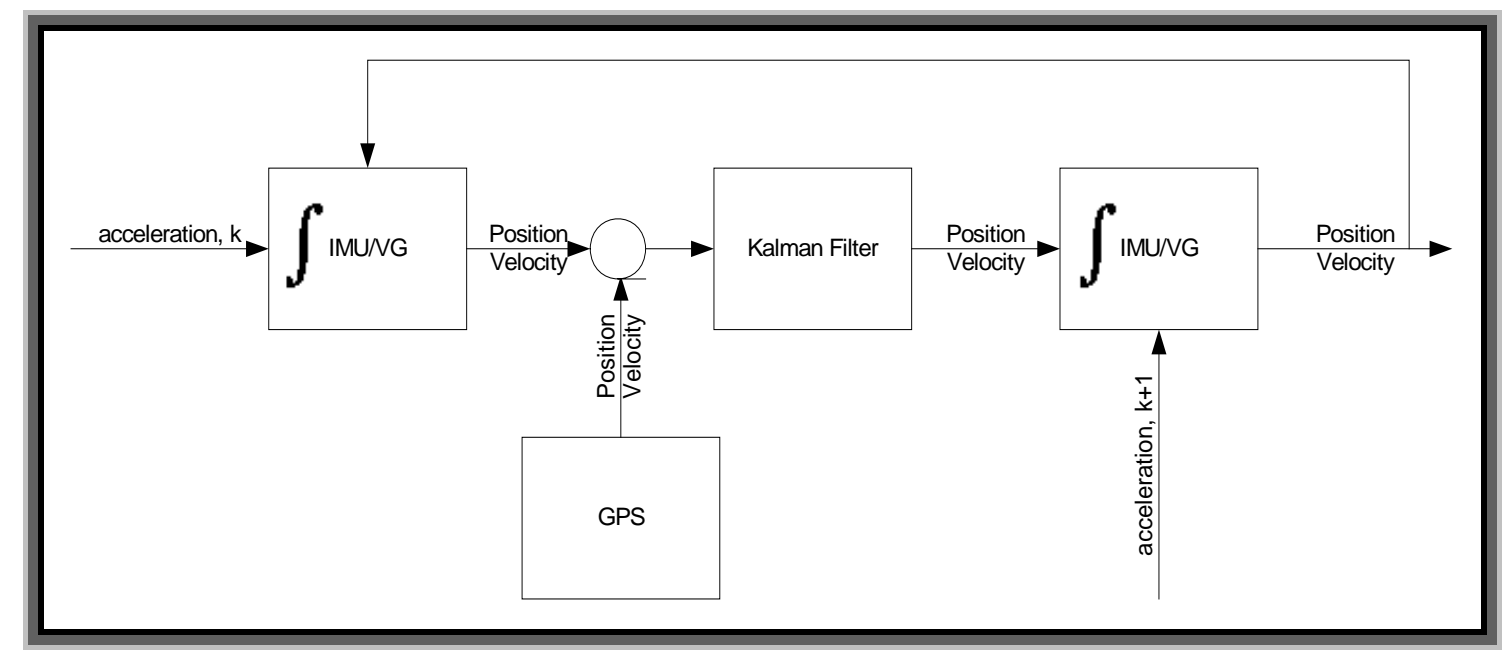

Figure 4.9: Filter Process Sequence

\subsubsection{Fusion of IMU/GPS Data Using GPS Attitude Estimates}

This second method fuses the GPS position and velocity/GPS estimated attitudes with the INS position, velocity, and attitude. There are two main differences from the previous method, which now entail the calculation of the non-linear kinematic equations to determine the aircraft attitude, which also forces the need for the EKF and the estimation of the GPS attitudes discussed in Section 3.4. This is the first implementation in which the vertical gyro is eliminated and the navigation system relies solely on the extended Kalman filter to correct for the attitude drift.

In addition to the first two functions conducted in the first method, this second method also entails:

1) Data Preprocessing (From Method I)

2) INS Calculations (From Method I)

3) Attitude Integration and Linearization

4) GPS Attitude Estimation

5) Extended Kalman Filter Implementation.

Most of the data preprocessing and parameter initialization remains consistent from the first method; the major differences for the implementation are the addition of the nonlinear calculations of the attitudes in the EKF, the estimation of the model/input 
covariance matrix $(Q)$, and the addition of the GPS attitude variance values to the measurement covariance matrix which is depicted graphically in Figure 4.10.

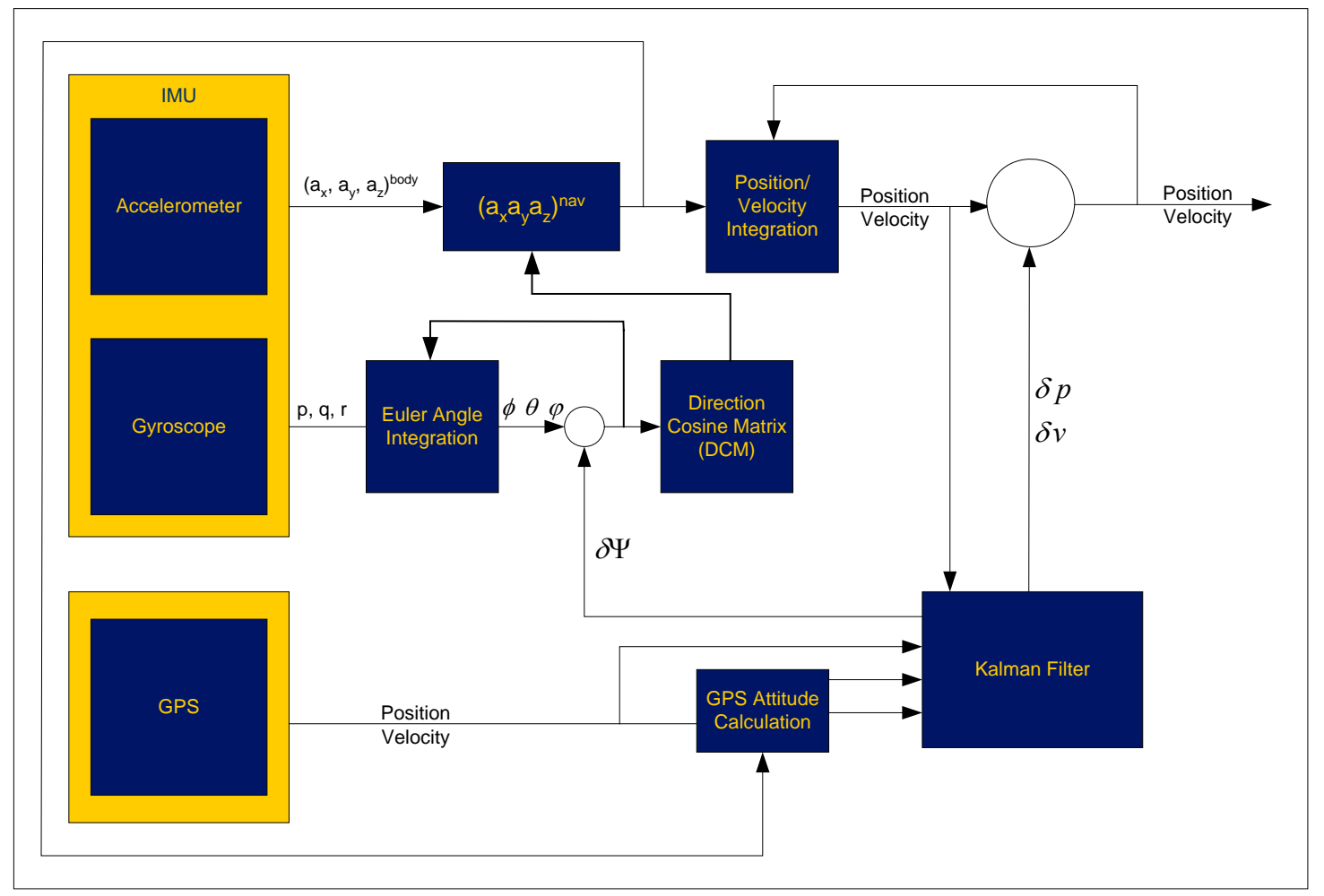

Figure 4.10: Block Diagram for the GPS Aided INS System

With the addition of the GPS attitude in the observation vector, $z$, the measurement covariance matrix, $R$, gets expanded from the $6 x 6$ diagonal matrix composed of the position and velocity covariance to a $9 \times 9$ diagonal matrix composed of the position, velocity, and attitude.

$$
R=\left[\begin{array}{ccccccccc}
\sigma_{x_{\text {Norh }}}^{2} & 0 & 0 & 0 & 0 & 0 & 0 & 0 & 0 \\
0 & \sigma_{y_{\text {East }}}^{2} & 0 & 0 & 0 & 0 & 0 & 0 & 0 \\
0 & 0 & \sigma_{z_{\text {Down }}}^{2} & 0 & 0 & 0 & 0 & 0 & 0 \\
0 & 0 & 0 & \sigma_{u_{\text {Norh }}}^{2} & 0 & 0 & 0 & 0 & 0 \\
0 & 0 & 0 & 0 & \sigma_{v_{\text {East }}}^{2} & 0 & 0 & 0 & 0 \\
0 & 0 & 0 & 0 & 0 & \sigma_{w_{\text {Down }}}^{2} & 0 & 0 & 0 \\
0 & 0 & 0 & 0 & 0 & 0 & \sigma_{\phi}^{2} & 0 & 0 \\
0 & 0 & 0 & 0 & 0 & 0 & 0 & \sigma_{\theta}^{2} & 0 \\
0 & 0 & 0 & 0 & 0 & 0 & 0 & 0 & \sigma_{\varphi}^{2}
\end{array}\right]=
$$




$$
\left[\begin{array}{ccccccccc}
0.0022 & 0 & 0 & 0 & 0 & 0 & 0 & 0 & 0 \\
0 & 0.0017 & 0 & 0 & 0 & 0 & 0 & 0 & 0 \\
0 & 0 & 0.0046 & 0 & 0 & 0 & 0 & 0 & 0 \\
0 & 0 & 0 & 2.0988 \mathrm{e}^{-4} & 0 & 0 & 0 & 0 & 0 \\
0 & 0 & 0 & 0 & 2.0988 \mathrm{e}^{-4} & 0 & 0 & 0 & 0 \\
0 & 0 & 0 & 0 & 0 & 5.3837 \mathrm{e}^{-4} & 0 & 0 & 0 \\
0 & 0 & 0 & 0 & 0 & 0 & 0.0019 & 0 & 0 \\
0 & 0 & 0 & 0 & 0 & 0 & 0 & 0.0023 & 0 \\
0 & 0 & 0 & 0 & 0 & 0 & 0 & 0 & 0
\end{array}\right]
$$

The process for estimating the model/input covariance matrix was conducted the same way as to determine the values for measurement covariance matrix. For the model/input covariance matrix, the variance of the IMU raw accelerometer and angular rate data is used for the diagonal as seen below:

$$
Q=\left[\begin{array}{ccccccc}
0_{3 \times 3} & 0_{1 \times 3} & 0_{1 \times 3} & 0_{1 \times 3} & 0_{1 \times 3} & 0_{1 \times 3} & 0_{1 \times 3} \\
0_{3 \times 1} & \sigma_{a_{x}}^{2} & 0 & 0 & 0 & 0 & 0 \\
0_{3 \times 1} & 0 & \sigma_{a_{y}}^{2} & 0 & 0 & 0 & 0 \\
0_{3 \times 1} & 0 & 0 & \sigma_{a_{z}}^{2} & 0 & 0 & 0 \\
0_{3 \times 1} & 0 & 0 & 0 & \sigma_{p}^{2} & 0 & 0 \\
0_{3 \times 1} & 0 & 0 & 0 & 0 & \sigma_{q}^{2} & 0 \\
0_{3 \times 1} & 0 & 0 & 0 & 0 & 0 & \sigma_{r}^{2}
\end{array}\right]=\left[\begin{array}{ccccccc}
0_{3 \times 3} & 0_{1 \times 3} & 0_{1 \times 3} & 0_{1 \times 3} & 0_{1 \times 3} & 0_{1 \times 3} & 0_{1 \times 3} \\
0_{3 \times 1} & .004 & 0 & 0 & 0 & 0 & 0 \\
0_{3 x 1} & 0 & .0037 & 0 & 0 & 0 & 0 \\
0_{3 \times 1} & 0 & 0 & .0046 & 0 & 0 & 0 \\
0_{3 \times 1} & 0 & 0 & 0 & 1.843 \mathrm{e}^{-8} & 0 & 0 \\
0_{3 \times 1} & 0 & 0 & 0 & 0 & 1.289 \mathrm{e}^{-8} & 0 \\
0_{3 \times 1} & 0 & 0 & 0 & 0 & 0 & 1.293 \mathrm{e}^{-8}
\end{array}\right]
$$

The addition of the INS attitude creates two major changes to the software algorithm; first, to project the state ahead, the nonlinear aircraft kinematic equations are used, while secondly, the aircraft kinematic equation's Jacobian matrix of partial derivates from Equation (3-19) is inserted into the state transition matrix for use in the calculation of the a priori error covariance matrix. Table 4-6 shows the "Prediction" calculations used for this method in which the determination of the state vector and Jacobian matrix was discussed in detail in Section 3.3.2. 
Table 4-6: "Prediction" Equations (Method II)

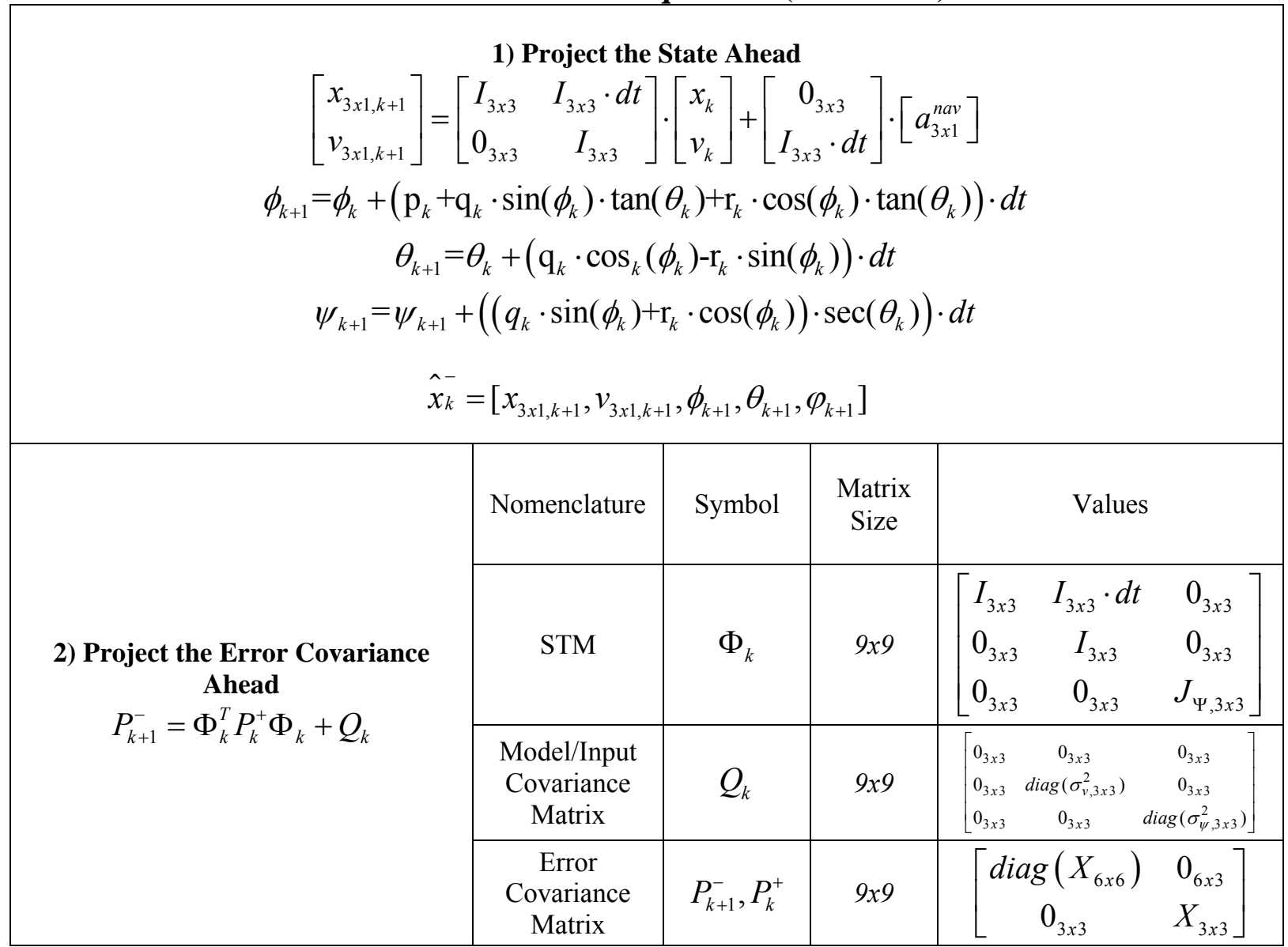

The resulting "Correction" calculations seen in Table 4-7 are similar to that of the previous method with the exception that the GPS attitude was added to the GPS observation vector, $z$, and the change in the size of each matrix to accommodate the additional three components of the attitude. The new observation vector was a $9 x 1$ matrix that is composed of the GPS values for position, velocity, and attitude while the $9 x 1$ predicted observation vector was composed of the INS values for the position, velocity, and attitude. The following table shows the "Correction" calculations including a detailed description.

Table 4-7: "Correction" Equations (Method II)

\begin{tabular}{|l|c|c|c|c|}
\hline & Nomenclature & Symbol & $\begin{array}{c}\text { Matrix } \\
\text { Size }\end{array}$ & Values \\
\hline 1) Compute the Kalman Gain & $\begin{array}{c}\text { Observation } \\
\text { Matrix }\end{array}$ & $H_{k}$ & $9 x 9$ & {$\left[I_{9 x 9}\right]$} \\
\hline
\end{tabular}




\begin{tabular}{|c|c|c|c|c|c|}
\hline \multirow[t]{4}{*}{$K_{k}=P_{k+1}^{-} H_{k}^{T}\left[H_{k} P_{k+1}^{-} H_{k}^{T}+R_{k}\right]^{-1}$} & $\begin{array}{l}\text { Error } \\
\text { Covariance } \\
\text { Matrix }\end{array}$ & $P_{k+1}^{-}$ & $9 \times 9$ & \multicolumn{2}{|c|}{$\left[\begin{array}{cc}\operatorname{diag}\left(X_{6 \times 6}\right) & 0_{6 x 3} \\
0_{3 \times 3} & X_{3 x 3}\end{array}\right]$} \\
\hline & $\begin{array}{l}\text { Measurement } \\
\text { Covariance } \\
\text { Matrix }\end{array}$ & $R_{k}$ & $9 \times 9$ & {$\left[\begin{array}{cc}\operatorname{diag}\left(\sigma_{r, 3 \times 3}^{2}\right) & 0_{3 x 3} \\
0_{3 \times 3} & \operatorname{diag}\left(\sigma_{v, 3 \times 3}^{2}\right) \\
0_{3 \times 3} & 0_{3 x 3}\end{array}\right]$} & $\left.\begin{array}{c}0_{3 \times 3} \\
0_{3 \times 3} \\
\operatorname{diag}\left(\sigma_{\Psi, 3 \times 3}^{2}\right)\end{array}\right]$ \\
\hline & Kalman Gain & $K_{k}$ & $9 \times 9$ & {$\left[X_{9 x 9}\right]$} & \\
\hline & System States & $\hat{x}_{k+1}^{-}, \hat{x}_{k}$ & $9 \times 1$ & {$\left[\begin{array}{l}x_{k, 3 x 1} \\
v_{k, 3 x 1} \\
\Psi_{k, 3 x 1}\end{array}\right]$} & \\
\hline \multirow{3}{*}{ 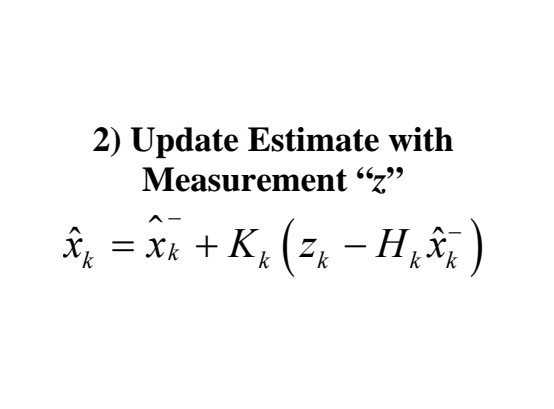 } & $\begin{array}{l}\text { GPS } \\
\text { Observation } \\
\text { Vector }\end{array}$ & $z_{k}$ & $9 \times 1$ & {$\left[\begin{array}{c}x_{k, 3 x 1} \\
v_{k, 3 x 1} \\
\Psi_{k, 3 x 1}\end{array}\right]$} & \\
\hline & $\begin{array}{l}\text { Observation } \\
\text { Matrix }\end{array}$ & $H_{k}$ & $9 \times 9$ & {$\left[I_{9 x 9}\right]$} & \\
\hline & $\begin{array}{l}\text { Error } \\
\text { Covariance } \\
\text { Matrix }\end{array}$ & $P_{k+1}^{-}, P_{k}^{+}$ & $9 \times 9$ & {$\left[\begin{array}{c}\operatorname{diag}\left(X_{6 \times 6}\right) \\
0_{3 \times 3}\end{array}\right.$} & $\left.\begin{array}{l}0_{6 \times 3} \\
X_{3 \times 3}\end{array}\right]$ \\
\hline \multirow{3}{*}{$\begin{array}{l}\text { 3) Update the Error Covariance } \\
P_{k+1}^{+}=\left(I_{6 x 6}-K_{k} H_{k}\right) P_{k+1}^{-}\end{array}$} & Kalman Gain & $K_{k}$ & $9 \times 9$ & {$\left[X_{9 x 9}\right]$} & \\
\hline & $\begin{array}{c}\text { Observation } \\
\text { Matrix }\end{array}$ & $H_{k}$ & $9 \times 9$ & {$\left[I_{9 x 9}\right]$} & \\
\hline & $\begin{array}{l}\text { Error } \\
\text { Covariance } \\
\text { Matrix }\end{array}$ & $P_{k+1}^{-}$ & $9 \times 9$ & {$\left[\begin{array}{c}\operatorname{diag}\left(X_{6 \times 6}\right) \\
0_{3 \times 3}\end{array}\right.$} & $\left.\begin{array}{l}0_{6 \times 3} \\
X_{3 \times 3}\end{array}\right]$ \\
\hline
\end{tabular}

At this point, all of the parameters, inputs, and expressions needed for the filtering process have been well defined and the simulation was coded in Matlab ${ }^{\circledR}$. As described in the previous section, the IMU and GPS data was again obtained at 40 and $20 \mathrm{~Hz}$ respectively, meaning that the filtering occurred every other measurement obtained from the IMU.

\subsubsection{Fusion of IMU/GPS data}

The third method directly corrects the position and velocity within the Kalman filter algorithm, and indirectly corrects the attitudes since they directly map to the position and velocity through the direction cosine matrix. This method compensates for the Euler angle errors by incorporating the DCM into the system matrix instead of converting the acceleration to the local axis prior to the filtering process, which is the only link between the GPS data and the IMU angular rate data. Without having the DCM 
in the system matrix or a measurement value for the residual, no compensation can occur within the filter. With this said, the third method required the following changes to be made in the formulation of the simulation algorithm:

1. Data Preprocessing (From Method I \& II)

2. INS Calculations (From Method II)

3. Attitude Integration and Linearization (From Method II)

4. DCM Linearization

5. Extended Kalman Filter Implementation

Elements from the data preprocessing and parameter initialization remain consistent from the first two methods, although with the removal of the GPS attitude estimates, the measurement covariance matrix, $R$, resort back to the matrix used in method $\mathrm{I}$, while the system/input covariance matrix, $Q$, is the same as method II.

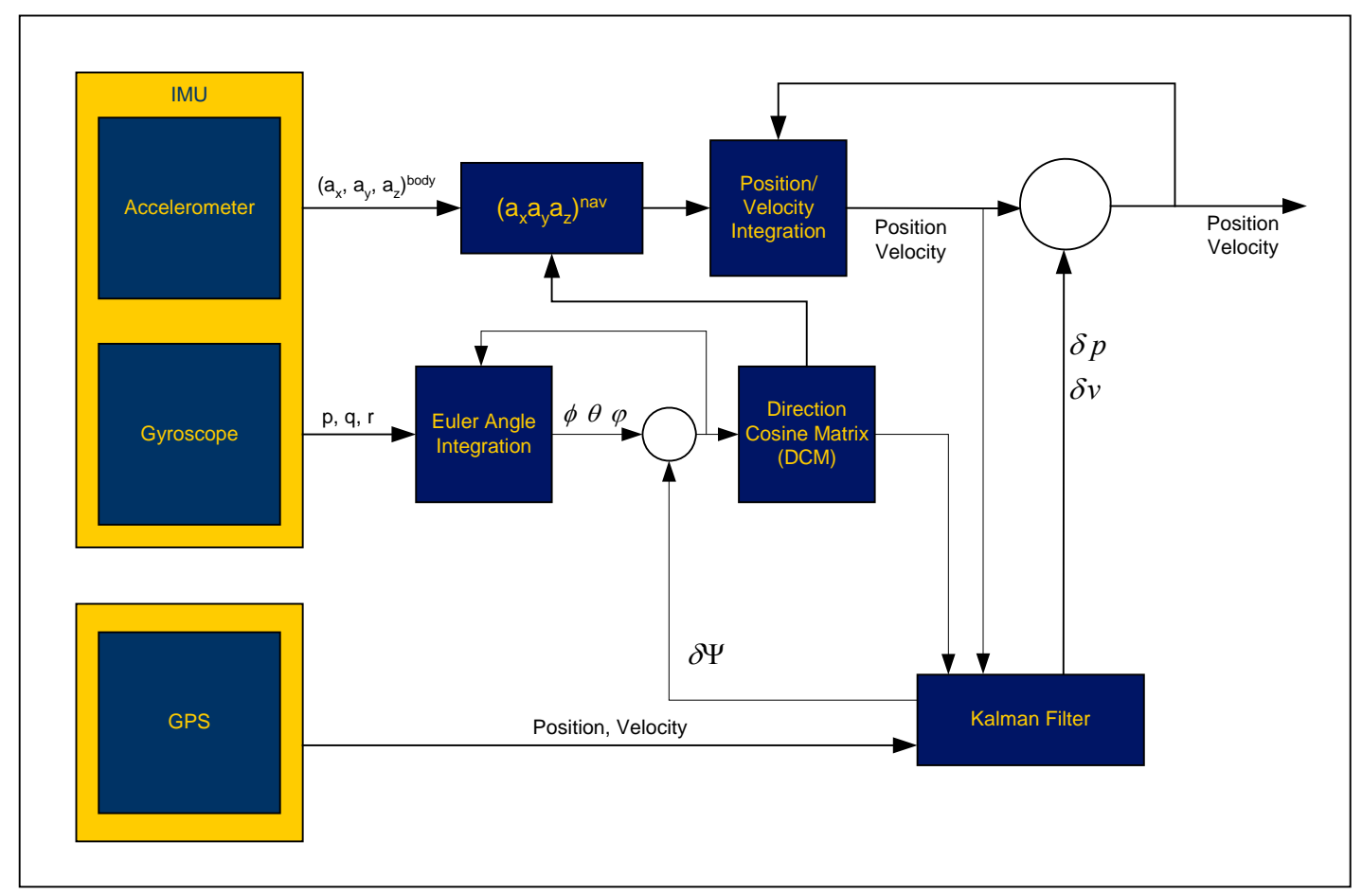

Figure 4.11: Block Diagram for the GPS/INS System (Method III)

To further elaborate on the introduction, if the STM is only made up of the position, velocity, and kinematic equations, the error covariance matrix will take on the form depicted at point $A$ in Figure 4.12 below. This value is then input into the Kalman 
gain calculation in which the gain turns out to be a $9 \times 6$ matrix with the bottom three rows being zeros, as seen at point $B$. These bottom three rows of the gain matrix make up the gain associated with the attitude, causing there to be no correction to the estimated attitude states, resulting in the final output at point $C$. (NOTE: Positions within Figure 4.12 containing the character ' $\mathrm{X}$ ' represent any real number.)

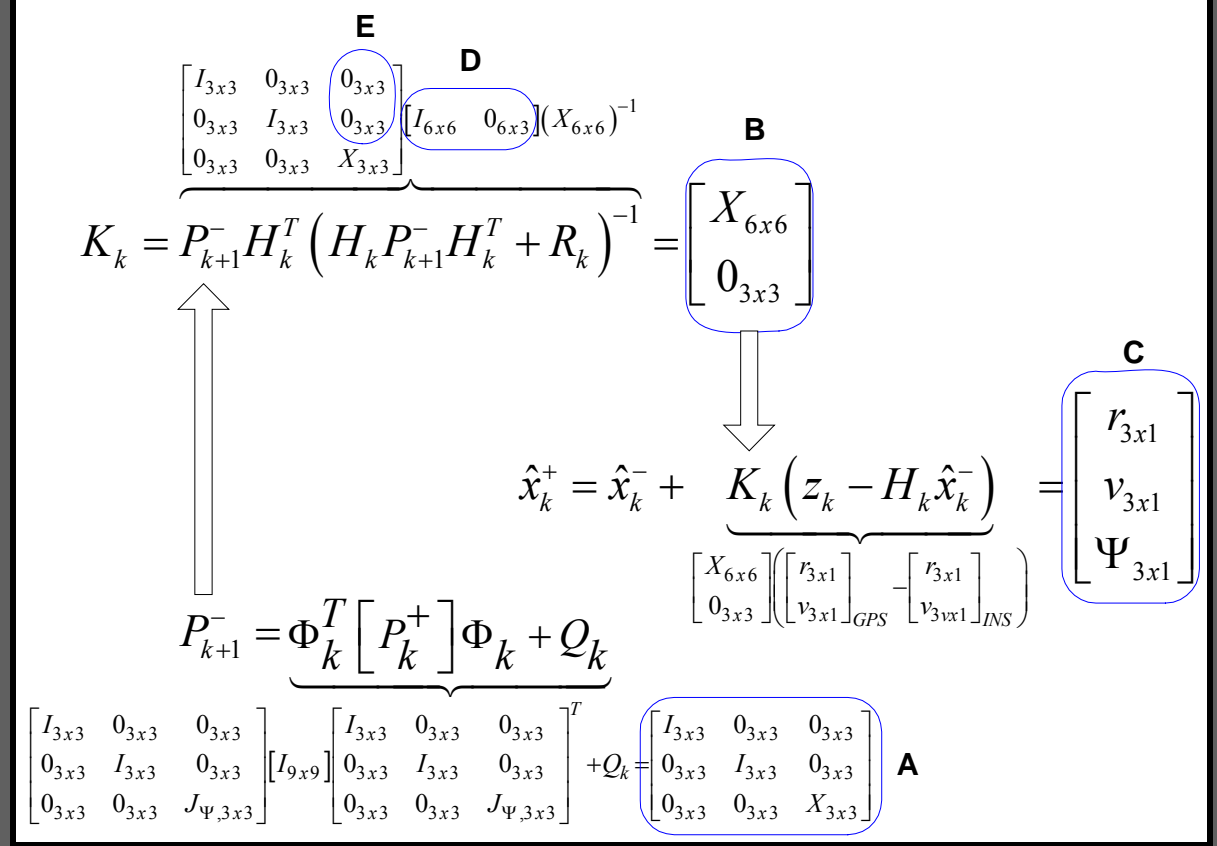

Figure 4.12: Kalman Process Diagram

With further inspection of Figure 4.12, to be able to obtain a gain within the bottom three rows at $B$, it is obvious that there are only two options. The first is to have an additional measurement value (e.g. GPS attitude estimate as used for the previous section) so that the observation matrix, $(H$, point $D)$, becomes a $9 x 9$ matrix, allowing the Kalman gain to correct for all of the states.

The second option is to generate nonzero values for the first two rows of the third column in the state transition matrix, which would then allow nonzero values to be obtained in the error covariance matrix (point $E$ ). This is achieved by placing the DCM directly into the second row, third column of the STM so that the error associated with the rotation from the body axis to the local tangent frame can be estimated by the gain. Since the calculation of the DCM is nonlinear, a Taylor series approximation is now required for the velocity states in a similar fashion to the linearization of the aircraft 
kinematic equations. Only the Jacobian approximation for the system matrix is displayed due to the fact that the linearized input matrix is not utilized in the filtering process. Also keep in mind that the error associated with the hot's is neglected.

$$
\begin{gathered}
J(\phi, \theta, \varphi)=\left[\begin{array}{ccc}
\frac{\delta f(\dot{\theta})}{\delta \phi} & \frac{\delta f(\dot{\phi})}{\delta \theta} & \frac{\delta f(\dot{\varphi})}{\delta \varphi} \\
\frac{\delta f(\dot{\theta})}{\delta \phi} & \frac{\delta f(\dot{\phi})}{\delta \theta} & \frac{\delta f(\dot{\varphi})}{\delta \varphi} \\
\frac{\delta f(\dot{\theta})}{\delta \phi} & \frac{\delta f(\dot{\phi})}{\delta \theta} & \frac{\delta f(\dot{\varphi})}{\delta \varphi}
\end{array}\right]= \\
{\left[\begin{array}{ccc}
\mathrm{q} \cdot \cos (\phi) \cdot \tan (\theta)-\mathrm{r} \cdot \sin (\phi) \cdot \tan (\theta) & q \cdot \sin (\phi) \cdot \sec ^{2}(\theta)+\mathrm{r} \cdot \cos (\phi) \cdot \sec ^{2}(\theta) & 0 \\
-q \cdot \sin (\phi)-\mathrm{r} \cdot \cos (\phi) & 0 & 0 \\
(q \cdot \cos (\phi)-\mathrm{r} \cdot \sin (\phi)) \cdot \sec (\theta) & (q \cdot \sin (\phi)+\mathrm{r} \cdot \cos (\phi)) \cdot \sec (\theta) \cdot \tan (\theta) & 0
\end{array}\right]}
\end{gathered}
$$

After substituting the DCM Jacobian matrix into the state transition matrix the error covariance matrix takes in the form seen at point $A$ in Figure 4.13. This in turn allows for the attitude error to be estimated in the bottom three rows of the Kalman gain as seen at point $B$.

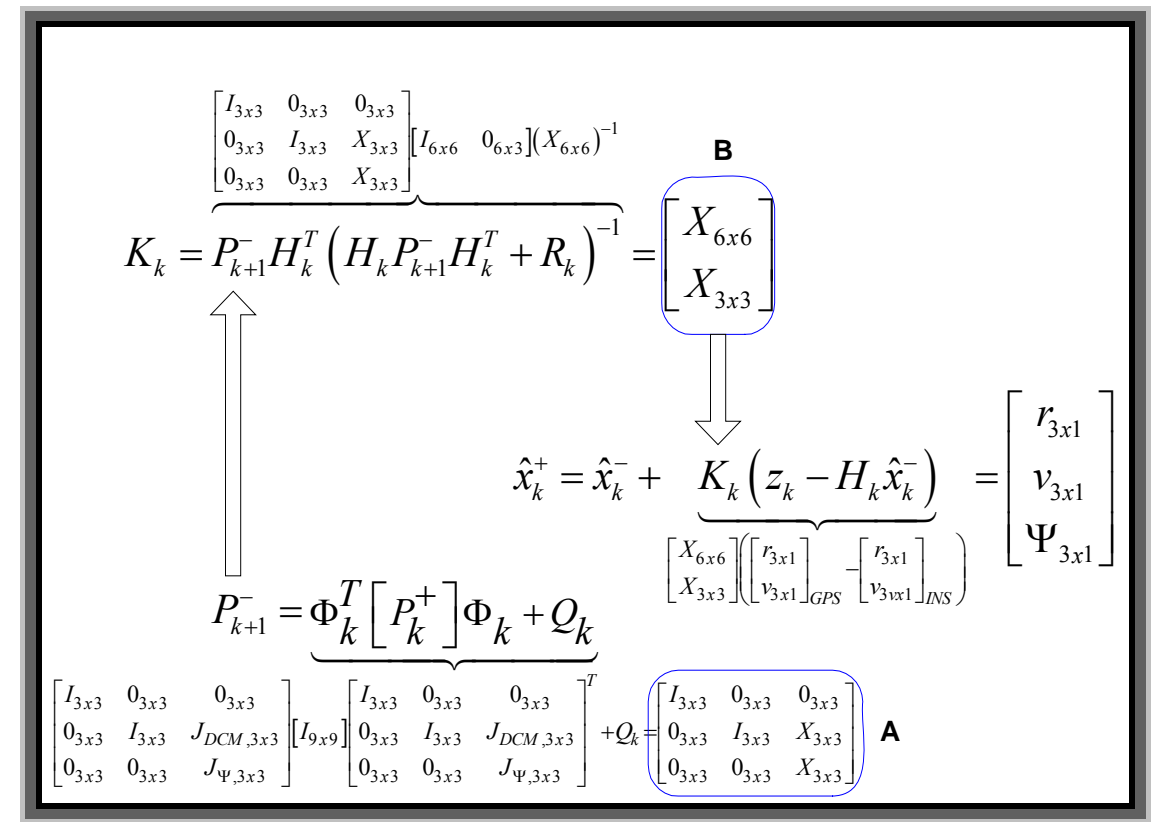

Figure 4.13: Kalman Process Diagram II 
The "prediction" equations and descriptions shown in Table 4-8 are very similar to that of the method II with the exception of the addition of the Jacobian matrix to the state transition matrix.

Table 4-8: "Prediction" Equations (Method III)

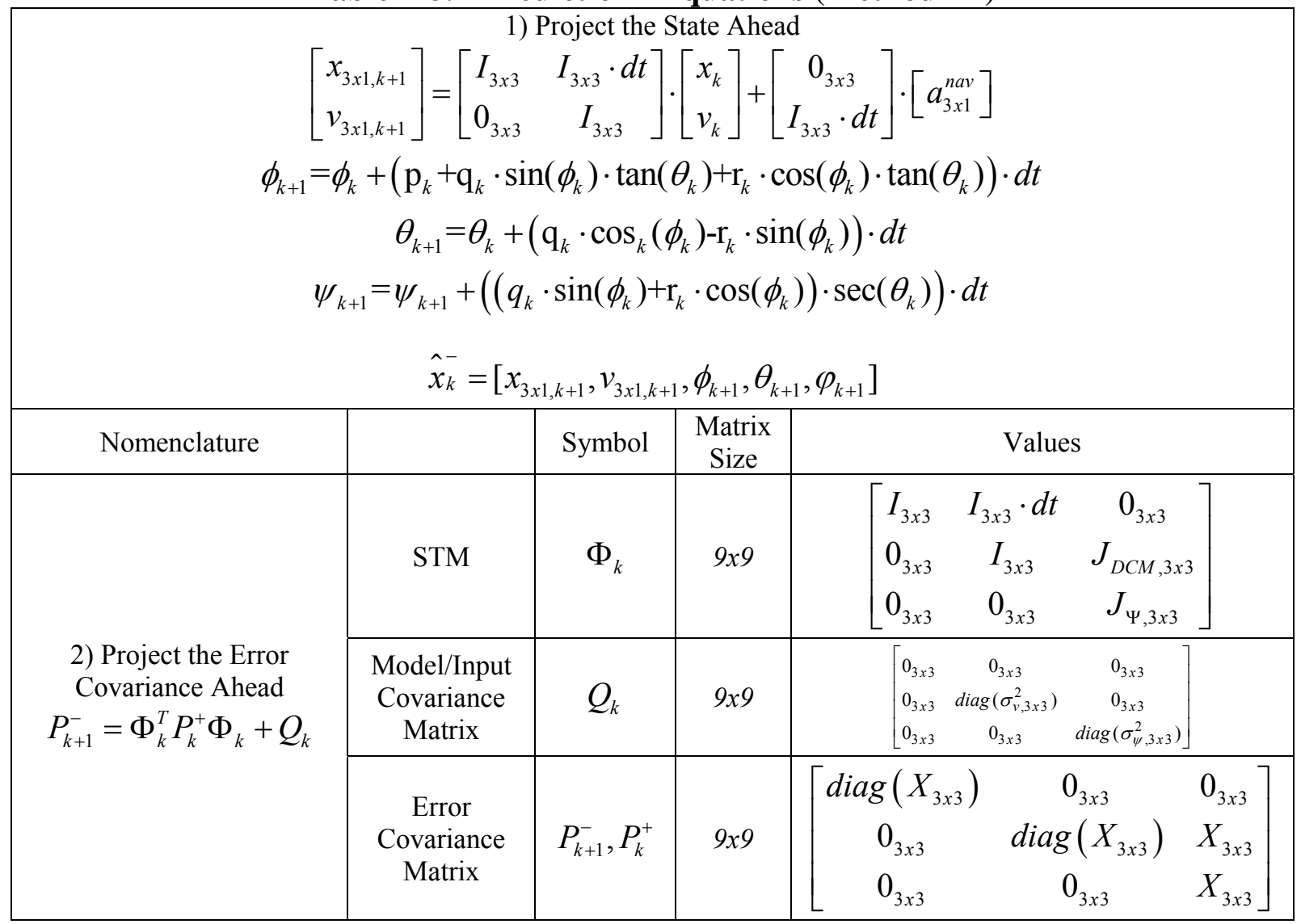

The alterations to the "correction" calculations involved the removal of the GPS estimated attitude values from the GPS measurement value, $z$, and the measurement covariance matrix was reverted back to the $6 x 6$ matrix used in method I. The "correction" calculations and descriptions can be seen in the following table.

Table 4-9: "Correction" Equations (Method III)

\begin{tabular}{|c|c|c|c|cc|}
\hline $\begin{array}{c}\text { 1) Compute the Kalman Gain } \\
K_{k}=P_{k+1}^{-} H_{k}^{T}\left[H_{k} P_{k+1}^{-} H_{k}^{T}+R_{k}\right]^{-1}\end{array}$ & $\begin{array}{c}\text { Observation } \\
\text { Matrix }\end{array}$ & $H_{k}$ & $6 x 9$ & {$\left[\begin{array}{lll}I_{3 \times 3} & 0_{3 \times 3} & 0_{3 \times 3} \\
0_{3 \times 3} & I_{3 \times 3} & 0_{3 \times 3}\end{array}\right]$} \\
\hline & $\begin{array}{c}\text { Error } \\
\text { Covariance } \\
\text { Matrix }\end{array}$ & $P_{k+1}^{-}$ & $9 \times 9$ & & {$\left[\begin{array}{ccc}\operatorname{diag}\left(X_{3 \times 3}\right) & 0_{3 \times 3} & 0_{3 \times 3} \\
0_{3 \times 3} & \operatorname{diag}\left(X_{3 \times 3}\right) & X_{3 \times 3} \\
0_{3 \times 3} & 0_{3 \times 3} & X_{3 \times 3}\end{array}\right]$} \\
\hline
\end{tabular}




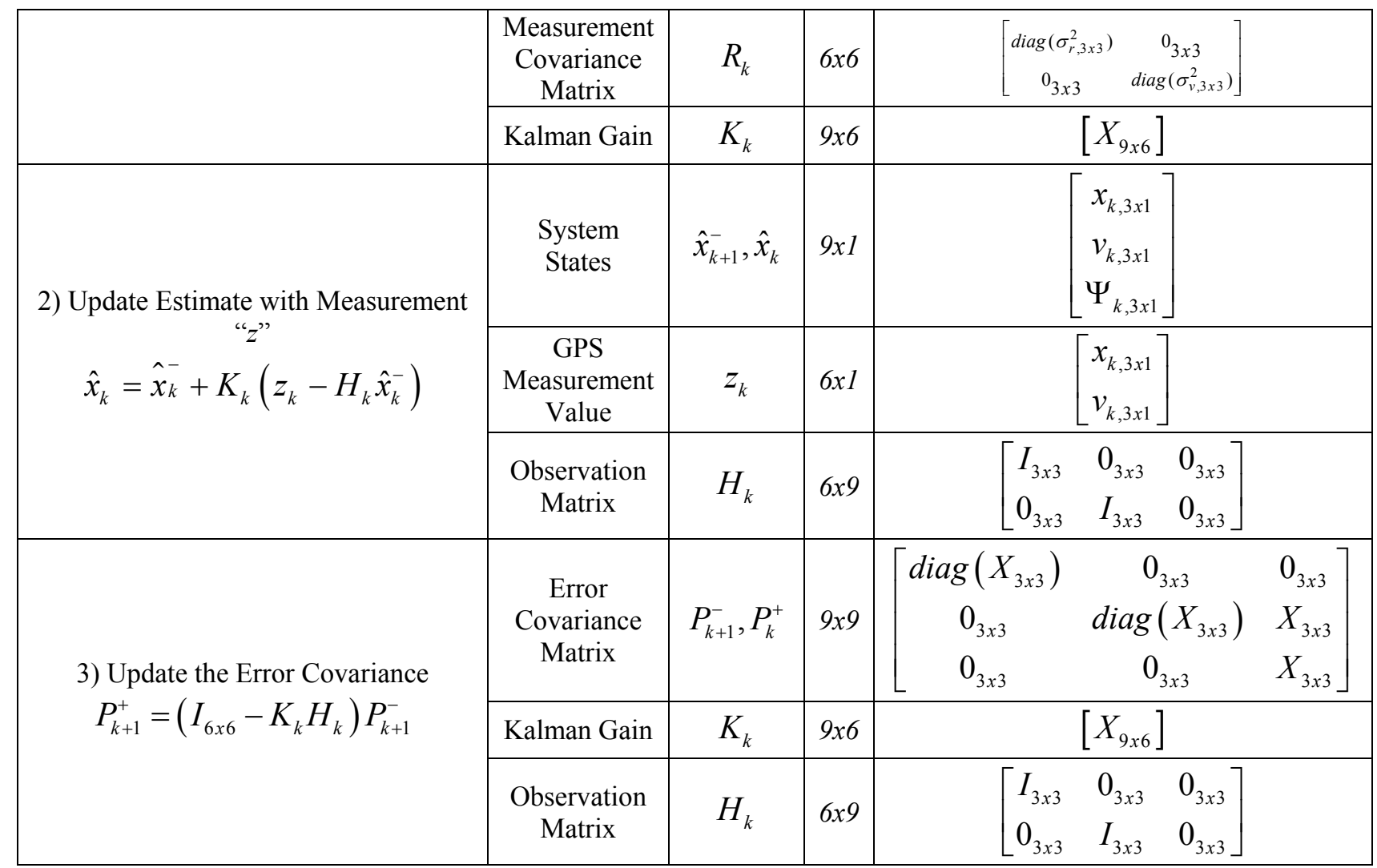

At this point, all of the parameters, inputs, and expressions needed for the filtering process have been well defined and the simulation was coded in Matlab ${ }^{\circledR}$. As described in the previous section, the IMU and GPS data was again obtained at 40 and $20 \mathrm{~Hz}$ respectively, meaning that the filtering occurred every other measurement obtained from the IMU.

\subsubsection{Combination of Method II/Method III}

The fourth and final method for this research project is simply the combination of the second and third methods in which the estimated GPS attitude is used along with the insertion of the linearized DCM into the system model. No additional alterations were required to the model or algorithm, just the manipulation of the Matlab ${ }^{\circledR}$ code to combine the two methods that is depicted graphically in Figure 4.14. 


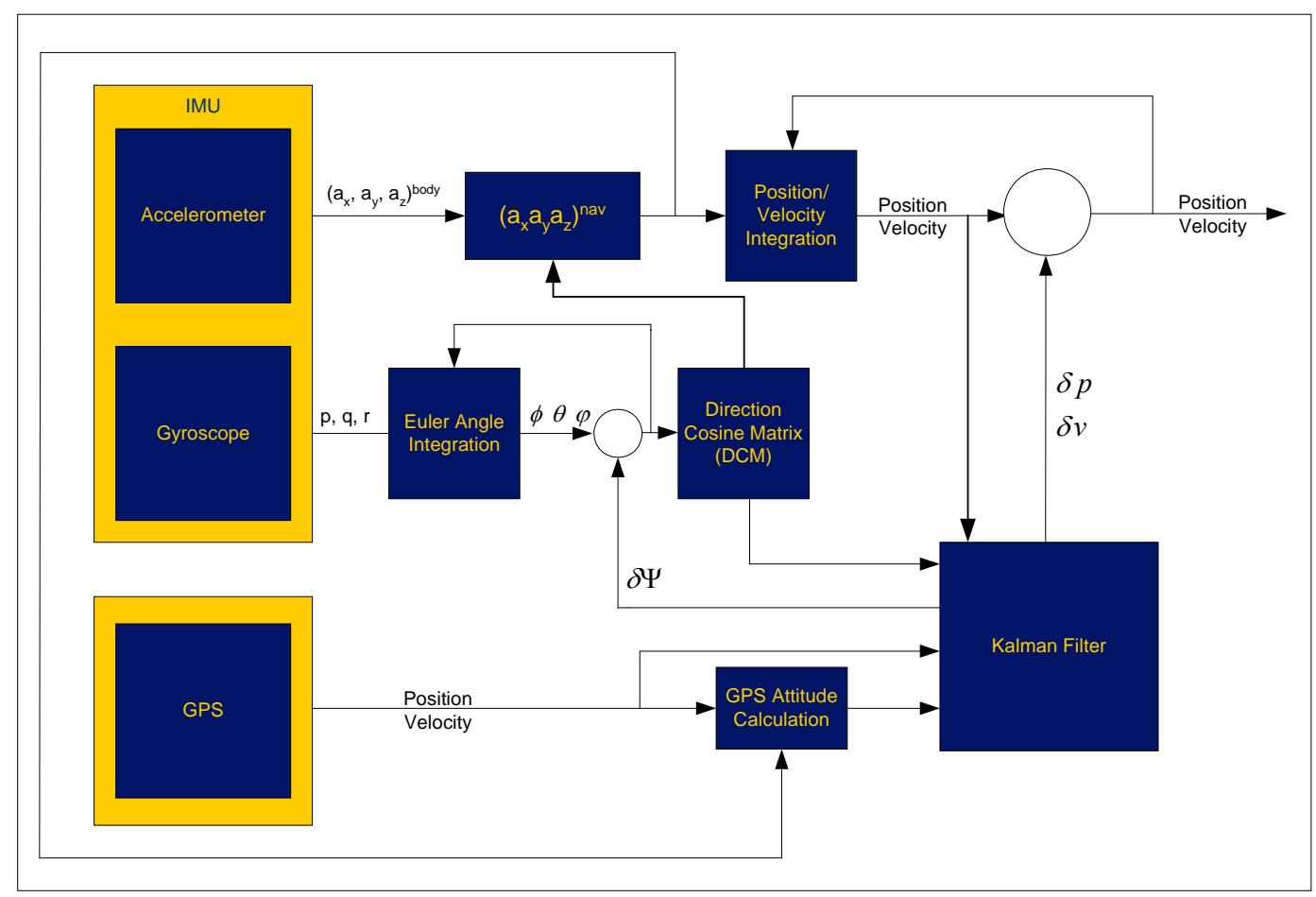

Figure 4.14: Block Diagram GPS Aided Attitude/DCM System

The "prediction" equations and descriptions shown in Table 4-10 are the combination of method II and III's "prediction" equations. As seen from the table, the matrix dimensions are for a full state INS system (nine states), although the position and heading angle could be removed allowing for a five state model. Unlike method II which could be reduced to two states, this method requires at least five states since the DCM is integrated into the system, as discussed in the previous section. 
Table 4-10: "Prediction" Equations (Method IV)

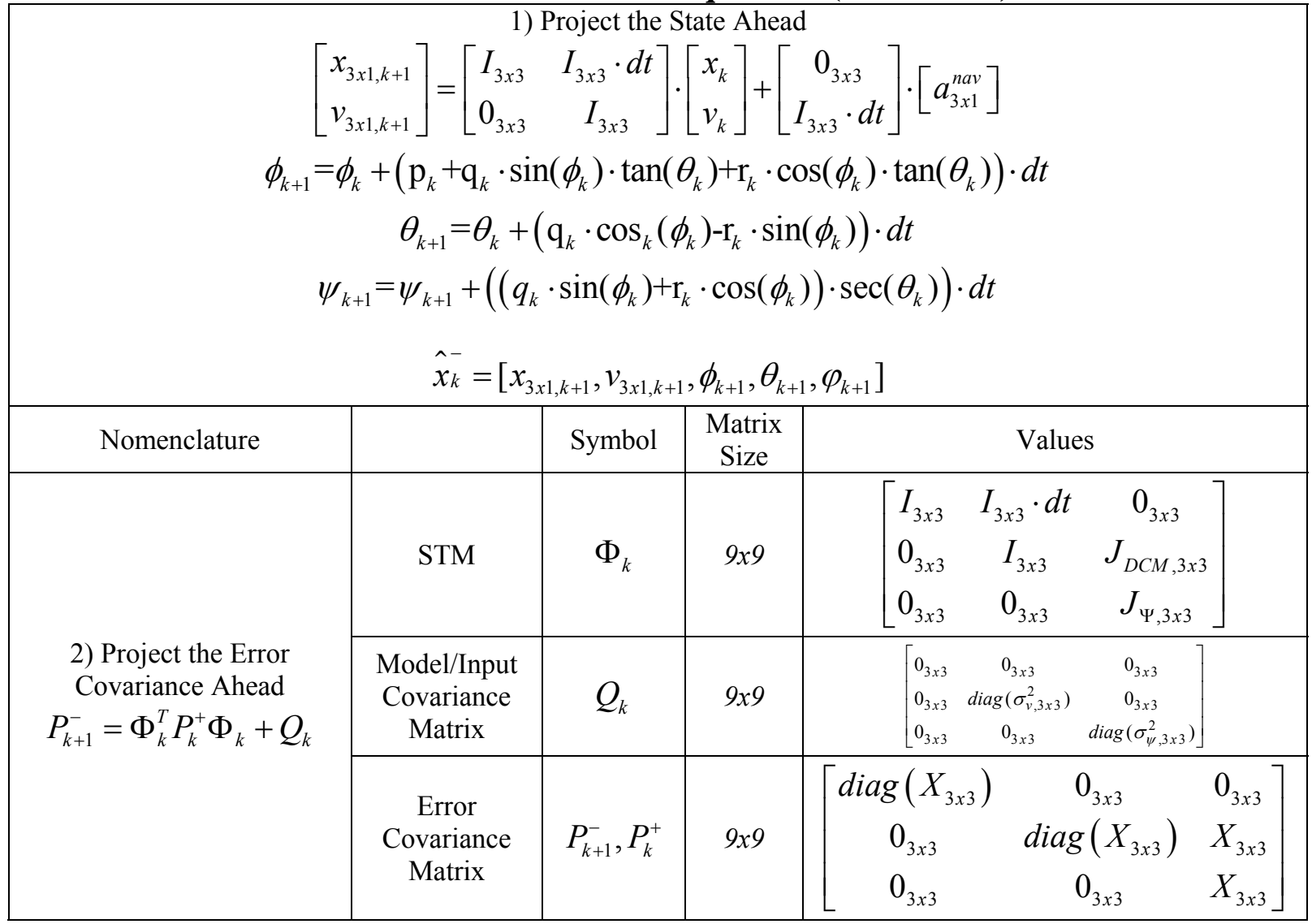

The "correction" calculations also involved the combination of the two methods causing the GPS measurement value, $z$, and the measurement covariance matrix, $R$, to once again to revert back to a $9 \times 1$ and a $9 \times 9$ matrix used in method II. The "correction" calculations and descriptions can be seen in the following table.

Table 4-11: "Correction" Equations (Method IV)

\begin{tabular}{|c|c|c|c|c|c|c|c|}
\hline \multirow{4}{*}{$\begin{array}{c}\text { 1) Compute the Kalman Gain } \\
K_{k}=P_{k+1}^{-} H_{k}^{T}\left[H_{k} P_{k+1}^{-} H_{k}^{T}+R_{k}\right]^{-1}\end{array}$} & $\begin{array}{l}\text { Observation } \\
\text { Matrix }\end{array}$ & $H_{k}$ & $9 \times 9$ & \multicolumn{4}{|c|}{$\left[I_{9 x 9}\right]$} \\
\hline & $\begin{array}{l}\text { Error } \\
\text { Covariance } \\
\text { Matrix }\end{array}$ & $P_{k+1}^{-}$ & $9 \times 9$ & {$\left[\begin{array}{c}\operatorname{diag}\left(X_{3 \times 3}\right) \\
0_{3 x 3} \\
0_{3 x 3}\end{array}\right.$} & $\begin{array}{r}0_{3 x} \\
\operatorname{diag}( \\
0_{3 x}\end{array}$ & $\begin{array}{ll}\left.X_{3 \times 3}\right) & X \\
& X\end{array}$ & $\left.\begin{array}{l}Y_{3 \times 3} \\
X_{3 \times 3} \\
X_{3 \times 3}\end{array}\right]$ \\
\hline & $\begin{array}{l}\text { Measurement } \\
\text { Covariance } \\
\text { Matrix }\end{array}$ & $R_{k}$ & $9 \times 9$ & {$\left[\begin{array}{c}\operatorname{diag}\left(\sigma_{r, 3 \times 3}^{2}\right) \\
0_{3 \times 3} \\
0_{3 \times 3} \\
\end{array}\right.$} & $\begin{array}{c}0_{3 \times 3} \\
\operatorname{diag}\left(\sigma_{v, 3 \times 3}^{2}\right) \\
0_{3 \times 3}\end{array}$ & $\begin{array}{c}0_{3 \times 3} \\
0_{3 \times 3} \\
\operatorname{diag}\left(\sigma_{\Psi, 3 \times 3}^{2}\right)\end{array}$ & \\
\hline & Kalman Gain & $K_{k}$ & $9 \times 9$ & & {$\left[X_{9 x 9}\right]$} & & \\
\hline
\end{tabular}




\begin{tabular}{|c|c|c|c|c|c|c|}
\hline \multirow{3}{*}{$\begin{array}{l}\text { 2) Update Estimate with Measurement } \\
\qquad \hat{x}_{k}=\hat{x}_{k}^{-}+K_{k}\left(z_{k}-H_{k} \hat{x}_{k}^{-}\right)\end{array}$} & $\begin{array}{l}\text { System } \\
\text { States }\end{array}$ & $\hat{x}_{k+1}^{-}, \hat{x}_{k}$ & $9 \times 1$ & & $\left.\begin{array}{l}x_{k, 3 \times 1} \\
v_{k, 3 \times 1} \\
\Psi_{k, 3 x 1}\end{array}\right]$ & \\
\hline & $\begin{array}{l}\text { GPS } \\
\text { Measurement } \\
\text { Value }\end{array}$ & $z_{k}$ & $9 \times 1$ & & $\left.\begin{array}{l}x_{k, 3 \times 1} \\
v_{k, 3 \times 1} \\
\Psi_{k, 3 x 1}\end{array}\right]$ & \\
\hline & $\begin{array}{c}\text { Observation } \\
\text { Matrix }\end{array}$ & $H_{k}$ & $9 \times 9$ & & {$\left[I_{9 x 9}\right]$} & \\
\hline \multirow{3}{*}{$\begin{array}{l}\text { 3) Update the Error Covariance } \\
P_{k+1}^{+}=\left(I_{6 x 6}-K_{k} H_{k}\right) P_{k+1}^{-}\end{array}$} & $\begin{array}{l}\text { Error } \\
\text { Covariance } \\
\text { Matrix }\end{array}$ & $P_{k+1}^{-}, P_{k}^{+}$ & $9 \times 9$ & {$\left[\begin{array}{c}\operatorname{diag}\left(X_{3 \times 3}\right) \\
0_{3 \times 3} \\
0_{3 \times 3} \\
\end{array}\right.$} & $\begin{array}{c}0_{3 \times 3} \\
\operatorname{diag}\left(X_{3 \times 3}\right) \\
0_{3 \times 3} \\
\end{array}$ & $\left.\begin{array}{l}0_{3 \times 3} \\
X_{3 \times 3} \\
X_{3 \times 3}\end{array}\right]$ \\
\hline & Kalman Gain & $K_{k}$ & $9 \times 9$ & \multicolumn{3}{|c|}{$\left[X_{9 x 9}\right]$} \\
\hline & $\begin{array}{c}\text { Observation } \\
\text { Matrix }\end{array}$ & $H_{k}$ & $9 \times 9$ & \multicolumn{3}{|c|}{$\left[I_{9 x 9}\right]$} \\
\hline
\end{tabular}

At this point, all of the parameters, inputs, and expressions needed for the filtering process have been well defined and the simulation was coded in Matlab ${ }^{\circledR}$. 


\section{Chapter 5. Simulation Results and Discussion}

\subsection{Introduction}

This section discusses the simulation results from the fusion of the INS/GPS navigation estimates from the formation flight data. As discussed in the introduction, the simulations were conducted using actual flight data recorded from the WVU formation flight tests, each flights consisted of 900 seconds of flight data including sensor initialization, take-off, flight, and landing within that time frame. This real world data is susceptible to noise and error, as noted earlier, the GPS's main signal deterioration is due to periods of high bank angles, while the IMU obtains additional noise disturbance (e.g., engine vibration, electrical interference) all of which cannot be sufficiently duplicated and simulated within a controlled lab environment. This increases the validity of the results, proving that sensor fusion techniques can achieve a level of performance sufficient for real world UAV control applications.

Each of the four methods were simulated, evaluated, and discussed throughout this section so that it can be seen how the alteration of various parameters can affect the precision and effectiveness of the filter in each of the various implementations and applications while also showing that sensor fusion is an effective way of reducing error within the aircraft components.

The first method discussed in Section 4.2.3.1 improved the position and velocity with the use of the GPS, INS, and Vertical Gyro. While this method does not provide any reduction to the cost, weight, and power consumption of the aircraft, it does however show a significant reduction in the drifting error from the INS system and removes the caused errors in the GPS data.

Simulation results from the second method utilized GPS attitude estimation values in the filter as discussed in Section 4.2.3.2, where the GPS attitude values allowed there to be three measurement values to correct for the three states. With this being the first

implementation of the EKF, it begins to show the power of the Kalman filter by taking two attitude estimates, both with large, but different errors and generates a significant increase in position, velocity, and attitude position. 
The third method used only the position and velocity as the measurement values to correct the position, velocity, and attitude as discussed in Section 4.2.3.3. This section presents and discusses the results from how the EKF can correct for errors in states that do not have an actual measurement value to be differenced in the residual of the filter.

For each method described throughout Section 4.2 the initial simulations were conducted using an initial data set in which the filter's covariance matrix was tuned to produce the optimal performance. This tuning is discussed in the next section, which shows how the alterations of the various covariance matrices affect the filters performance. Table 5-1 represents the series of tests used to evaluate the performance of the filter for each respective method.

Table 5-1: Test Descriptions

\begin{tabular}{|c|c|}
\hline Test Number & Data Set Description \\
\hline Test 1 & Initial Data Set \\
\hline Test 2 & Validation Data Set 1 \\
\hline Test 3 & Validation Data Set 2 \\
\hline
\end{tabular}

The position and velocity data from both the INS and GPS have an error associated with it meaning that there is no "actual" position and velocity data for an error analysis, although the GPS data has a level of accuracy high enough for a comparison with the INS data. One can easily see with visual inspection of both the position and velocity the errors associated with both the GPS and INS data. The following plots depict the position errors coupled with the INS and GPS.

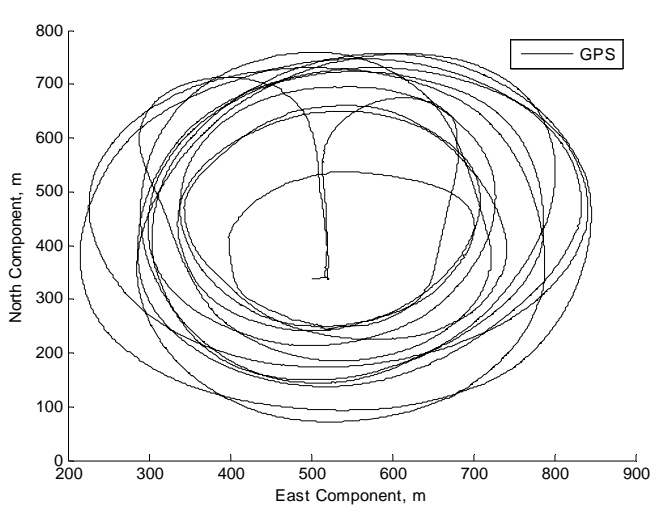

Figure 5.1: GPS Position

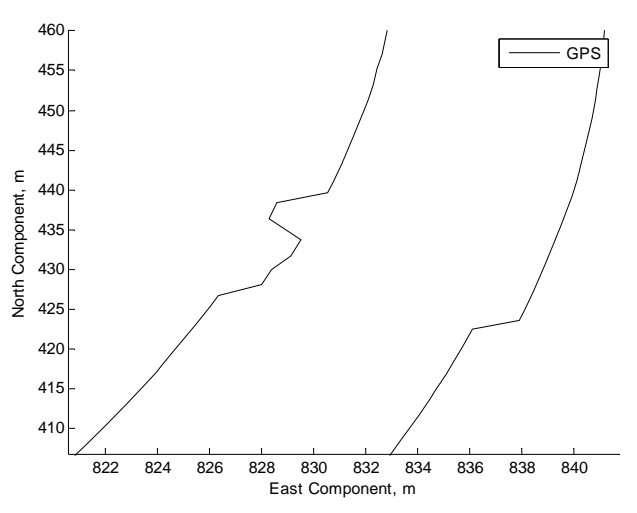

Figure 5.2: GPS Position (Magnified) 


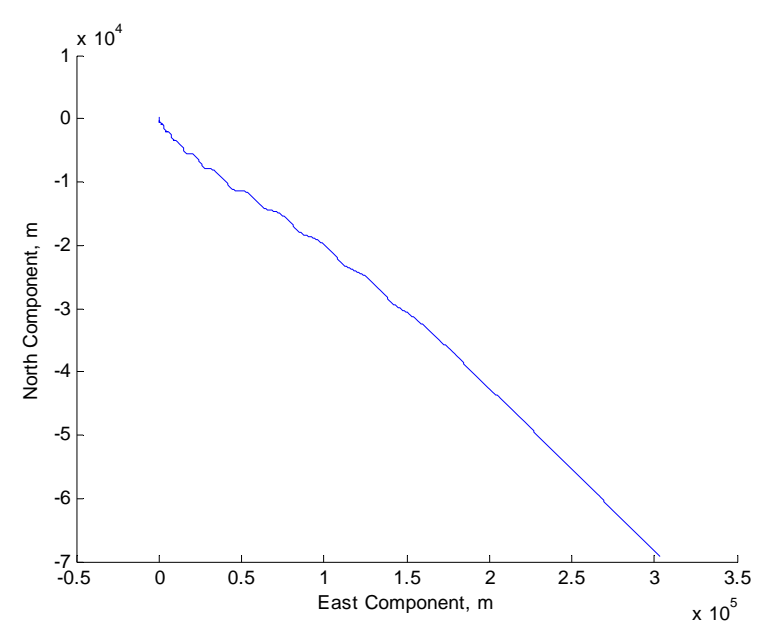

Figure 5.3: INS Position

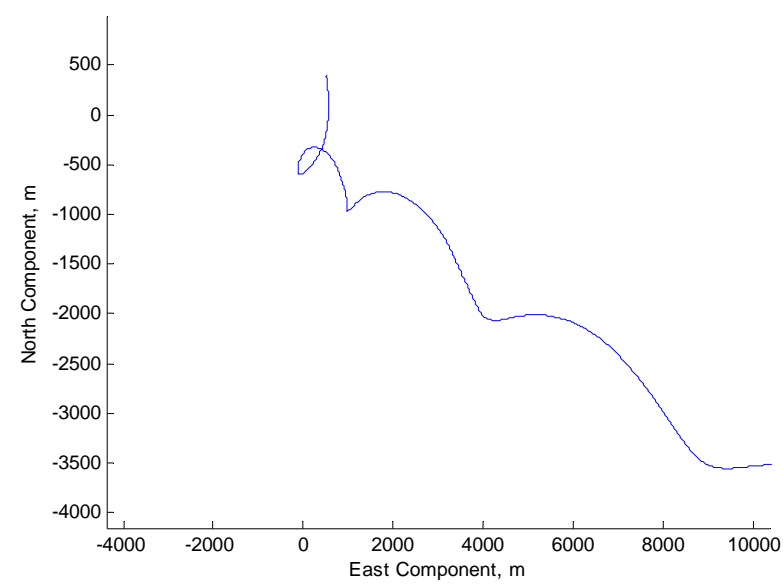

Figure 5.4: INS Position (Magnified)

These next plot contrasts the INS and GPS velocity against one another. The error in the INS velocity is quite evident while it's hard to distinguish between the errors in the GPS data.
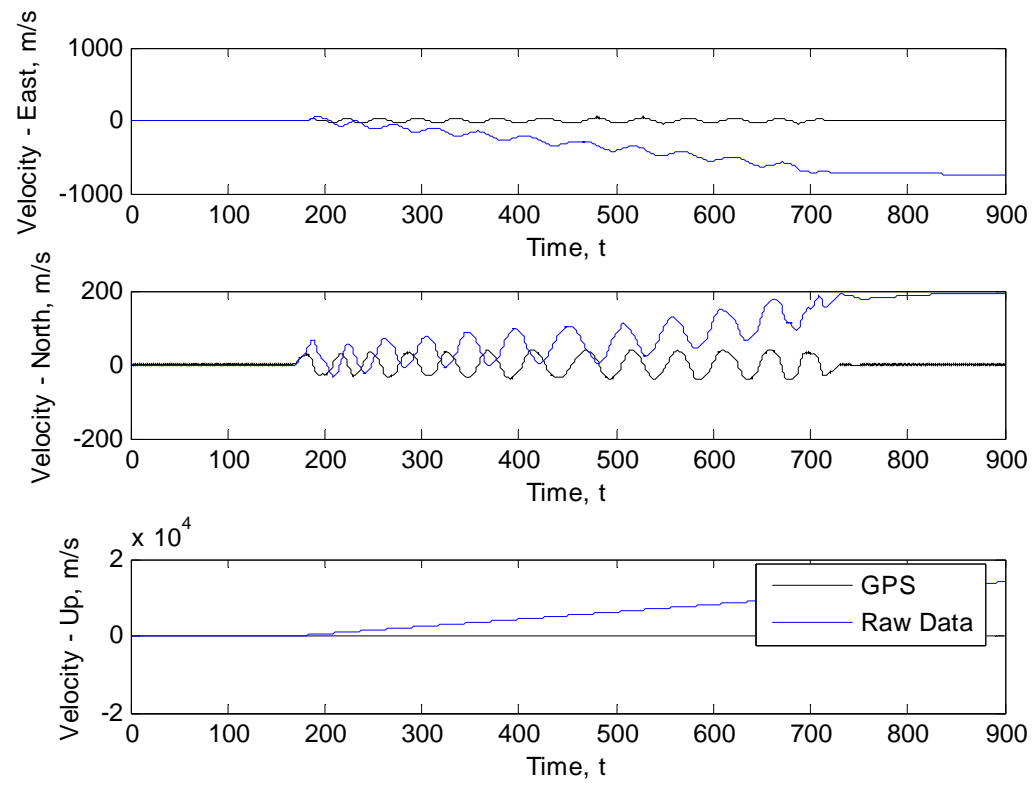

Figure 5.5: INS Data vs. GPS Velocity

The attitude values determined with the vertical gyro are at a level of precision high enough that they can be considered as the "actual" attitude values. With this being said, a more in-depth error analysis can be conducted on the filtered attitude data. The initial INS attitude values were shown in Chapter 3, although they are reiterated in Figure 
5.6 along with the error of the INS data over the dynamic response of the aircraft seen in

Figure 5.7
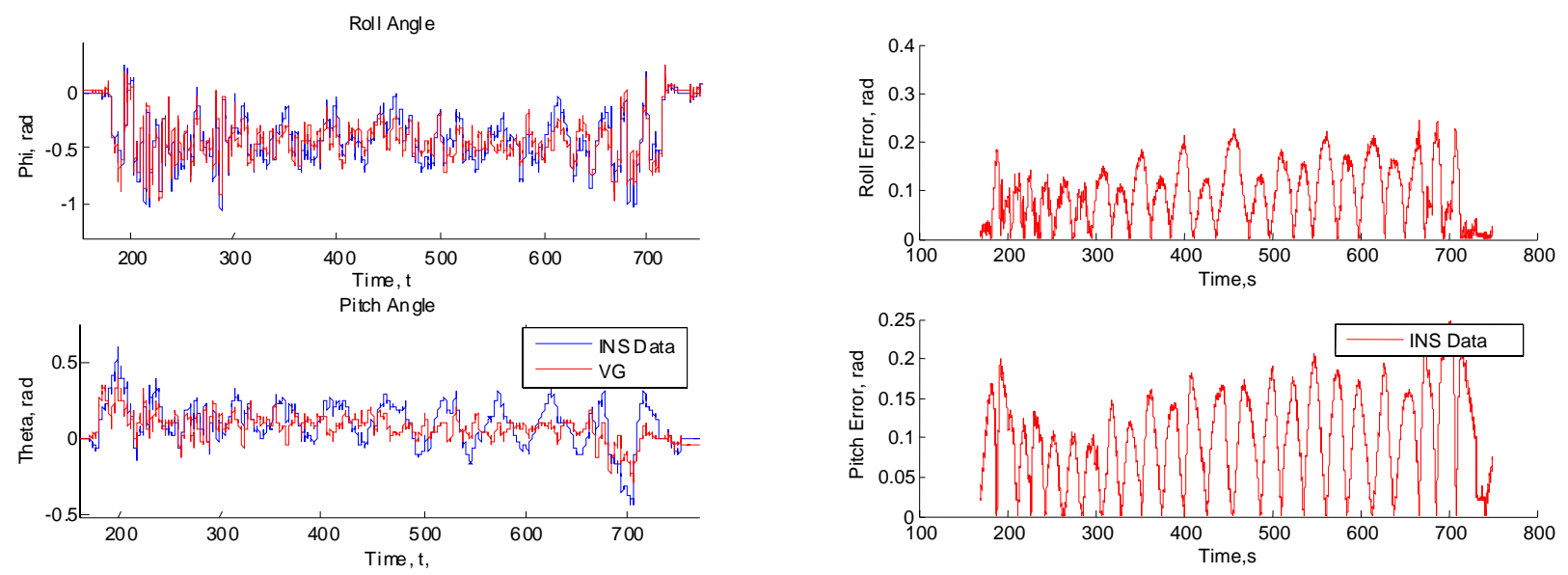

Figure 5.6: Vertical Gyro vs. INS Roll and Pitch Angle

Figure 5.7: Error Between Vertical Gyro Values and INS Data

The mean error and standard deviation from the vertical gyro values can be seen in Table 5-2 below, in which these values will be used later to determine how much correction has taken place throughout the filtering process.

Table 5-2: INS Error Analysis

\begin{tabular}{|c|c|c|c|c|c|c|}
\hline & \multicolumn{3}{|c|}{ Roll Angle } & \multicolumn{3}{c|}{ Pitch Angle } \\
\cline { 2 - 7 } & $\begin{array}{c}\text { Standard } \\
\text { Deviation (rad) }\end{array}$ & MSE & RMSE & $\begin{array}{c}\text { Standard } \\
\text { Deviation (rad) }\end{array}$ & MSE & RMSE \\
\hline Initial Data & 0.0502 & 0.0083 & 0.0911 & 0.0486 & 0.0087 & 0.0935 \\
\hline $\begin{array}{c}\text { Validation } \\
\text { Data Set 1 }\end{array}$ & 0.2019 & 0.1252 & 0.3539 & 0.2139 & 0.1414 & 0.3760 \\
\hline $\begin{array}{c}\text { Validation } \\
\text { Data Set 2 }\end{array}$ & 0.2851 & 0.1137 & 0.3372 & 0.2356 & 0.0712 & 0.2669 \\
\hline
\end{tabular}

\subsection{GPS/IMU/Vertical Gyro Sensor Fusion Simulation Results}

Within the context of this section, the actual benefit of the additional time and effort put forth to implement a Kalman filter in a navigation system algorithm can be clearly seen, even in the event where all of the necessary sensors are available to sustain autonomous control algorithms. If the system has the available computation power to add a filter, it is possible to further increase the precision of the data. Although the GPS provided sufficient position information for the formation fight program, the addition of the Kalman filter creates a considerable increase in data quality during times of GPS 
degradation. For the application simulated and compared in this thesis, the GPS and INS position and velocity of the aircraft from Section 4.2.3.1 are corrected, but this application could also be used in a wide range of applications, such as automobiles equipped with navigation systems, in which the GPS data obtained in the navigation system could be fused with that of the automobiles speed sensor allowing position and velocity information during times of GPS loss of signal (e.g. tunnels, thick foliage).

The main discussion for this method depicts how the alteration of the measurement and model/input covariance matrices directly influence the simulation results. As a starting point, the measurement covariance matrix, $R$, was set as the variance of the GPS position and velocity during a period when the vehicle was in a steady state period. Similarly the velocity positions in the diagonal of the model/input covariance matrix, $Q$, were set as the variance of the IMU's accelerometer during a steady state period. Again reiterating the point from Section 4.2.3.1 in which the position values in the diagonal were intentionally set to zero due to the fact that the position is a direct integration of the velocity. These initial values returned the following response, seen in Figure 5.8 and Figure 5.9, in regards to the velocity.
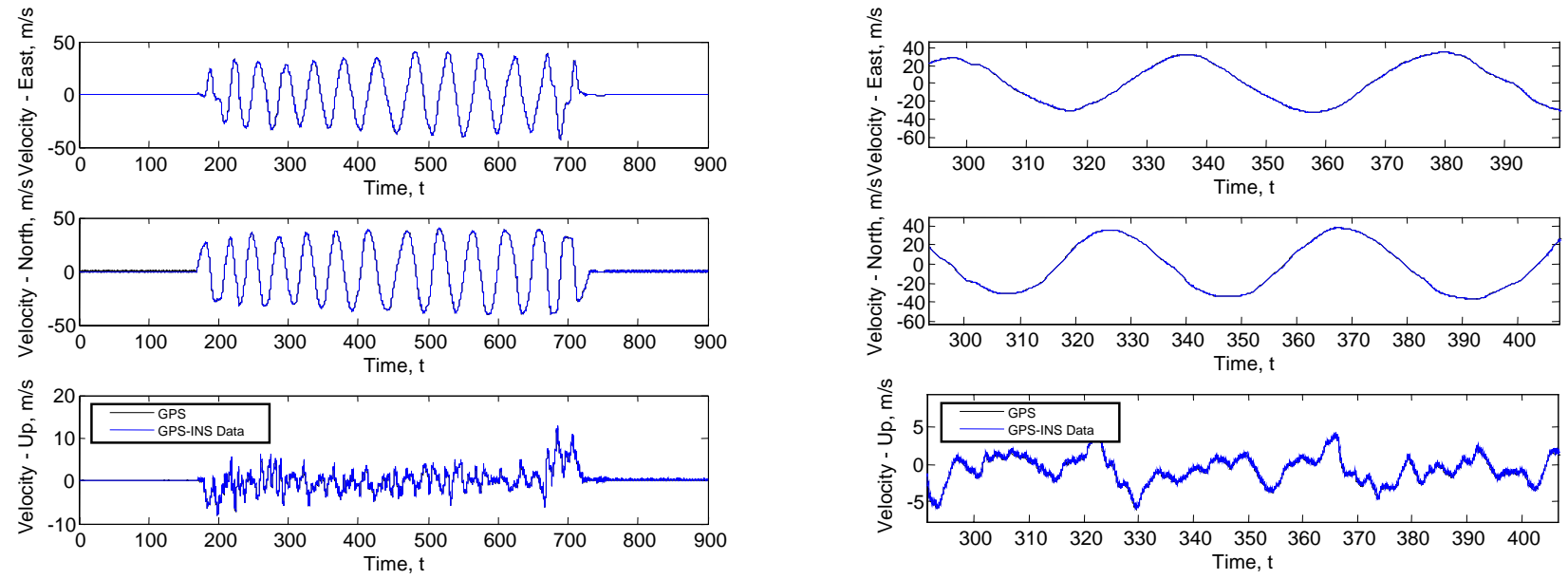

Figure 5.8: GPS vs. GPS-INS Velocity Plot (6 State)

Figure 5.9: GPS vs. GPS-INS Velocity Plot (Magnified - 6 State)

The simulation was also conducted with the accelerometer variance replacing the zero values in the position locations within the diagonal of the model/input covariance matrix, in which the results were degraded when compared to the simulation results of when the position values of the covariance matrix were set to zero. 
Although the velocity plots seem to perfectly mimic the GPS data, the actual correction can be seen when the filtered position data is plotted against the GPS data. Although there is no direct correction to the position from the model/input covariance matrix, the measurement covariance matrix reduces the error induced from the GPS values. These values are important for two reasons; firstly, without the variance values in the diagonal of the measurement covariance matrix, the noise from the GPS position wouldn't get estimated and removed from the GPS-INS filtered data, and more importantly, each state must have some sort of error modeling in one of the two covariance matrices or the error covariance matrix, $P$, will not be full rank driving the system to be singular. In other words, if there is no value in either of the model or measurement covariance matrices, that aspect of the system cannot be considered as a state in the filter. In Figure 5.10 and Figure 5.11 below it can now be clearly seen how well the filter has removed the drift from the INS while also removing the error from the GPS. Keep in mind that the INS's contribution to the position is the integration of the filtered velocity, while the GPS position error is modeled and removed from the system.

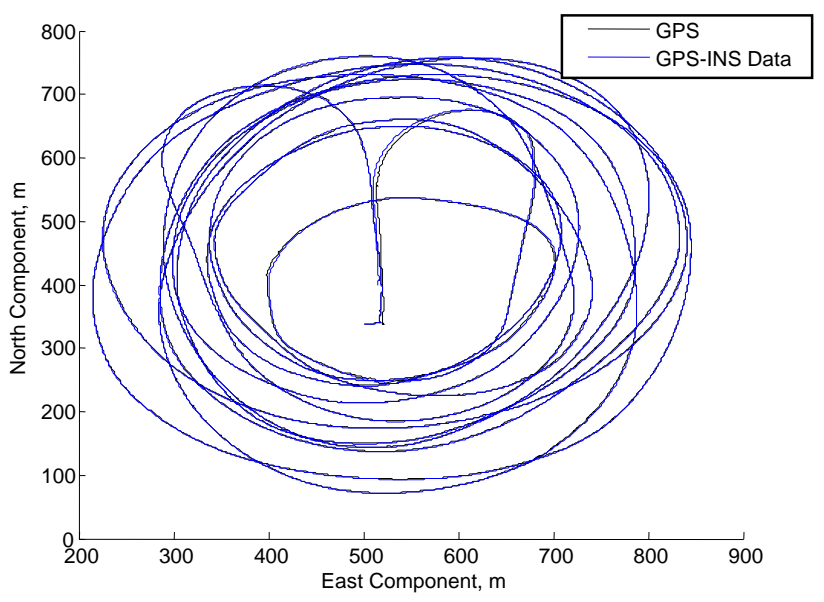

Figure 5.10: GPS vs. GPS-INS Data Position Plot (6 State)

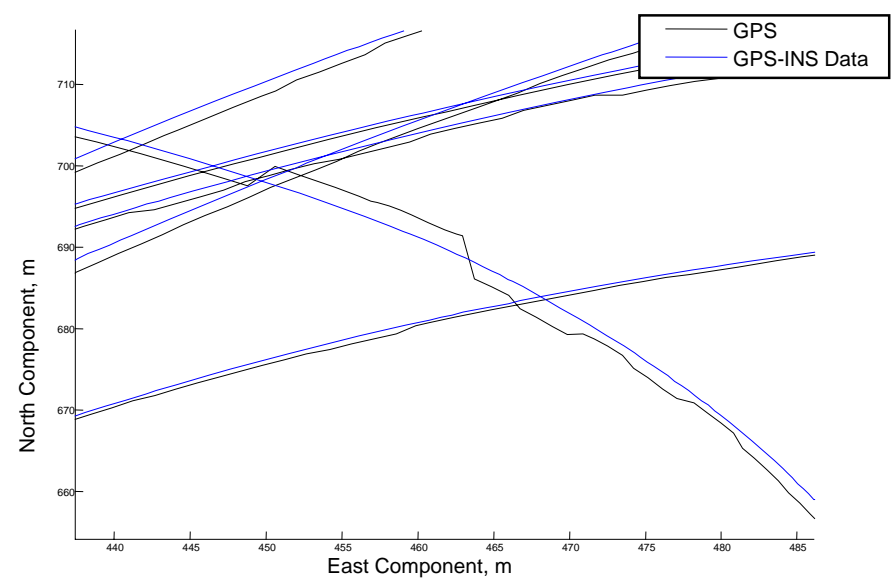

Figure 5.11: GPS vs. GPS-INS Data Position Plot (Magnified - 6 State)

If computation power is limited, the filter could also be reduced to a three state filter in which only the velocity is filtered, eliminating the position as a state within the filter. In this case the position would only be a direct integration of the velocity values and would still incorporate the GPS errors, which produces only a small contribution to the overall error in comparison to the INS drift error. The following plots represent the 
position of the three state filter in which a obvious increase in performance is still evident.

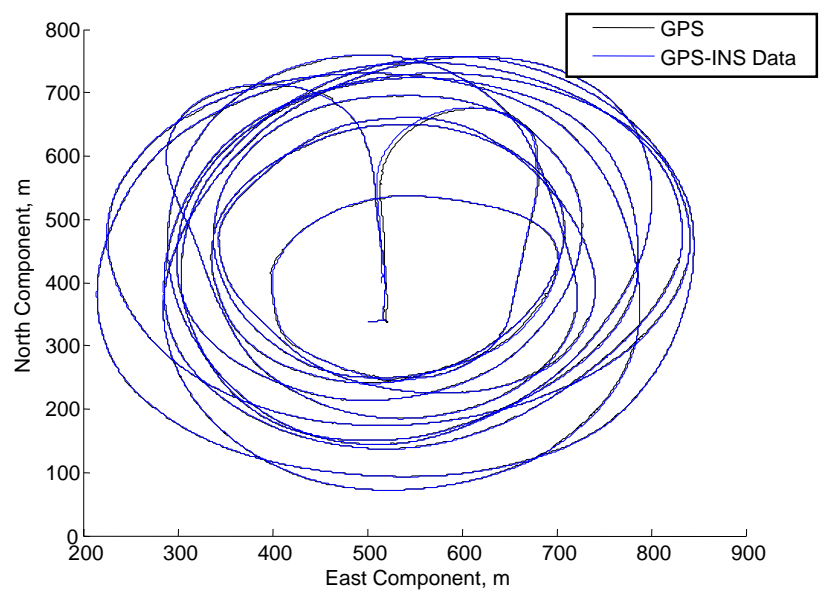

Figure 5.12: GPS vs. Filtered Data Position Plot (3 State)

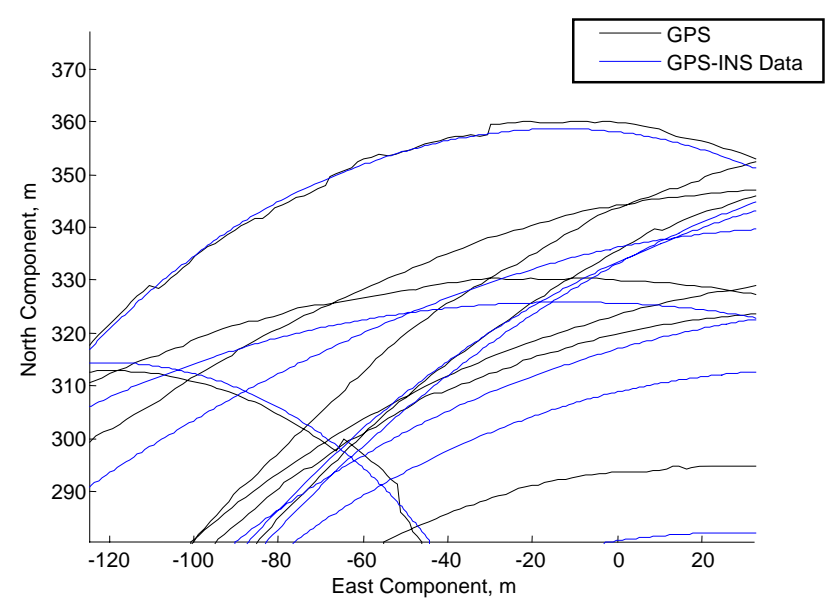

Figure 5.13: GPS vs. Filtered Data Position Plot (Magnified - 3 State)

As noted earlier the previous simulations utilized the model and measurement covariance values determined in Section 4.2.3.1, which was discussed, that varying these values could greatly affect the performance of the filter. The next few paragraphs will show the various affects and trends that occur from varying the model/input covariance matrix.

Again referencing back to Section 4.2.3.1, if the values in the model/system covariance were decreased so that the value was approaching zero, the system would put more weight on the INS estimated values, therefore more drifting error from the IMU data would be present in the output. This can be better visualized by viewing the Kalman gain calculation as a scalar, shown below:

$$
K=\frac{H P}{H^{2} P+R}
$$

To further elaborate on the scalar equation above, three possible scenarios are discussed to show how the dynamics of the filter respond when the main components are altered. The first case is when the error covariance matrix $(P)$ is considerably lower than that of the measurement covariance matrix $(R)$. This in turn causes the Kalman gain to increase, meaning that there is little error in the system, and the estimated states will be utilized. 
The second method discussed is when the measurement covariance matrix is considerably greater than that of the error covariance matrix, which means that the measurement value has a considerable amount of error (in relation to the error covariance matrix), which once again causes the system estimate to be weighted more heavily.

By decreasing the error covariance matrix by a factor of ten over four simulations, the dynamics of the first two cases can better be visualized. One can see how the system begins to incorporate the drift error back into the filter output in the following figures.

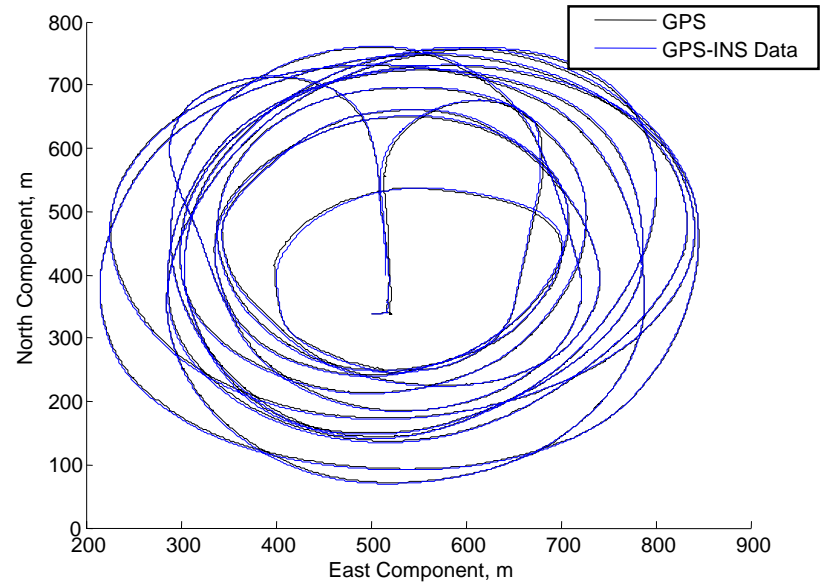

Figure 5.14: GPS vs. GPS-INS Data Position Plot (.1P)

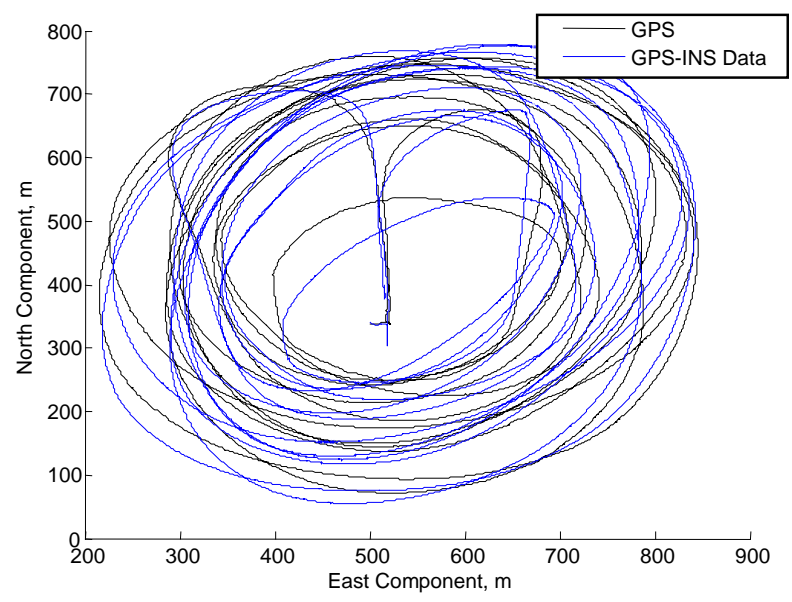

Figure 5.16: GPS vs. GPS-INS Data Position Plot (.001P)

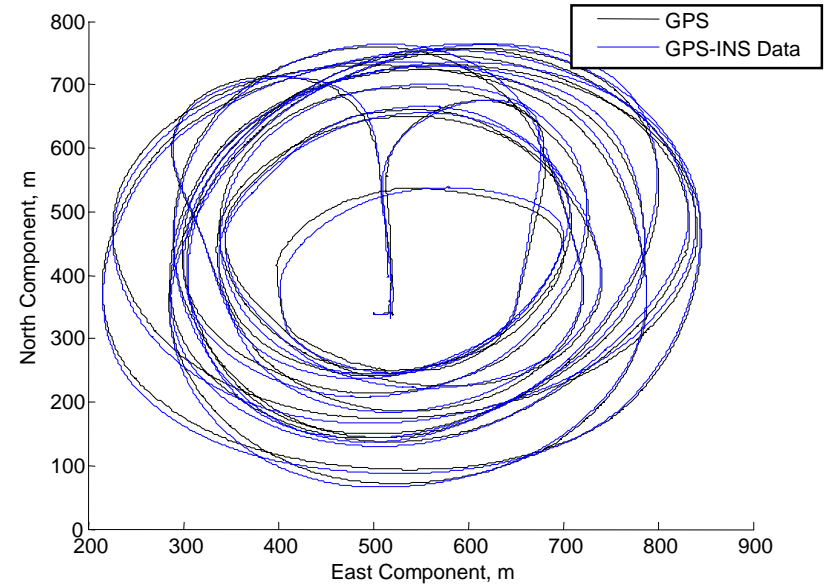

Figure 5.15: GPS vs. GPS-INS Data Position Plot (.01P)

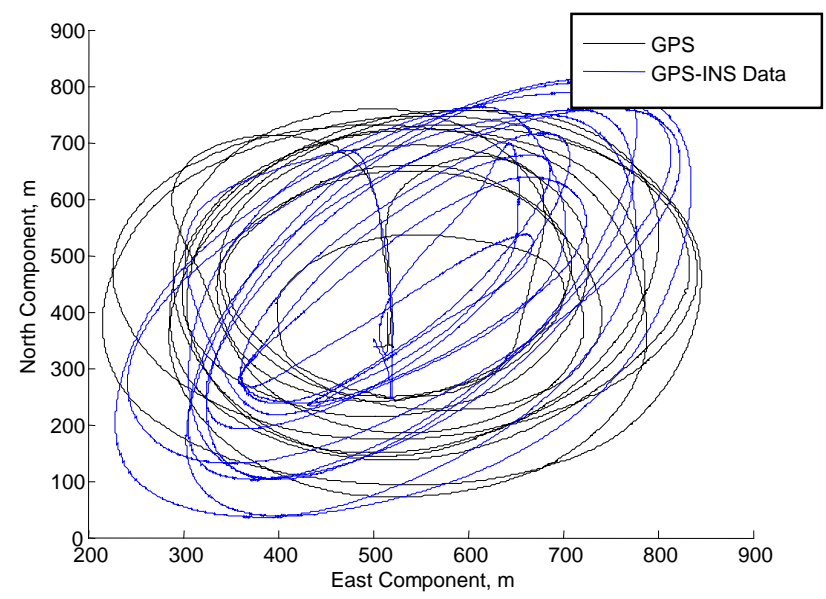

Figure 5.17: GPS vs. GPS-INS Data Position Plot (.0001P)

The final case discussed is when the covariance matrix is considerably greater than the measurement covariance matrix, in which the measurement covariance matrix 
can then be ignored (more weight on the measurement value) leaving only the GPS measurement value as seen in equation (5-2).

$$
K=\frac{H P}{H^{2} P}=\frac{1}{H} \Rightarrow \hat{x}=\hat{x}+\frac{1}{H}(z-H \hat{x})=\frac{z}{H}
$$

Therefore, as the covariance approaches infinity, the system would closer mimic the GPS values until the point where only GPS position and velocity is utilized and the INS is neglected. After the initial system has been established these values can be adjusted to suit the needs of the system. This tuning is explained and discussed further in the next chapter.

\subsection{GPS/GPS Attitude/IMU Sensor Fusion Simulation Results}

The ability of having all of the sensors needed to sustain autonomous flight is a luxury that may not always be possible within the scope of UAVs or MAVs. As to compensate for the lack of precise information, the available sensor data is combined to generate the level of precision needed. This section begins to show that the aircraft attitude can be corrected without the use of the vertical gyro through the use of the EKF. The simulation data discussed in the following paragraphs is the position, velocity, and attitude from the calculations described in Section 4.2.3.2 in which the GPS estimated attitude was used as a measurement value in the residual of the filter.

The following simulation is composed of nine states, in which a detailed error analysis will be conducted on the attitude corrections. Prior to the attitude discussion, the position and velocity is first shown in the following plots to show that there is no loss of quality to the data from the previous method. 

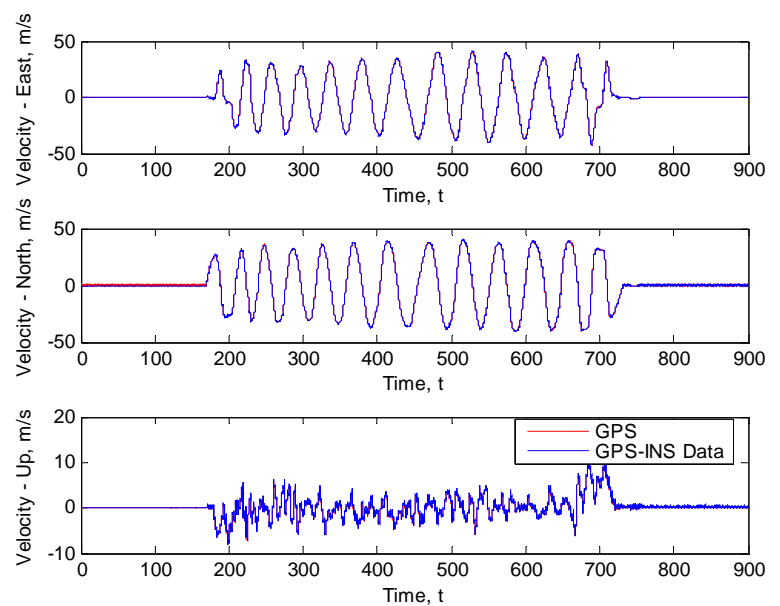

Figure 5.18: GPS vs. GPS-INS Data Velocity Plot (Method II)

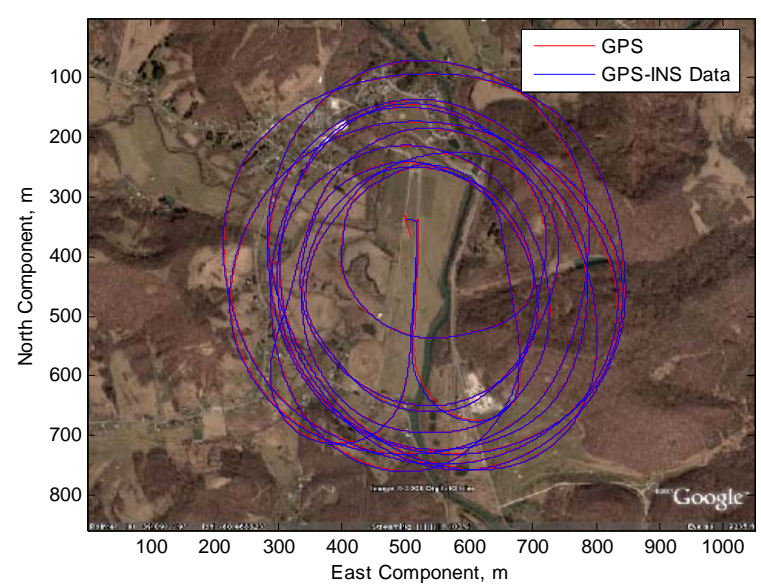

Figure 5.19: GPS vs. GPS-INS Data Position Plot (Method II)

The figures below compare the roll and pitch angles between the vertical gyro, filtered, and INS data along with magnified view so the one can see the actual reduction in error between the data sources. By visual inspection alone there is a clear decrease in error from the INS data and INS/GPS filtered data, in which the following paragraphs, tables, and figures further elaborate on the error numerically.

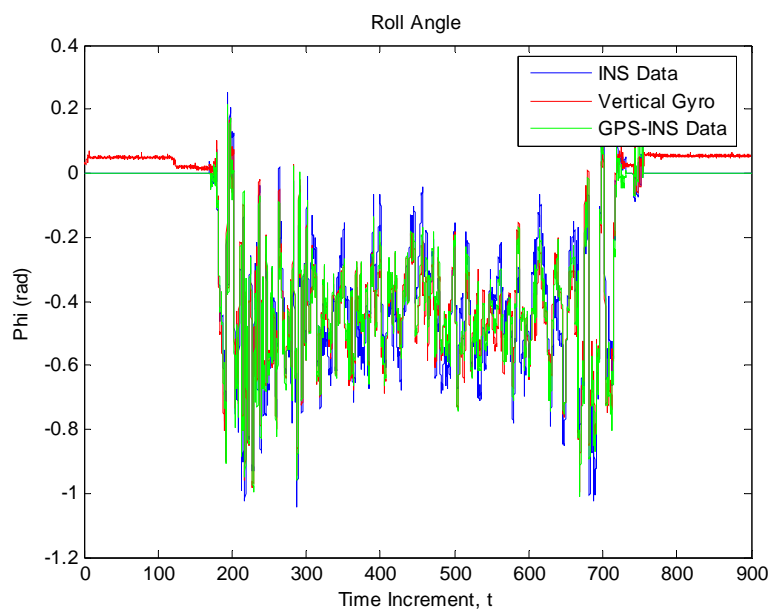

Figure 5.20: Vertical Gyro/INS Data/GPS-INS Filtered Data Roll Angle Comparison (Method II)

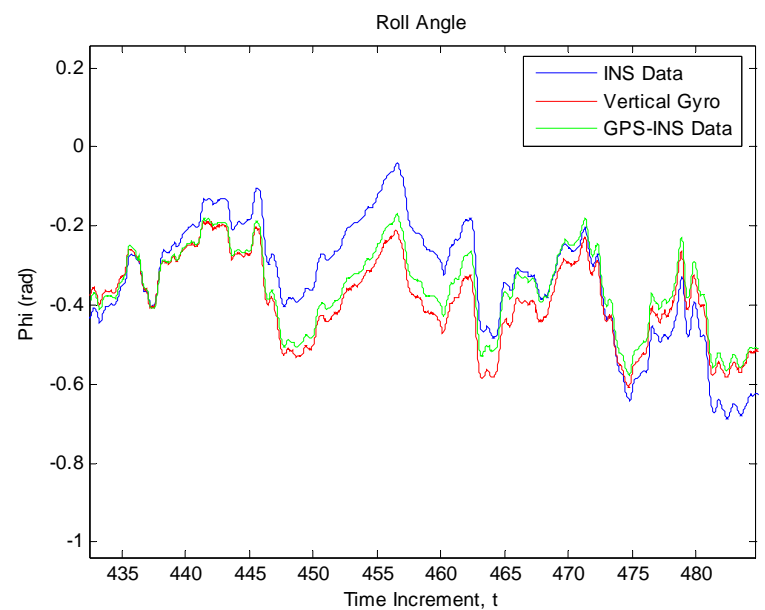

Figure 5.21: Vertical Gyro/INS Data/GPS-INS Data Roll Angle Comparison (Magnified Method II) 


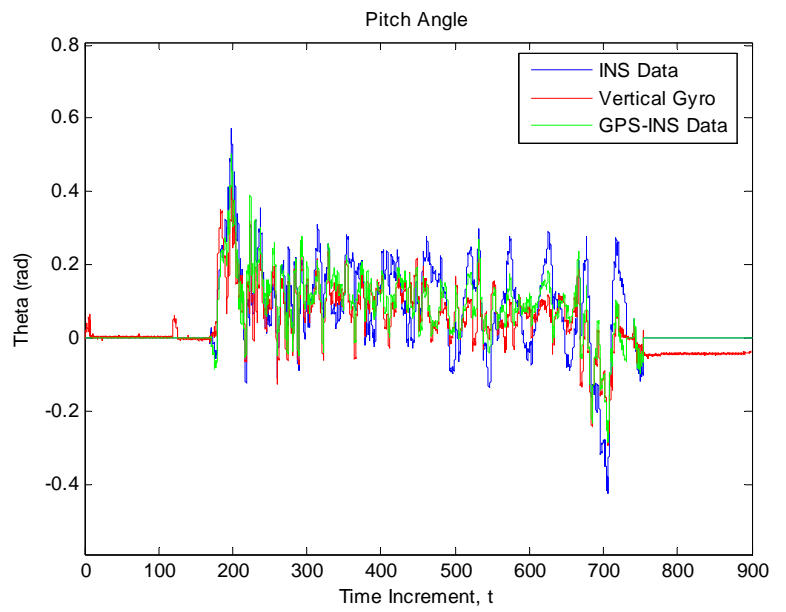

Figure 5.22: Vertical Gyro/INS Data/GPS-INS Filtered Data Roll Angle Comparison (Method II)

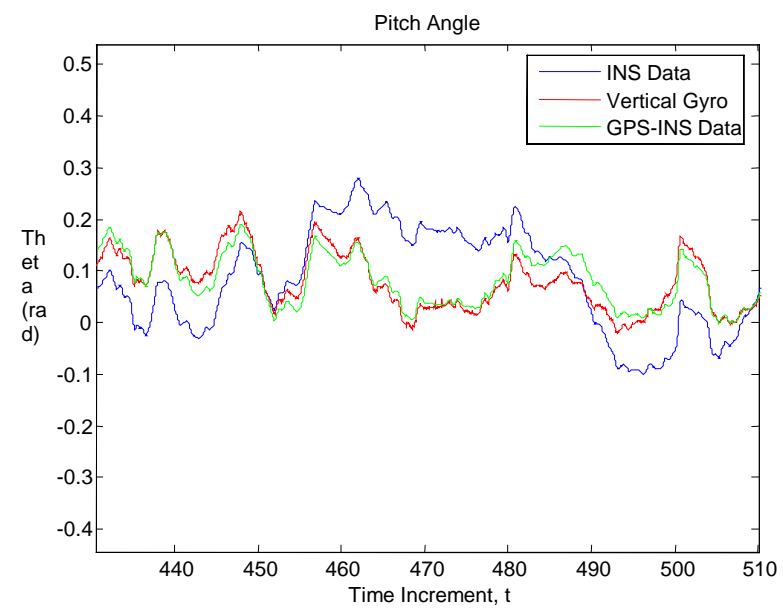

Figure 5.23: Vertical Gyro/INS Data/GPS-INS Filtered Data Roll Angle Comparison (Magnified - Method II)

Table 5-3 below compares the standard deviation (STD), mean squared error (MSE), and the root mean squared error (RMSE) between the GPS-INS filtered output and the INS roll and pitch values. As seen from the table, considerable improvement is achieved for each error calculation in which the roll standard deviation, MSE, and RMSE were each decreased by $0.0271,0.0069$, and 0.0536 from the raw INS integration. The pitch STD, MSE, and RMSE was also decreased by $0.0232,0.0073$, and 0.0561 from the raw INS integration.

Table 5-3: Method II Attitude Error Analysis (Initial Data Set)

\begin{tabular}{|c|c|c|c|c|c|c|c|}
\hline \multicolumn{2}{|c|}{} & \multicolumn{3}{c|}{ Roll Angle } & \multicolumn{3}{c|}{ Pitch Angle } \\
\cline { 3 - 8 } & STD (rad) & MSE & RMSE & STD (rad) & MSE & RMSE \\
\hline \multirow{2}{*}{$\begin{array}{c}\text { Initial } \\
\text { Data }\end{array}$} & $\begin{array}{c}\text { INS-GPS Data (9 } \\
\text { State) }\end{array}$ & 0.0231 & 0.0014 & 0.0375 & 0.0254 & 0.0014 & 0.0374 \\
\cline { 2 - 8 } & INS Data & 0.0502 & 0.0083 & 0.0911 & 0.0486 & 0.0087 & 0.0935 \\
\hline
\end{tabular}

To test the validity of the filter, two additional validation data test sets were simulated. The purpose of these additional data sets are to show that the filter hasn't been specifically tuned for this exact data set and that the it is capable of producing similar results under varying dynamic disturbances. The additional data sets simulated were obtained from the same aircraft and sensor configuration. Since the main focus of this research topic is to produce accurate aircraft attitude values equivalent to that of the vertical gyro, the position and velocity plots will be neglected during the evaluation of the validation data. 
Table 5-4 shows the error calculations after the first validation data set was simulated along with the initial data set for comparison purposes. As seen from the table, the second validation set also improved the raw INS data; in fact, the filter actually posted a higher level of error reduction from the initial data set. The roll STD, MSE, and RMSE were each decreased by $0.1562,0.1221$ and 0.2949 . The pitch STD, MSE, and RMSE was also decreased by $0.1581,0.1370$, and 0.3098 .

Table 5-4: Method II- Attiude Error Analysis (Validation Data Set 1)

\begin{tabular}{|c|c|c|c|c|c|c|c|}
\hline \multirow{2}{*}{} & \multicolumn{3}{|c|}{ Roll Angle } & \multicolumn{3}{c|}{ Pitch Angle } \\
\cline { 3 - 8 } & STD (rad) & MSE & RMSE & STD (rad) & MSE & RMSE \\
\hline \multirow{2}{*}{$\begin{array}{c}\text { Initial } \\
\text { Data }\end{array}$} & $\begin{array}{c}\text { INS-GPS Data } \\
\text { (9 State) }\end{array}$ & 0.0231 & 0.0014 & 0.0375 & 0.0254 & 0.0014 & 0.0374 \\
\cline { 2 - 8 } & INS Data & 0.0502 & 0.0083 & 0.0911 & 0.0486 & 0.0087 & 0.0935 \\
\hline \multirow{2}{*}{$\begin{array}{c}\text { Validation } \\
\text { Data Set 1 }\end{array}$} & $\begin{array}{c}\text { INS-GPS Data } \\
\text { (9 State) }\end{array}$ & 0.0457 & 0.0031 & 0.0590 & 0.0558 & 0.0044 & 0.0662 \\
\cline { 2 - 8 } & INS Data & 0.2019 & 0.1252 & 0.3539 & 0.2139 & 0.1414 & 0.3760 \\
\hline
\end{tabular}

Table 5-5 shows the error a calculation after the second validation data set was simulated along with the initial data and first validation sets for comparison purposes. As seen in the table, the second validation set produced results that improve the roll and pitch angles comparable to that of the first two sets simulated.

Table 5-5: Method II Attitude Error Analysis (Validation Data Set 2)

\begin{tabular}{|c|c|c|c|c|c|c|c|}
\hline \multirow{2}{*}{} & \multicolumn{3}{|c|}{ Roll Angle } & \multicolumn{3}{c|}{ Pitch Angle } \\
\cline { 3 - 8 } & STD (rad) & MSE & RMSE & STD (rad) & MSE & RMSE \\
\hline \multirow{2}{*}{$\begin{array}{c}\text { Initial } \\
\text { Data }\end{array}$} & $\begin{array}{c}\text { INS-GPS Data } \\
\text { (9 State) }\end{array}$ & 0.0231 & 0.0014 & 0.0375 & 0.0254 & 0.0014 & 0.0374 \\
\cline { 2 - 8 } & INS Data & 0.0502 & 0.0083 & 0.0911 & 0.0486 & 0.0087 & 0.0935 \\
\hline $\begin{array}{c}\text { Validation } \\
\text { Data Set 1 }\end{array}$ & $\begin{array}{c}\text { INS-GPS Data } \\
\text { (9 State) }\end{array}$ & 0.0457 & 0.0031 & 0.0590 & 0.0558 & 0.0044 & 0.0662 \\
\cline { 2 - 8 } & INS Data & 0.2019 & 0.1252 & 0.3539 & 0.2139 & 0.1414 & 0.3760 \\
\hline $\begin{array}{c}\text { Validation } \\
\text { Data Set 2 }\end{array}$ & $\begin{array}{c}\text { INS-GPS Data } \\
\text { (9 State) }\end{array}$ & 0.0718 & 0.0090 & 0.0946 & 0.0580 & 0.0078 & 0.0884 \\
\cline { 2 - 8 } & INS Data & 0.2851 & 0.1137 & 0.3372 & 0.2356 & 0.0712 & 0.2669 \\
\hline
\end{tabular}

The actual error between the GPS-INS filtered data/Vertical Gyro and the INS/Vertical Gyro was calculated at each dynamic measurement as depicted in Figure 5.24 . 

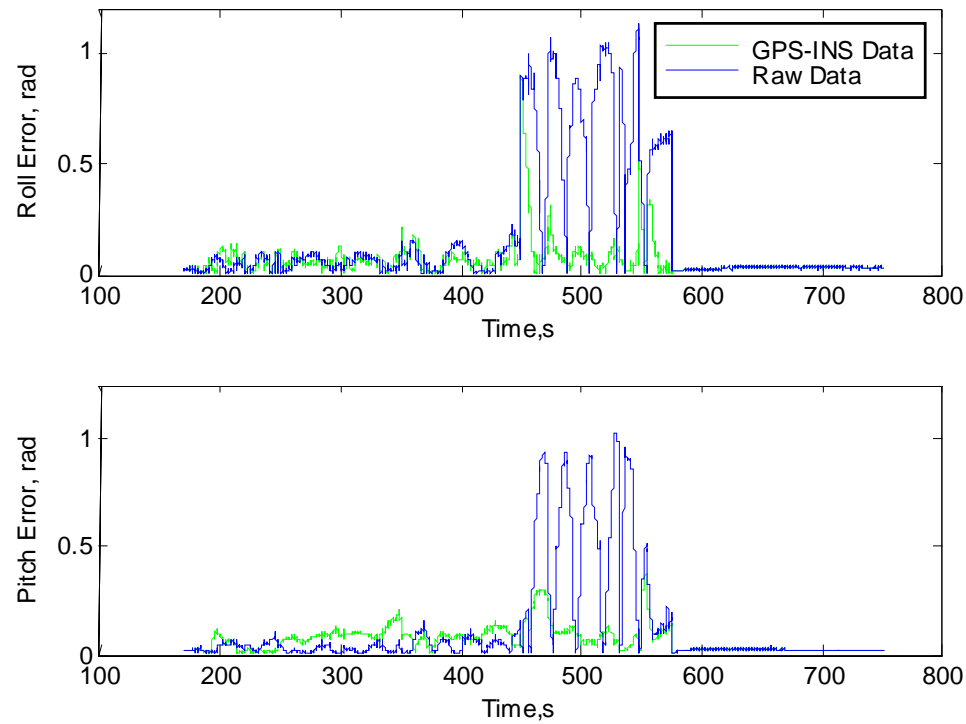

Figure 5.24: Validation Data Set 2 - Roll and Pitch Actual Error (Method II)

This analysis was conducted to show that even though the standard deviation MSE, and the RMSE decreased as a whole, the first 450 seconds of the pitch angle was actually better before the filtering process. The numbers are inflated due to the final 150 seconds of the simulation in which there is a large amount of error in the INS data. This is due to the fact that the filter induces additional error in the system during periods when there is little or no error in the system prior to filtering, for the filter always assumes there is some error due to the modeling of the system covariance matrix, $Q$. Although only three sets of data are simulated in this report, many data sets were tested in which this is the only case in which this occurrence was observed.

\subsection{1. $\quad$ Method II - Two-State Filter}

As stated earlier the previous simulation was composed of nine states, although similar to the previous section, the number of states in this system could also be reduced in the case that there is a need to conserve computation power. Given that each state has a measurement value associated with it within the residual, meaning the observation matrix, $H$, is of full rank, the number of states could be reduced to as few as two. In which the two remaining states within the filter are roll and pitch angle, while the heading angle is determined with the use of the 'east' and 'north' GPS velocity vector. 
The following figures compare the three data sources in which the two-state filter was implemented to filter the roll and pitch angle. Upon visual inspection of the plots, one can see that the two-state filter produces results comparable to that of the nine-state filter and could be very effective in small UAVs or MAVs.

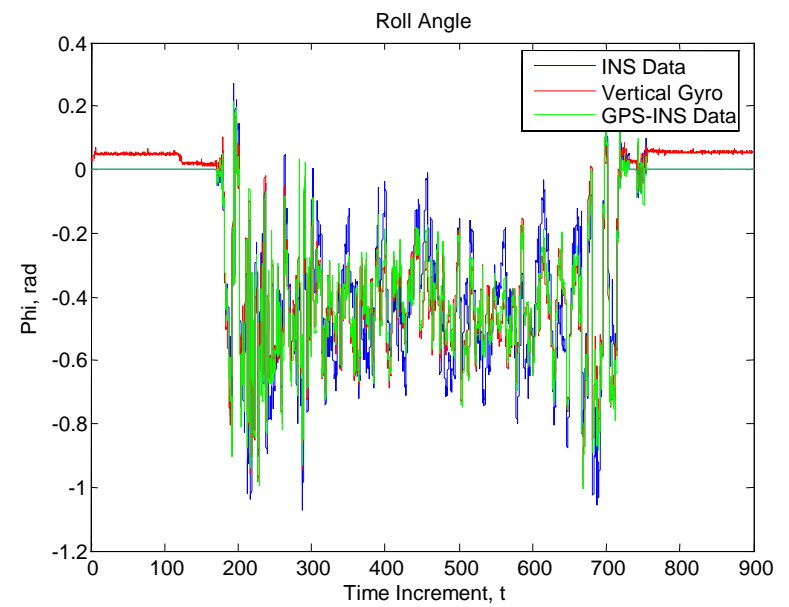

Figure 5.25: Vertical Gyro/INS Data/GPS-INS Filtered Data Roll Angle Comparison (Method II - 2 State Fitler)

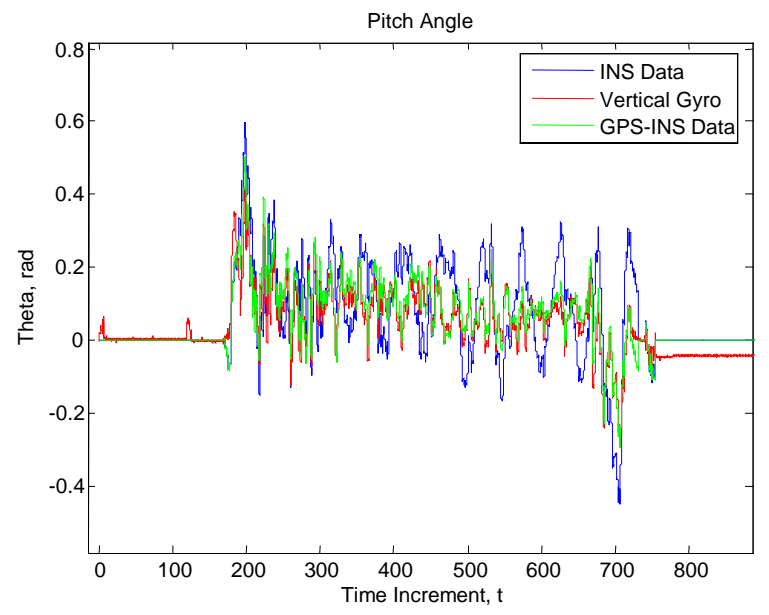

Figure 5.27: Vertical Gyro/INS Data/GPS-INS Filtered Data Pitch Angle Comparison (Method II - 2 State Fitler)

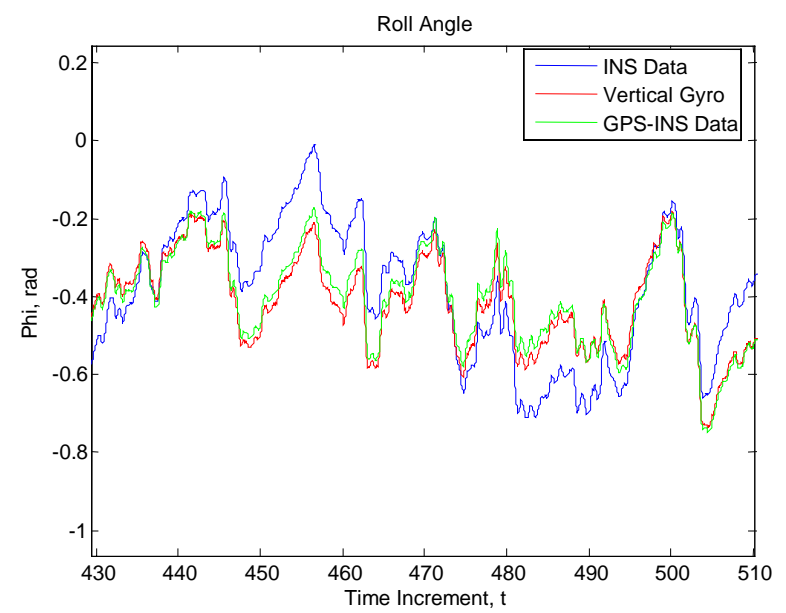

Figure 5.26: Vertical Gyro/INS Data/GPS-INS Filtered Data Roll Angle Comparison (Magnified - Method II - 2 State Filter)

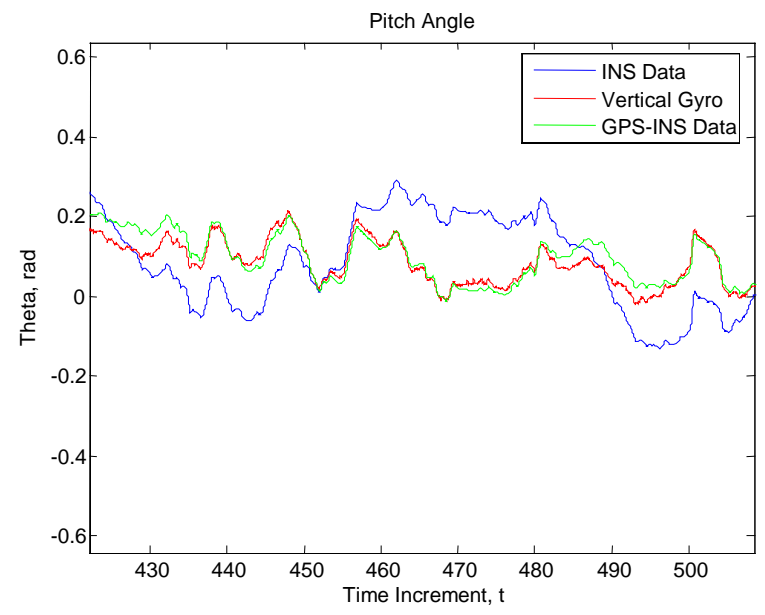

Figure 5.28: Vertical Gyro/INS Data/GPS-INS Filtered Data Pitch Angle Comparison (Magnified - Method II - 2 State Filter)

Table 5-6 shows the error calculations from the two-state system, such that all three simulated data sets where contrasted against one another. Similar to the nine-state filter, the two-state filter also posted a significant decrease in error which proves to be useful in the situation where there is limited computational ability. 
Table 5-6: Method II Attitude Error Analysis (2 State)

\begin{tabular}{|c|c|c|c|c|c|c|c|}
\hline & & \multicolumn{3}{|c|}{ Roll Angle } & \multicolumn{3}{|c|}{$\begin{array}{l}\text { Pitch Angle } \\
\end{array}$} \\
\hline & & STD (rad) & MSE & RMSE & STD (rad) & MSE & RMSE \\
\hline \multirow[t]{2}{*}{ Initial Data } & $\begin{array}{c}\text { INS-GPS } \\
\text { Data } \\
\text { (2 State) }\end{array}$ & 0.0198 & 0.0015 & 0.0387 & 0.0248 & 0.0016 & 0.0404 \\
\hline & INS Data & 0.0502 & 0.0083 & 0.0911 & 0.0486 & 0.0087 & 0.0935 \\
\hline \multirow[t]{2}{*}{$\begin{array}{l}\text { Validation Data } \\
\text { Set } 1\end{array}$} & $\begin{array}{c}\text { INS-GPS } \\
\text { Data } \\
\text { (2 State) }\end{array}$ & 0.0452 & 0.0064 & 0.0801 & 0.0547 & 0.0091 & 0.0953 \\
\hline & INS Data & 0.2019 & 0.1252 & 0.3539 & 0.2139 & 0.1414 & 0.3760 \\
\hline \multirow[t]{2}{*}{$\begin{array}{l}\text { Validation Data } \\
\text { Set } 2\end{array}$} & $\begin{array}{c}\text { INS-GPS } \\
\text { Data } \\
\text { (2 State) }\end{array}$ & 0.0720 & 0.0211 & 0.1454 & 0.0608 & 0.0105 & 0.1026 \\
\hline & INS Data & 0.2851 & 0.1137 & 0.3372 & 0.2356 & 0.0712 & 0.2669 \\
\hline
\end{tabular}

Due to the repetitive nature of the validation data sets, the remaining results and error analysis can be found in Appendix A, although Table 5-7 be was constructed to compare the full-state INS with the two-state INS.

Table 5-7: Method II Data Comparison

\begin{tabular}{|c|c|c|c|c|c|c|c|}
\hline & \multicolumn{3}{|c|}{ Roll Angle } & \multicolumn{3}{|c|}{ Pitch Angle } \\
\hline & & STD (rad) & MSE & RMSE & STD (rad) & MSE & RMSE \\
\hline \multirow{3}{*}{ Initial Data } & $\begin{array}{c}\text { INS-GPS Data } \\
\text { (9 State) }\end{array}$ & 0.0231 & 0.0014 & 0.0375 & 0.0254 & 0.0014 & 0.0374 \\
\hline & $\begin{array}{c}\text { INS-GPS Data } \\
\text { (2 State) }\end{array}$ & 0.0198 & 0.0015 & 0.0387 & 0.0248 & 0.0016 & 0.0404 \\
\hline & INS Data & 0.0502 & 0.0083 & 0.0911 & 0.0486 & 0.0087 & 0.0935 \\
\hline \multirow{3}{*}{$\begin{array}{l}\text { Validation } \\
\text { Data Set } 1\end{array}$} & $\begin{array}{c}\text { INS-GPS Data } \\
\text { (9 State) }\end{array}$ & 0.0457 & 0.0031 & 0.0590 & 0.0558 & 0.0044 & 0.0662 \\
\hline & $\begin{array}{c}\text { INS-GPS Data } \\
\text { (2 State) }\end{array}$ & 0.0452 & 0.0064 & 0.0801 & 0.0547 & 0.0091 & 0.0953 \\
\hline & INS Data & 0.2019 & 0.1252 & 0.3539 & 0.2139 & 0.1414 & 0.3760 \\
\hline \multirow{3}{*}{$\begin{array}{l}\text { Validation } \\
\text { Data Set } 2\end{array}$} & $\begin{array}{c}\text { INS-GPS Data } \\
\text { (9 State) }\end{array}$ & 0.0718 & 0.0090 & 0.0946 & 0.0580 & 0.0078 & 0.0884 \\
\hline & $\begin{array}{c}\text { INS-GPS Data } \\
(2 \text { State })\end{array}$ & 0.0720 & 0.0211 & 0.1454 & 0.0608 & 0.0105 & 0.1026 \\
\hline & INS Data & 0.2851 & 0.1137 & 0.3372 & 0.2356 & 0.0712 & 0.2669 \\
\hline
\end{tabular}

As seen from the table, the initial data set showed nearly the same reduction in error between the two and nine-state filters. By then contrasting the MSE and RMSE of the two validation sets, it can be inferred that the nine-state filter generates slightly better results, although the STD reduction is consistently the same over all of the data sets. By removing the additional states for the two-state filter, the position, velocity, and heading 
correction is removed, causing a slight reduction in the two-state filter's performance, which is evident from the previous table.

It can also be stated that the filter proved to remain effective with only one of the GPS estimated attitude values used within the filtering algorithm. Since the roll and pitch are coupled in the state transition matrix, as discussed in Section 4.2.3.3, only one value is needed in the measurement covariance matrix so that the noise can be reduced in the system.

\subsection{GPS/IMU Sensor Fusion Simulation Results}

The third method utilizes the GPS and IMU data to improve the attitude values without the use of the GPS attitude estimates within the residual. As discussed in Section 4.2.3.3, without the GPS attitude there is no direct compensation for the attitude, causing the correction to be achieved from the coupling effects in the DCM, which transforms the INS position and velocity from the body axis to the ECEF axis. Unlike the previous method, the system cannot be reduced to any less than six states since the attitude correction is being carried out via the velocity data.

The following table depicts the error analysis for the initial and two validation data sets, such that it is again evident from the table that this method improves the attitude results.

Table 5-8: Method III Attitude Error Analysis

\begin{tabular}{|c|c|c|c|c|c|c|c|}
\hline \multirow{2}{*}{} & \multicolumn{3}{|c|}{ Roll Angle } & \multicolumn{3}{c|}{ Pitch Angle } \\
\cline { 3 - 8 } & & STD (rad) & MSE & RMSE & STD (rad) & MSE & RMSE \\
\hline \multirow{2}{*}{ Initial Data } & Method III & 0.0350 & 0.0015 & 0.0387 & 0.0286 & 0.0016 & 0.0404 \\
\cline { 2 - 8 } & INS Data & 0.0502 & 0.0083 & 0.0911 & 0.0486 & 0.0087 & 0.0935 \\
\hline \multirow{2}{*}{ Validation Data Set 1 } & Method III & 0.0688 & 0.0064 & 0.0801 & 0.0836 & 0.0091 & 0.0953 \\
\cline { 2 - 8 } & INS Data & 0.2019 & 0.1252 & 0.3539 & 0.2139 & 0.1414 & 0.3760 \\
\hline \multirow{2}{*}{ Validation Data Set 2 } & Method III & 0.1178 & 0.0211 & 0.3372 & 0.0859 & 0.0105 & 0.1026 \\
\cline { 2 - 8 } & INS Data & 0.2851 & 0.1137 & 0.3372 & 0.2356 & 0.0712 & 0.2669 \\
\hline
\end{tabular}

Figure 5.29 shows the filtered attitude comparison between the INS/GPS-INS filtered data in which it is clear from visual inspection that the data is similar to that of the vertical gyro. Although, keep in mind that this method's attitude correction is totally 
reliant on the fact that the linearized DCM is coupled with the position and velocity. Which means that the error associated with the higher order term's from the Taylor series expansion during the linearization process are not compensated for, this can possibly cause the this method to not post as high of error reduction as the previous method. The other issue that can decrease the efficiency of the filter is the determination of the covariance matrix. These will be revisited later when the results of all the methods are contrasted against one another.
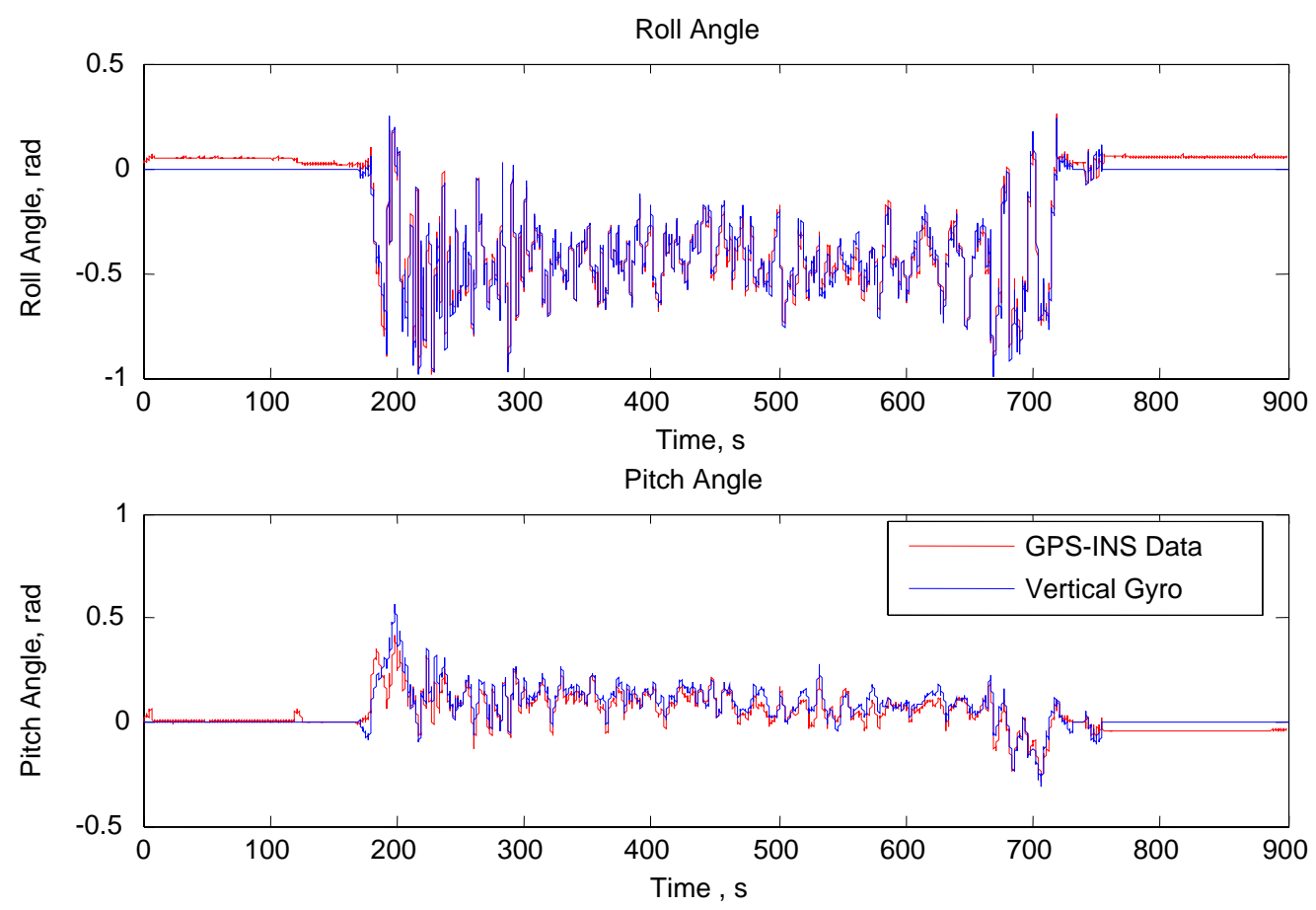

Figure 5.29: Vertical Gyro/GPS-INS Comparison

Due to the repetitive nature of the validation data sets, the remaining results and error analysis can be found in Appendix B.

\subsection{Method IV - Combination of Method II and III}

This final method combines methods II and III to allow the GPS attitude and DCM Jacobian matrix to correct the position, velocity, and attitude as discussed in Section 4.2.3.4. As with the previous method, the state transition matrix must remain at least a $6 x 6$ matrix, which is comprised of attitude and velocity values at a minimum to allow for the Kalman filter to compensate for the attitudes. Again, this is due to fact that 
the correction was carried out in regards to the DCM and also relies on the GPS estimated attitude components.

Table 5-9 shows the error calculations conducted on each of the three data sets in which it can be seen that a reduction in error is evident.

Table 5-9: Method IV Attitude Error Analysis

\begin{tabular}{|l|c|c|c|c|c|c|c|}
\hline \multirow{2}{*}{} & \multicolumn{3}{|c|}{ Roll Angle } & \multicolumn{3}{c|}{ Pitch Angle } \\
\cline { 3 - 8 } & $\begin{array}{c}\text { Standard } \\
\text { Deviation } \\
\text { (rad) }\end{array}$ & MSE & RMSE & $\begin{array}{c}\text { Standard } \\
\text { Deviation } \\
\text { (rad) }\end{array}$ & MSE & RMSE \\
\hline \multirow{2}{*}{ Initial Data } & $\begin{array}{c}\text { Method } \\
\text { IV }\end{array}$ & 0.0233 & 0.0014 & 0.0374 & 0.0270 & 0.0015 & 0.0386 \\
\cline { 2 - 8 } & INS Data & 0.0502 & 0.0083 & 0.0911 & 0.0486 & 0.0087 & 0.0935 \\
\hline \multirow{2}{*}{$\begin{array}{l}\text { Validation } \\
\text { Data Set 1 }\end{array}$} & $\begin{array}{c}\text { Method } \\
\text { IV }\end{array}$ & 0.0535 & 0.0040 & 0.0635 & 0.0655 & 0.0056 & 0.0748 \\
\cline { 2 - 8 } & INS Data & 0.2019 & 0.1252 & 0.3539 & 0.2139 & 0.1414 & 0.3760 \\
\hline \multirow{2}{*}{$\begin{array}{l}\text { Validation } \\
\text { Data Set 2 }\end{array}$} & $\begin{array}{c}\text { Method } \\
\text { IV }\end{array}$ & 0.0915 & 0.0132 & 0.1148 & 0.0655 & 0.0072 & 0.0848 \\
\cline { 2 - 8 } & INS Data & 0.2851 & 0.1137 & 0.3372 & 0.2356 & 0.0712 & 0.2669 \\
\hline
\end{tabular}

Similar to the previous section, this combination of the methods also incorporates the error associated with the higher order terms from the DCM and kinematic equations linearization, although the GPS attitude estimates are also utilized as actual measurement values. The fact that the GPS attitude is being utilized in the residual could help reduce the error associated with the neglected linearization error.

The remaining data set simulations are located in Appendix $\mathrm{C}$, due to the redundant nature of the simulations.

\subsection{Method Comparisons and Discussions}

With the individual results shown and discussed in the previous sections, a comparison was conducted to determine which individual method produced the most effective results. Table 5-10 contrasts the roll and pitch attitude between method II (nine and two-state), method III, and finally the combination of methods II and III (method IV) for the initial, validation set 1 , and validation set 2 . Method I was neglected because its purpose is to correct position and velocity errors only. 
Table 5-10: Inter-Method Error Comparison

\begin{tabular}{|c|c|c|c|c|c|c|c|}
\hline & & \multicolumn{3}{|c|}{ Roll Angle } & \multicolumn{3}{|c|}{ Pitch Angle } \\
\hline & & $\begin{array}{c}\text { Standard } \\
\text { Deviation } \\
\text { (rad) }\end{array}$ & MSE & RMSE & $\begin{array}{c}\text { Standard } \\
\text { Deviation (rad) }\end{array}$ & MSE & RMSE \\
\hline \multirow{5}{*}{$\begin{array}{c}\text { Initial } \\
\text { Data }\end{array}$} & $\begin{array}{c}\text { INS-GPS } \\
\text { Data (9 State) }\end{array}$ & 0.0231 & 0.0014 & 0.0375 & 0.0254 & 0.0014 & 0.0374 \\
\hline & $\begin{array}{c}\text { INS-GPS } \\
\text { Data (2 State) }\end{array}$ & 0.0198 & 0.0015 & 0.0387 & 0.0248 & 0.0016 & 0.0404 \\
\hline & Method III & 0.0350 & 0.0015 & 0.0387 & 0.0286 & 0.0016 & 0.0404 \\
\hline & Method IV & 0.0233 & 0.0014 & 0.0374 & 0.0270 & 0.0015 & 0.0386 \\
\hline & INS Data & 0.0502 & 0.0083 & 0.0911 & 0.0486 & 0.0087 & 0.0935 \\
\hline \multirow{5}{*}{$\begin{array}{l}\text { Validation } \\
\text { Data Set } 1\end{array}$} & $\begin{array}{c}\text { INS-GPS } \\
\text { Data (9 State) }\end{array}$ & 0.0457 & 0.0031 & 0.0590 & 0.0558 & 0.0044 & 0.0662 \\
\hline & $\begin{array}{c}\text { INS-GPS } \\
\text { Data (2 State) }\end{array}$ & 0.0452 & 0.0064 & 0.0801 & 0.0547 & 0.0091 & 0.0953 \\
\hline & Method III & 0.0688 & 0.0064 & 0.0801 & 0.0836 & 0.0091 & 0.0953 \\
\hline & Method IV & 0.0535 & 0.0040 & 0.0635 & 0.0655 & 0.0056 & 0.0748 \\
\hline & INS Data & 0.2019 & 0.1252 & 0.3539 & 0.2139 & 0.1414 & 0.3760 \\
\hline \multirow{5}{*}{$\begin{array}{l}\text { Validation } \\
\text { Data Set } 2\end{array}$} & $\begin{array}{c}\text { INS-GPS } \\
\text { Data (9 State) }\end{array}$ & 0.0718 & 0.0090 & 0.0946 & 0.0580 & 0.0078 & 0.0884 \\
\hline & $\begin{array}{c}\text { INS-GPS } \\
\text { Data (2 State) }\end{array}$ & 0.0720 & 0.0211 & 0.1454 & 0.0608 & 0.0105 & 0.1026 \\
\hline & Method III & 0.1178 & 0.0211 & 0.3372 & 0.0859 & 0.0105 & 0.1026 \\
\hline & Method IV & 0.0915 & 0.0132 & 0.1148 & 0.0655 & 0.0072 & 0.0848 \\
\hline & INS Data & 0.2851 & 0.1137 & 0.3372 & 0.2356 & 0.0712 & 0.2669 \\
\hline
\end{tabular}

As seen from the previous table, areas highlighted with red depict when a single method clearly produces the highest level of reduction in error, while areas highlighted with orange depict when multiple methods produce the highest level of error reduction in which no single method can be determined to produce the best overall results. By evaluating the initial data set in Table 5-10 no single method can clearly be labeled as producing the best results as each method's amount of error reduction is similar, this could be due to the fact that each filter was tuned to its optimal performance for the initial set. By comparing the additional validation data sets, the filter posting the best results could be determined.

From the comparison from the two and nine-state filters from Section 5.3 it is already known that the nine-state filter produces a higher level of error reduction when simulated over various data sets. The question now is whether the deductions made for methods III and IV, in regards to the error associated with the higher order terms, hold true. By reviewing Table 5-10 again and comparing the results from each method, it can be seen that there is a definite decrease in performance from the best results from method 
II (nine state) and that of the third and fourth method in which method III produced the lowest level of error reduction which can most likely be attributed to the error associated with the linearization of the nonlinear terms, while method IV is also effected by the linearization error, some of this error is removed in the filtering process due to the GPS attitude.

In an additional note, the filtering process tends to visually produce better results for the roll angle than that of the pitch angle (Figure 5.30), although when comparing the standard deviation, RMSE, and the MSE between the roll and pitch it can be seen that both tend to produce the same level of accuracy.
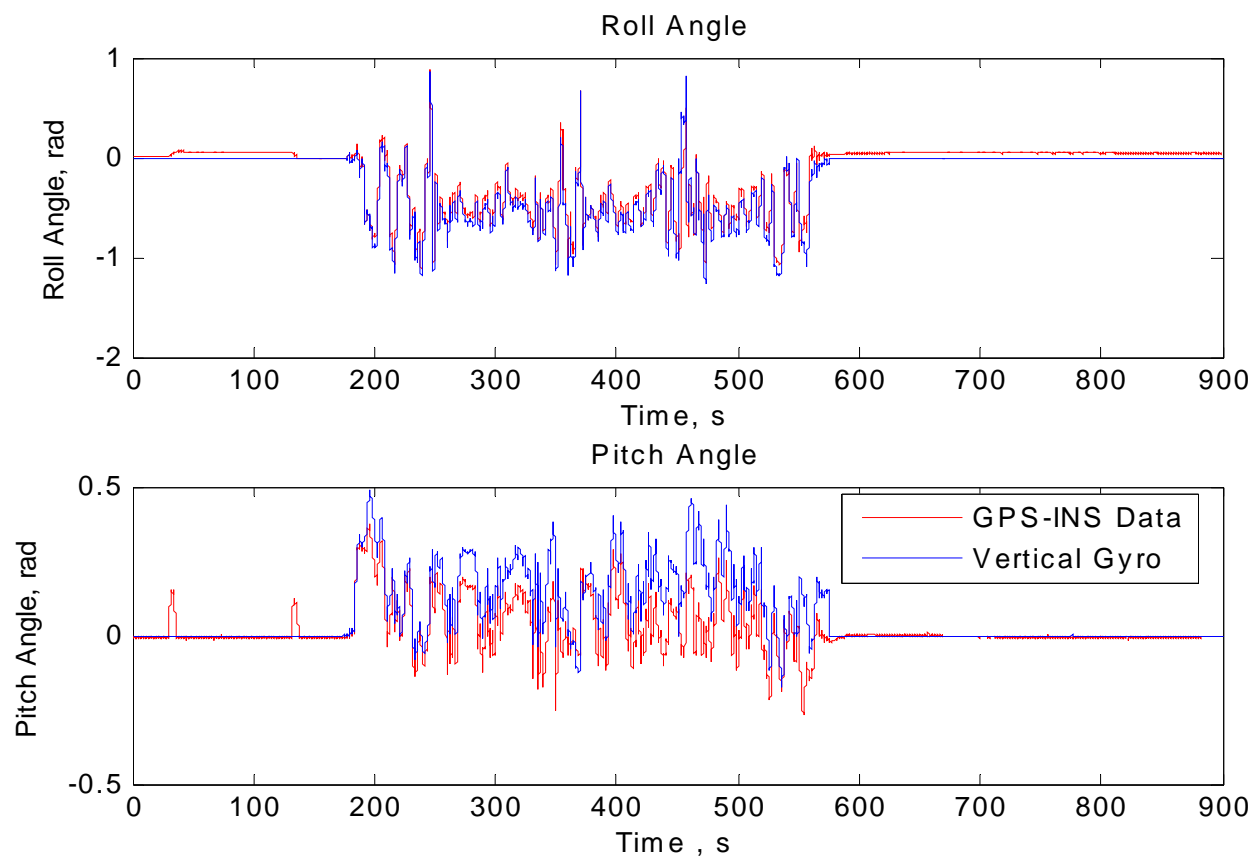

Figure 5.30: GPS-INS Data/Vertical Gyro Comparison (Validation Set 2 - Method II)

The roll angle's STD, MSE, and RMSE are 0.0718, 0.0090, and 0.946, while the pitch angle's respective errors are 0.0580, 0.0078, and 0.0884. After inspecting the error values, it can be seen that the pitch actually has less error than the roll angle for this data set. Generally, for this research topic and the data sets evaluated, it can be deduced that the amount of error reduction in the pitch and roll angles is nearly the same, which also holds true between the various methods. 


\subsection{Computational Workload Analysis}

A computational workload analysis is also conducted using the Matlab ${ }^{\circledR}$ Profiler function, which determines the amount of time spent in each function along with the number of times each function has been executed. The Profiler function helps to also optimize the computer code by pointing out areas with relatively high computation times, which in some cases can be written in a more efficient manner, freeing up additional system resources.

This analysis is conducted for each method so that it can be seen how much of a burden the filter places on the fight computer. The analysis has been divided into the following sections for evaluation:

1. Data Pre-processing

2. GPS Attitude Determination

3. Prediction Equations

a. Estimated State Update

b. State Transition Matrix Update

c. a priori Error Covariance Update

4. Update Equations

a. Kalman Gain Determination

b. Update State Estimate

c. a posteriori Error Covariance Update

Table 5-11 presents the results from the workload analysis so that it can be determined where the majority of the resources are absorbed. For example, by comparing the amount of time the simulation spends in each individual calculation of the filtering process, it can be seen that calculating the Kalman gain in all of the methods takes the most computation time, which is where the highest strain on the system's resources lies. There is two ways of reducing the amount of computational load for each function, first by removing or simplifying the calculation itself, or in some cases the code can be optimized to run at a higher level of efficiency. By revisiting the Kalman gain calculation, there is no additional simplification or code optimization can be conducted leaving the determination of the Kalman gain the most stringent on the system. 
The results in the table are obtained from simulations of the initial data set during the dynamic portion of the WVU YF-22 formation flight. The dynamic flight period was 730 seconds in which the filtering algorithm was executed 14,600 times and the INS was executed 29,200 times, which was due to the GPS and IMU data obtained at $20 \mathrm{~Hz}$ and $40 \mathrm{~Hz}$ respectively. By evaluating the results, several comparisons can be seen from the data, in which the first discusses the differences between the various software methods. The data pre-processing and GPS attitude algorithms are exactly the same for each method (with the exception of method III, which does not have any GPS attitude determination), so the difference in the simulation time is attributed to the number of processes being conducted by the operating system, Windows ${ }^{\circledR}$. The remaining functions simulation times vary due to the size of the matrix $(9 \times 9$ or $2 \times 2)$ and the level of complexity of the calculations being evaluated. Since method III and IV are more complex in terms of the state transition matrix update, their update time increases.

By evaluating and contrasting each method's total simulation time, it can be determined which method is optimal in terms of workload. From Table 5-11, is clearly evident that method II (two-state) has the fastest total simulation time of 2.84 seconds, which can be attributed to the fact that it is only a $2 \times 2$ system. Although this method was the least stringent in terms of computational load, when referring back to the previous section, this method did not post the highest level of error reduction. The nine-state filter produced the best results in terms of error reduction; however the simulation time was 4.65 seconds, which is $63 \%$ slower than the two-state filter. 
Table 5-11: Computational Workload Analysis

\begin{tabular}{|c|c|c|c|c|c|c|c|c|c|c|c|c|c|}
\hline & & \multicolumn{3}{|c|}{ Method II (9-State) } & \multicolumn{3}{|c|}{ Method II (2-State) } & \multicolumn{3}{|c|}{ Method III } & \multicolumn{3}{|c|}{ Method IV } \\
\hline & & $\begin{array}{l}\text { Time } \\
\text { (sec) }\end{array}$ & Execution & $\begin{array}{c}\text { Time/ } \\
\text { Execution } \\
\text { (sec) }\end{array}$ & $\begin{array}{l}\text { Time } \\
\text { (sec) }\end{array}$ & Executions & $\begin{array}{c}\text { Time/ } \\
\text { Execution } \\
\text { (sec) }\end{array}$ & $\begin{array}{l}\text { Time } \\
\text { (sec) }\end{array}$ & Executions & $\begin{array}{c}\text { Time/ } \\
\text { Execution } \\
\text { (sec) } \\
\end{array}$ & $\begin{array}{l}\text { Time } \\
\text { (sec) }\end{array}$ & Executions & $\begin{array}{c}\text { Time/ } \\
\text { Execution } \\
\text { (sec) }\end{array}$ \\
\hline \multicolumn{2}{|c|}{ Data Preprocessing } & 0.176 & 1 & N/A & 0.176 & 1 & N/A & 0.187 & 1 & N/A & 0.179 & 1 & $\mathrm{~N} / \mathrm{A}$ \\
\hline \multicolumn{2}{|c|}{$\begin{array}{c}\text { GPS Attitude } \\
\text { Determination }\end{array}$} & 0.583 & 14600 & $3.99 \mathrm{e}^{-5}$ & 0.548 & 14600 & $3.75 \mathrm{e}^{-5}$ & N/A & N/A & N/A & 0.619 & 14600 & $4.23 \mathrm{e}^{-5}$ \\
\hline \multirow{3}{*}{$\begin{array}{l}\text { Prediction } \\
\text { Equations }\end{array}$} & $\begin{array}{c}\text { Estimated } \\
\text { State } \\
\text { Update } \\
\end{array}$ & 0.777 & 29200 & $2.66 \mathrm{e}^{-5}$ & 0.360 & 29200 & $1.23 \mathrm{e}^{-5}$ & 0.757 & 29200 & $2.59 \mathrm{e}^{-5}$ & 0.768 & 29200 & $2.63 \mathrm{e}^{-5}$ \\
\hline & $\begin{array}{c}\text { STM } \\
\text { Update } \\
\end{array}$ & 0.383 & 14600 & $2.62 \mathrm{e}^{-5}$ & 0.235 & 14600 & $1.61 \mathrm{e}^{-5}$ & 0.522 & 14600 & $3.58 \mathrm{e}^{-5}$ & 0.517 & 14600 & $3.54 \mathrm{e}^{-5}$ \\
\hline & $\begin{array}{c}\text { a priori } \\
\text { Error } \\
\text { Covariance } \\
\text { Update }\end{array}$ & 0.414 & 14600 & $2.83 \mathrm{e}^{-5}$ & 0.226 & 14600 & $1.55 \mathrm{e}^{-5}$ & 0.412 & 14600 & $2.82 \mathrm{e}^{-5}$ & 0.346 & 14600 & $2.37 \mathrm{e}^{-5}$ \\
\hline \multirow{3}{*}{$\begin{array}{c}\text { Update } \\
\text { Equations }\end{array}$} & $\begin{array}{c}\text { Kalman } \\
\text { Gain }\end{array}$ & 1.194 & 14600 & $8.18 \mathrm{e}^{-5}$ & 0.656 & 14600 & $4.49 \mathrm{e}^{-5}$ & 0.911 & 14600 & $6.24 \mathrm{e}^{-5}$ & 1.132 & 14600 & $7.75 \mathrm{e}^{-5}$ \\
\hline & $\begin{array}{l}\text { Update } \\
\text { State }\end{array}$ & 0.742 & 14600 & $5.08 \mathrm{e}^{-5}$ & 0.390 & 14600 & $2.67 \mathrm{e}^{-5}$ & 0.631 & 14600 & $4.32 \mathrm{e}^{-5}$ & 0.804 & 14600 & $5.51 \mathrm{e}^{-5}$ \\
\hline & $\begin{array}{c}\text { a posteriori } \\
\text { Error } \\
\text { Covariance } \\
\text { Update } \\
\end{array}$ & 0.387 & 14600 & $2.65 \mathrm{e}^{-5}$ & 0.250 & 14600 & $1.71 \mathrm{e}^{-5}$ & 0.361 & 14600 & $2.47 \mathrm{e}^{-5}$ & 0.385 & 14600 & $2.64 \mathrm{e}^{-5}$ \\
\hline \multicolumn{2}{|c|}{ Total Time } & 4.65 & N/A & N/A & 2.84 & N/A & N/A & 3.78 & N/A & N/A & 4.75 & N/A & N/A \\
\hline
\end{tabular}


Of the nine-state filters (Methods II, III, and IV), method III is the fastest, but yet again, it's the worst performing filter in terms of error reduction. While methods II (nine-state) and IV are the best performing in terms of error reduction, they both post simulation times of 4.65 and 4.75 seconds, which are the worst overall. The additional time can be attributed to the calculation of the GPS estimated attitudes, which is not calculated in Method III.

At this point, when designing a control system, one must take into account the amount of computational resources available and select the best fit for the application. By comparing the computational workload analysis with the error analysis in the previous section, it is evident that the best results are proportional to the amount of computational resources available. 


\section{Chapter 6. Conclusions and Recommendations}

\subsection{Conclusions}

The ability to obtain higher precision data with lower cost, smaller sensor packages is becoming a standard not only in UAV and MAV research, but also all areas of guidance and navigation, including commercial, civilian, and military full scale applications. Although, while sensor fusion is becoming easier to implement, not only due to the fact that vehicle computers are able to handle higher levels of computation, but also for the need to become smaller, expendable, and power conscious depending on the application.

The results of this effort show that the integration of INS/GPS sensors is a sufficient, low-cost solution for position, velocity, and attitude determination in which the sensor fusion methods presented here exhibited satisfactory results and should be considered for implementation for any system in which a higher level of precision is required when only a low-cost sensor platform is available. Simulation results from the real-world flight data prove that each of the filtering methods presented throughout this report reduced the amount of error in the navigation system. As discussed in Section 5.6, the nine-state filter from method II produced the highest level of error reduction over the four data sets use., The results from the second method could be associated with a number of issues; first, since the EKF is not a true "optimal" filter, it is more susceptible to divergence and residual error if the system is not initially set-up/modeled correctly. These initial setup errors would mainly be tied with the calculation of the model/input and measurement covariance matrix that determines the amount of correction to be adjusted back into the state estimate. The linearization of the aircraft kinematic equations and direction cosine matrix produce errors due to the fact that the higher order terms are neglected leaving residual errors in the system not modeled or taken into consideration which is the reason for the filter not being optimal. Method II produces better results due to the fact that the direction cosine matrix is not linearized and inserted into the state transitions matrix as in methods III and IV. 
It is also worthwhile to restate that the implementation in the second method, all nine states were not required to produce adequate results, in which the system state matrix can be reduced to as little as two states (pitch and roll) and still produce equivalent results in regards to system performance while greatly reducing the computational workload. Although this cannot be done for the third method for it utilizes the position and velocity for the correction of the attitude. This would be especially important in MAV's when computation power is limited.

\subsection{Recommendations}

The future of sensor fusion applications within the field of guidance and navigation will certainly continue to become more widespread as the need for smaller, cheaper UAV's and MAV's become the norm in the military and civilian world. To further build on the research presented within the context of this thesis, it is recommended to implement these methods in a real-time environment as to test the robustness of the system in determining attitude, position, and velocity data in regards to aircraft control as well as the computational load on the flight computer.

In regards to recommendations for the research conducted within the context of this research project, additional means in reducing error generated from the linearization errors and covariance analysis could also reduce the amount of error in the EKF. The sampling rate between the GPS $(20 \mathrm{~Hz})$ and the IMU $(40 \mathrm{~Hz})$ also plays a role in the efficiency of the filter; meaning that if the GPS and IMU were to be sampled at the same frequency the system would have less error, with the drawback of having a lower sampling rate. Testing to find the optimal sampling rates may also help to decrease error in the system.

The method for determining the covariance matrices is also an area that could increase the performance of the filtering process. Since the covariance's are determined from the sensors during a steady-state period, the exact values could be skewed due to inaccuracies of the data. By more accurately determining the actual covariance's of the sensors, the filter would produce better results, especially for the EKF.

At the time this thesis was written, researches at WVU have already began conducting research on two separate programs requiring the implementation of these 
sensor fusion methods for the determination of aircraft attitude for use in control schemes. The first is to utilize sensor fusion methods in a low-cost miniature autopilot capable of being implemented on commercial off-the-shelf $r / c$ aircraft at a minimum expense $^{51}$. The miniature autopilot does not have the benefit of having a vertical gyro to obtain highly precise attitude angles, however it is equipped with both GPS and IMU sensors that allow for sensor fusion implementation.

The second project is for a total redesign of the WVU YF-22 test vehicles onboard-computer in which various sensor fusion schemes will be tested in a real-time flight environment. 


\section{References}

1 Seanor, B., Campa, G., Gu, Y., Napolitano, M.R., Rowe, L., Perhinschi,M. Formation Flight Test Results for UAV Research Aircraft Models", Proceedingsof the 2004 AIAA Intelligent Systems Technology Conference, Chicago, IL, September 2004.

2 Napolitano, M.R.,: Development of formation flight control algorithms using 3 YF-22 flying models. Final Report, Air Force Office of Scientific Research, AFOSR Grant Number F49620-01-1-0373 (2005)

3 "The Wiener Filter" University of Waterloo - Canada Jan 1998. http://ubiety.uwaterloo.ca/ tveldhui/papers/MAScThesis/node12.html

4 Masani, P. R., Norbert Wiener, 1894-1964. Birkhauser, 1990.

5 Kalman, R.E. (1960). "A new approach to linear filtering and prediction problems". Journal of Basic Engineering 82 (1): 35-45.

6 Lewis, F.L. and Sam Ge, Shuzhi, Autonomous Mobile Robots, Taylor and Francis, New York, 2006.

$7 \quad$ Kalman, R.E.; Bucy, R.S. (1961). "New Results in Linear Filtering and Prediction Theory

8 Dunbar, Dino, NASA Ames Research Labs. "History Related to the Apollo Moon Program and Lunar Prospector Mission April 21, 2007.http://www.nasa.gov/lb/centers/ames/news/releases/2004/moon/apollo_ames_atmos .html

9 Karlsson, Mia. "Non-linear Dynamic Inversion." Thesis Linkoping University, 2002

10 Lewis, F. and Syrmos, V. Optimal Control. John Wiley and Sons: New York, NY, 1995.

11 S. J. Julier and J. K. Uhlmann. "A New Extension of the Kalman Filter to Nonlinear Systems." In The Proceedings of AeroSense: The 11th International Symposium on Aerospace/Defense Sensing, Simulation and Controls, Orlando, FL, USA, 1997. SPIE. Multi Sensor Fusion, Tracking and Resource Management II. 
12 "A Short History of Sperry Marine" Northrop Grumman. http://www.sperrymarine.northropgrumman.com/Company-formation/CorporateHistory/Sperry-History/

13 "Navy of the Future". U.S. Navy. http://www.navy.com/about/shipsequipment/navyofthefuture/

14 Kagey, Price. "Sensor Fusion: Cuts Through the Clutter." Lockheed Martin: Insights Online. 2006. http://www.lockheedmartin.com/data/assets/13551.pdf

15 Wells, Brian. "Systems Architecture." Northrop Grumman: technology today.Summer 2003, Volume 2. http://www.raytheon.com/technology_today/archive/ 2003_Issue2.pdf

16 Mchale, Tom. "Integrated Undersea Warfare System." Northrop Grumman: technology today. Summer 2003, http://www.raytheon.com/technology_today/archive/2003_Issue2.pdf

17 Bennet, Stewart. A History of Control Engineering, 1800-1930. Stevenage, UK:Peter Peregrines, 1979.

18 Myschik, S., Holzapel, F., and Sachs, G. "Low-Cost Sensor Based Integrated Airdata - and Navigation System for General Aviation Aircraft.", AIAA Hawaii, August 2008.

19 "Boeing Unmanned Little Bird Demonstrator Helicopter Flies Unmanned for First Time." Boeing - News Release. July 12, 2006. http://www.boeing.com/ids/news/2006/q3/060712c_nr.html

20 Ford, T., Hardesty, M., Bobye, M., "Helicopter Ship Board Landing System."

21 Ettinger, S. M., Nechyba, M.C., Ifju, P., Wasak, M. "Vision Guided Stability andControl for Micro Air Vehicles." Proc. IEEE Int. Conference on Intelligent Robots and Systems, October, 2002

22 Mammarella, Marco, Giampiero Campa, Marcello Napolitano, M. Fravolini, Yu $\mathrm{Gu}, \mathrm{M}$. Perinschi. "Machine Vision/GPS Integration Using EKF for the UAV Aerial Refueling Problem." IEEE Transactions on Systems, Manned, and Cybernetics. To be published in 2008.

23 Clemens, R., Pedro Tavares, Pedro Lima. "Small Satellite Attitude Control Based on a Kalman Filter." Proceedings of the IEEE International Symposium on Intelligent Control. 2000. Patras, Greece. 
24 Sethia, Vikash. "Noise Canceling Headphones." M.Tech. credit seminar report, Electronic Systems Group, EE Dept, IIT Bombay, November 2002

25 Oppenheim, A. V., Weinstein, E., Zangi, K.C., Feder, M., and Gauger, D., Single Sensor Active Noise Cancellation Based on the EM Algorithm." IEEE International Conference on HAcoustics, Speech, and Signal ProcessingH, 23-26 March, 1992.

26 "Report to Congress, DARPA Prize Authority" Defense Advanced Research Projects Agency, March 2006

27 Mudaliar, Nikhil; Leblanc, David ; Peng, Huei . "Linear estimator for road departure warning systems." Proceedings of the American Control Conference, v 3, Proceedings of the 2004 American Control Conference (AAC), 2004, p 2104-2109

28 "MEMS IMU" empfasis. 2003. http://www.empf.org/empfasis/oct03/603memsimu.htm.

29 "EX-171 ERGM Extended-Range Munition" Global Security. July 2005. http://www.globalsecurity.org/military/systems/munitions/ergm.htm

30 "Manufacturing Technology for MEMS-based IMUs" Office of Naval Research. July 2005. http://www.onr.navy.mil/sci_tech/3t/mantech/docs/success_stories/ships/ships IMUs_121505.pdf.

31 "XM982 Excalibur Precision Guided Extended Range Artillery Projectile" Global Security. July 2005. http://www.globalsecurity.org/military/systems/munitions/m982155.htm

32 J.Yin, V.L.Syrmos and D.Y .Y .Yun. "System Identification using the Extended Kalman Filter with Applications to Medical Imaging." Proceedings of the American Control Conference. Chicago, Il June 2000.

33 W. Wu, M. Black D. Mumford, Y. Gao, E. Bienenstock, and J. Donoghue, "Modeling and Decoding Motor Cortical Activity Using a Switching Kalman Filter," IEEE Transactions On Biomedical Engineering, VOL. 51, NO. 6, June 2004.

34 S. H.S. Helms Tillery, D. Taylor, R. Isaacs, and A. Schwartz, "Online control of a prosthetic arm from motor cortical signals," Soc. Neurosci. Abst., vol. 26, 2000. 
35 M. D. Serruya, N. G. Hatsopoulos, L. Paninski, M. R. Fellows, and J. P.Donoghue, "Brain-machine interface: instant neural control of a movement signal," Nature, vol. 416, pp. 141-142, 2002.

36 A. Tzallas, V. Oikonomou, and D. Fotiadis, "Epileptic Spike Detection Usinga Kalman Filter Based Approach," Proceedings of the 28th IEEE EMBS Annual International Conference, New York City, USA, Aug 30-Sept 3, 2006.

37 Johnson, Lorne D. and Sakoulis, Georgios, "Maximizing Equity Market Sector Predictability in a Bayesian Time Varying Parameter Model" (July 2003).

38 Harvey, Andrew, Forecasting, Structural Time Series Models and the Kalman Filter. Cambridge University Press: New York, NY 1990

39 Xiaoyun, L, Xiaoling, Z., Xian, X, "Application of Kalman Filter for Agriculture Economic Forecasting." Proceedings from the Systems, Man, and Cybernetics IEEE International Conference, 1996

40 Farrell, Jay, Barth, Matthew. The Global Positioning System and Inertial Navigation. McGraw-Hill: New York, NY, 1999.

41 Brittling, K.R., Inertial Navigation Systems Analysis, Wiley-Interscience, New York, 1971

42 Farrell, J.L., Integrated Aircraft Navigation, Academic, New York, 1976

43 “Aerospace Blockset." http://www.mathworks.com/access/helpdesk/help/toolbox/ aeroblks/index.html?/access/helpdesk/help/toolbox/aeroblks/f322568.html\&http://www.g oogle.com/search? sourceid=navclient\&ie $=$ UTF-8\&rlz=1T4RNWO_enUS210US210\&q= matlab $\% 2 \mathrm{c}+$ earth + centered + inertial + frame

44 Parkinson B., Spilker J., Axelrad, P., Enge, P., Global Positioning System: Theory and Applications, AIAA, 1996

45 Kaplan, E.D.ed., Understanding GPS: Principles and Application, Boston: Artec House, c1996.

46 Diebel, J. "Representing Attitude: Euler Angles, Unit Quaternions, and RotationVectors." Stanford University, October 2006.

47 "Geodetic System." Wikipedia The Free Encyclopedia. 5 May 2008, 16:23 http://en.wikipedia.org/wiki/Geodetic_system 
48 “Gimbal.” http://www.mathworks.com/access/helpdesk/help/toolbox/physmod/ mech/ndex.html?/access/helpdesk/help/toolbox/physmod/mech/gimbal.html\&http://imag es.google.com/imgres?imgurl=http://www.mathworks.com/access/helpdesk/help/toolbox/ physmod/mech/gimbal.gif\&imgrefurl=http:/www.mathworks.com/access/helpdesk/help/ toolbox $/ \mathrm{physmod} / \mathrm{mech} / \mathrm{gimbal} . \mathrm{html} \& \mathrm{~h}=516 \& \mathrm{w}=410 \& \mathrm{sz}=42 \&$ tbnid $=7 \mathrm{naqdshWASMeM}$ :\&tbnh $=131 \&$ tbnw $=104 \& \mathrm{hl}=$ en \&prev $=/$ images $\% 3 \mathrm{Fq} \% 3 \mathrm{Dgimbal} \% 26 \mathrm{gbv} \% 3 \mathrm{D} 2 \% 26 \mathrm{hl} \%$ 3Den $\&$ frame $=$ small

49 Lewis, F., Syrmos, and V. Optimal Control, John Wiley and Sons: New York, NY, 1995, pp. 188.

50 D. Kingston and R. Beard: Real Time Attitude Position and Estimation for Small UAVs Using Low-Cost Sensors. AIAA $3^{\text {rd }}$ "Unmanned Unlimited" Technical Conference, WEorkshop and Exhibit, Chicago Ill., Sept. 20-23, (2004)

51 Cukic, B., Gu, Y, Seanor, B.,: D Development of a Small, Ultra Low Cost, and Flexible UAV Test-Bed., NASA West Virginia Space Grant Consortium, (2007) 


\section{Appendix A}

\section{Method II - Additional Plots and Error Analysis}



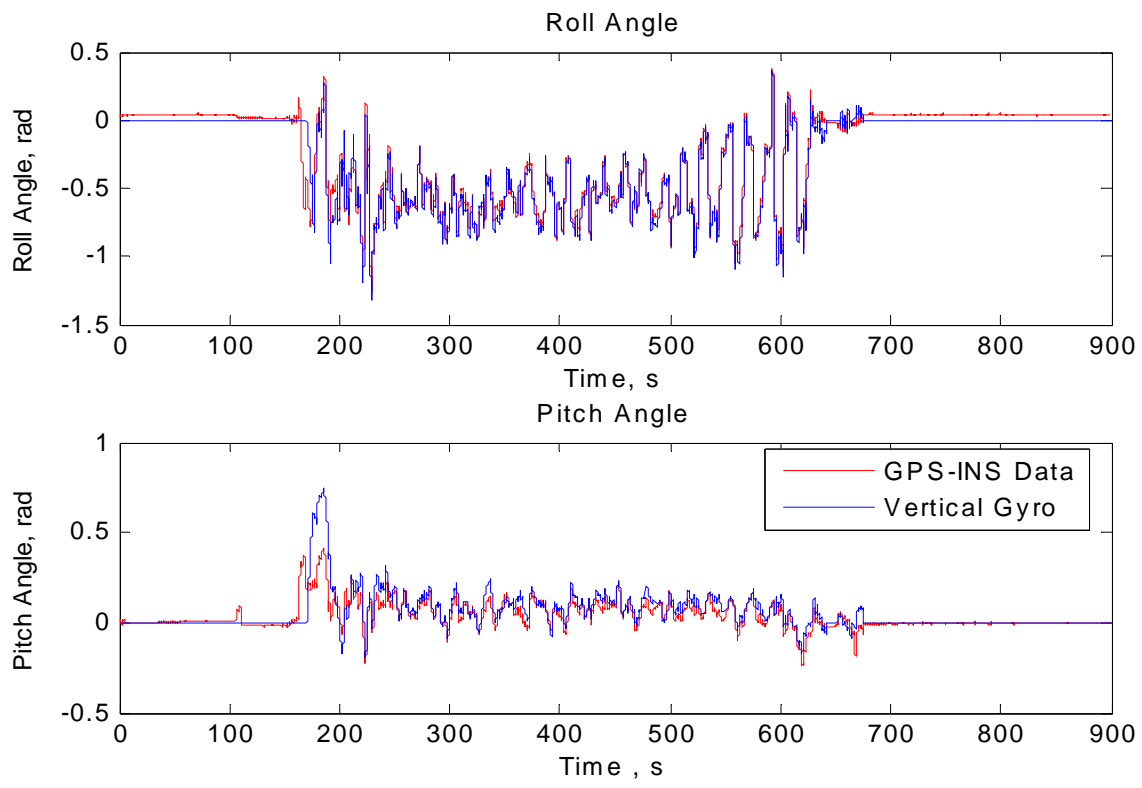

Figure A.1: GPS-INS Data/Vertical Gyro Comparison (Validation Set 1)
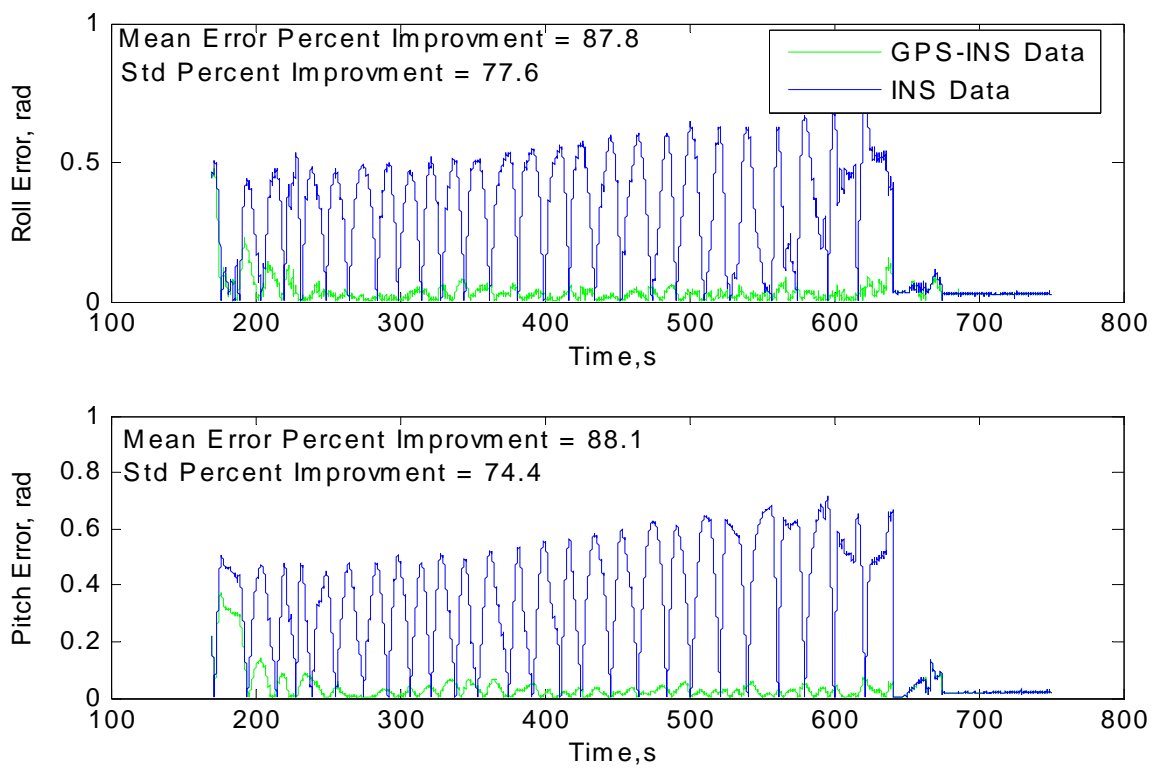

Figure A.2: Two State Roll and Pitch Error (Validation Set 1) 

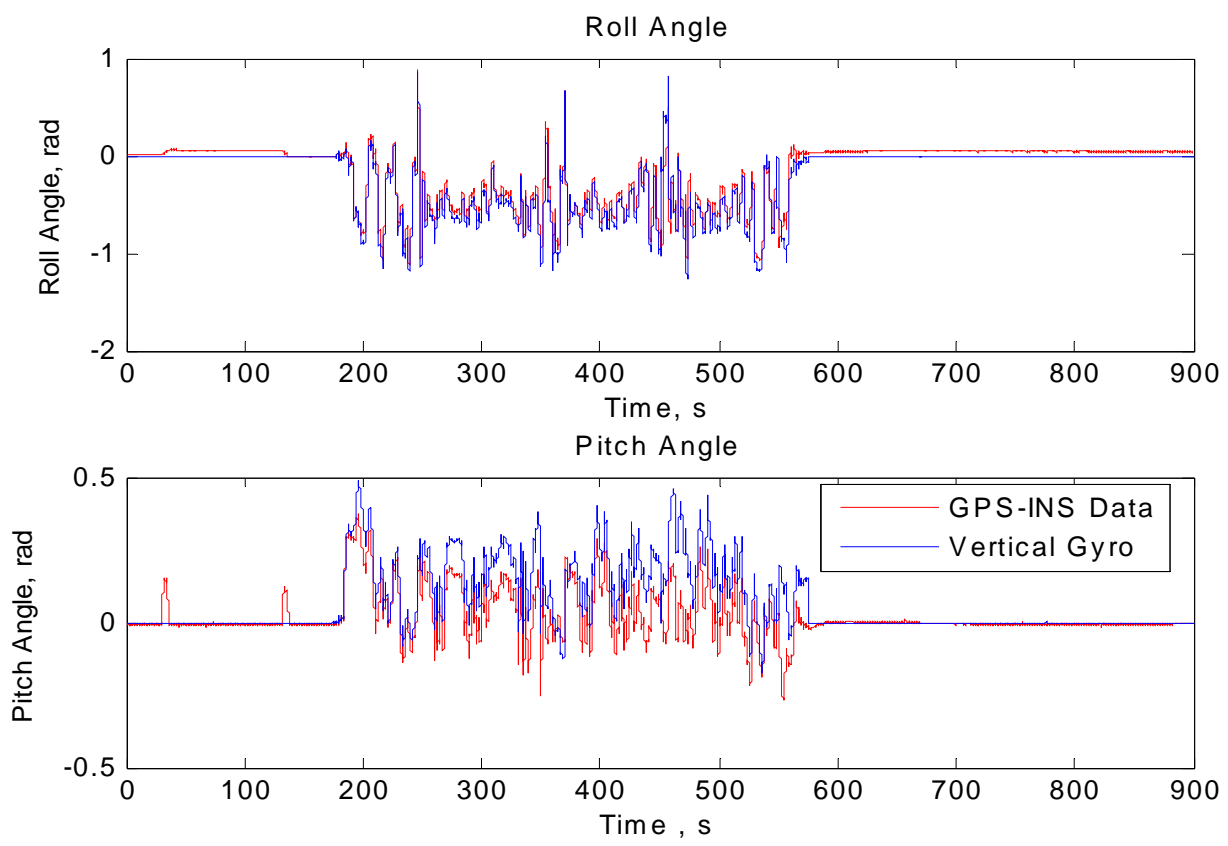

Figure A.3: GPS-INS Data/Vertical Gyro Comparison (Validation Set 2)
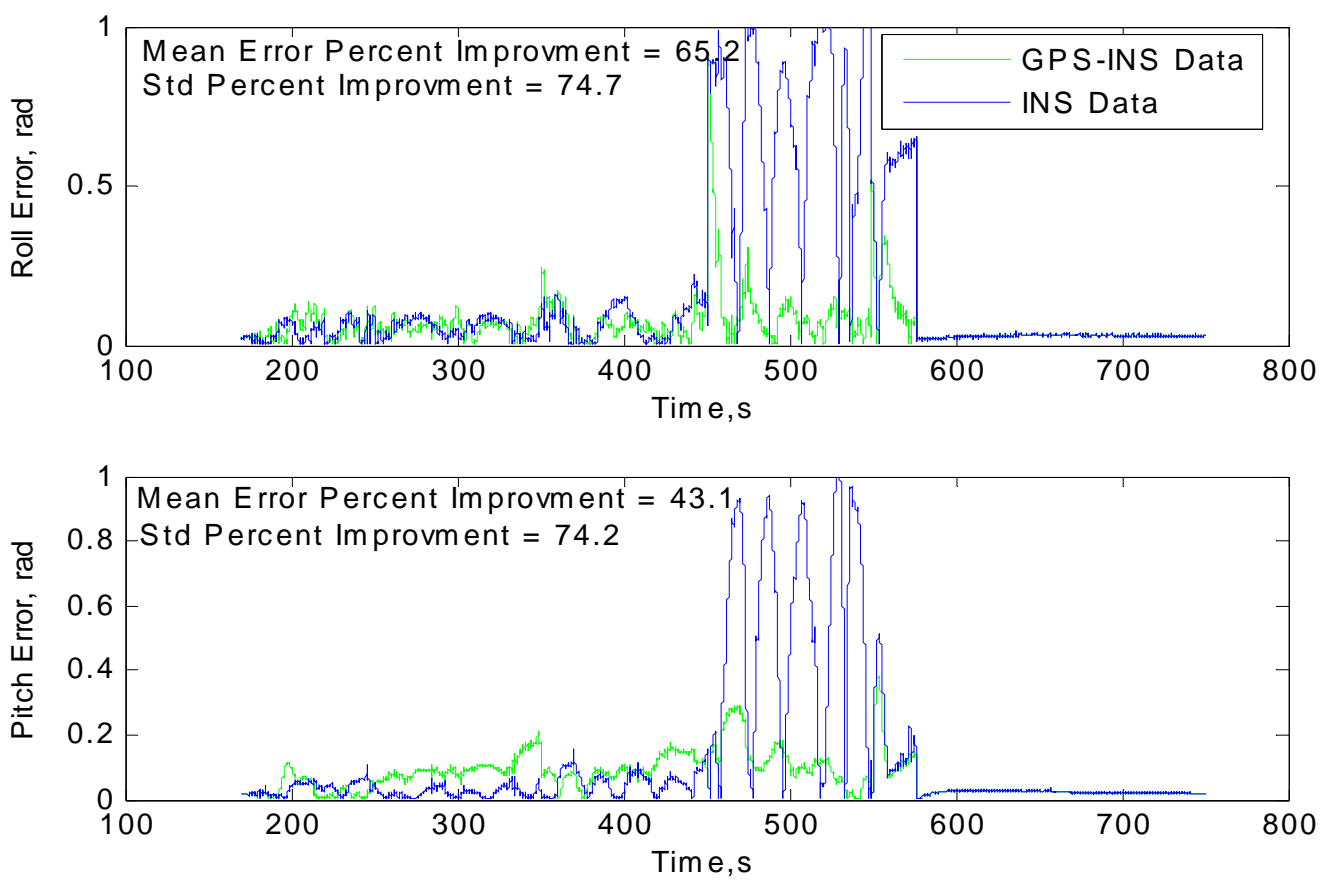

Figure A.4: Two State Roll and Pitch Error (Validation Set 2) 


\section{Appendix B}

Method III - Additional Plots and Error Analysis 

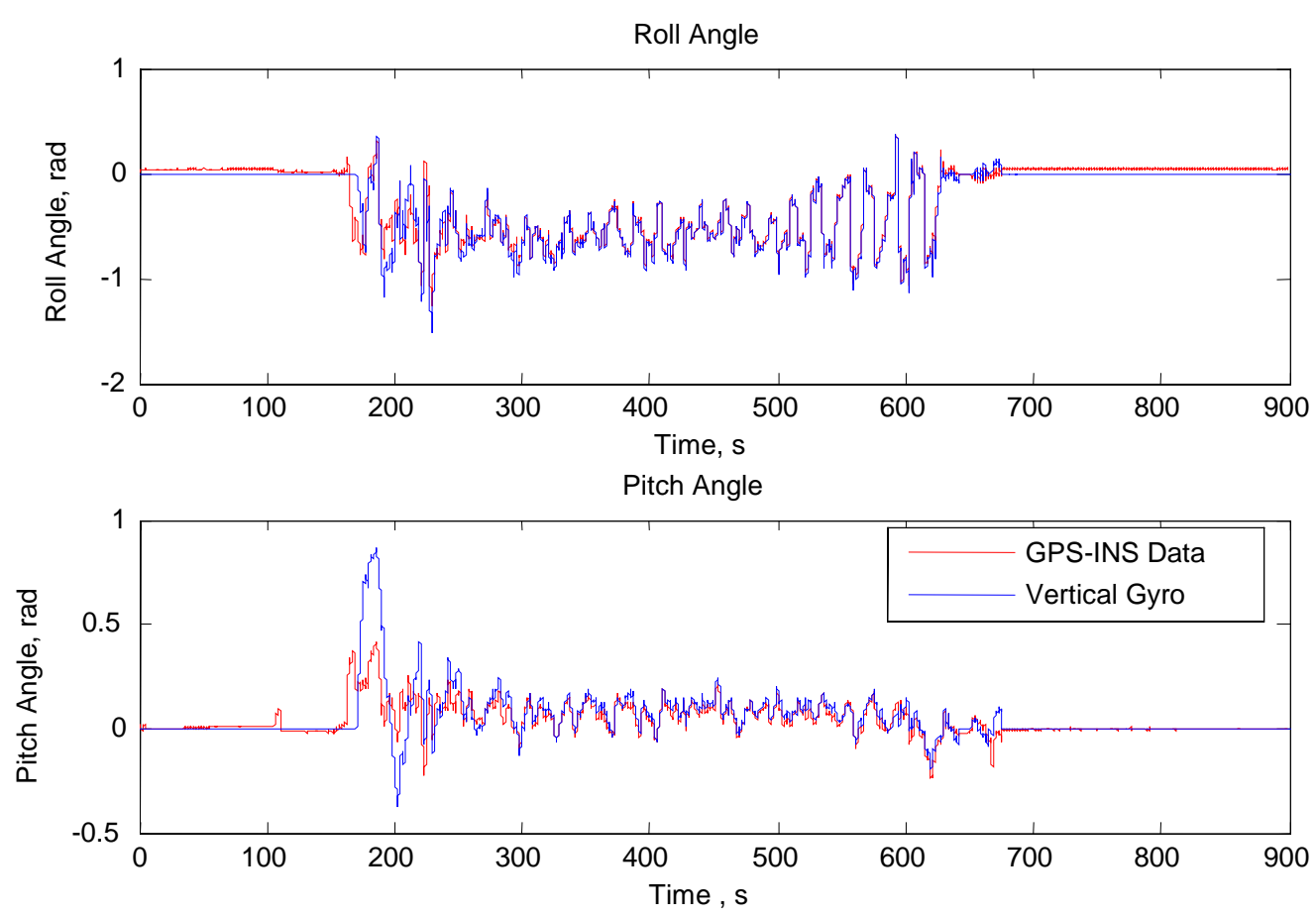

Figure B.1: GPS-INS Data/Vertical Gyro Comparison (Validation Set 1)
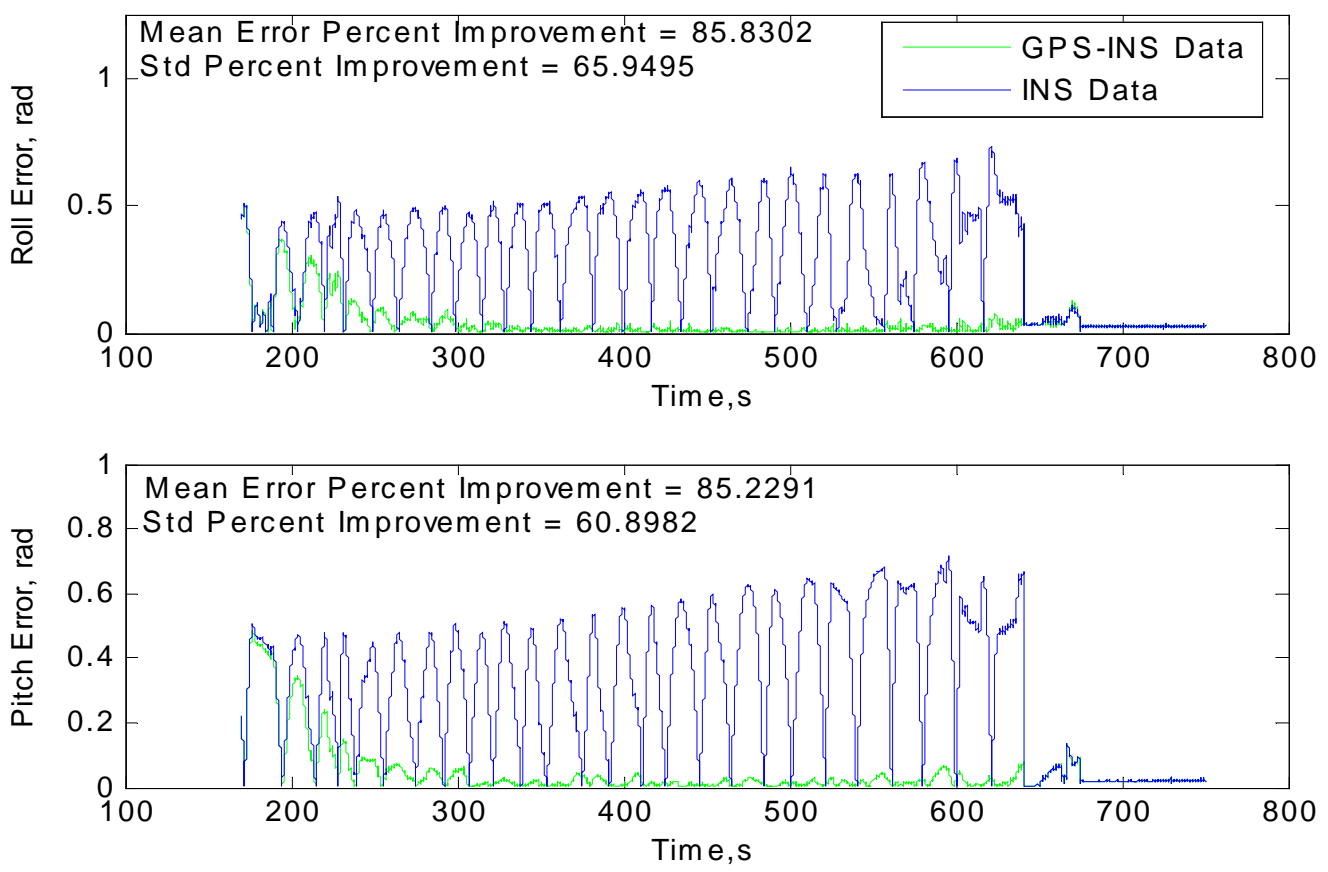

Figure B.2: Method III Roll and Pitch Error (Validation Set 1) 
Roll Angle

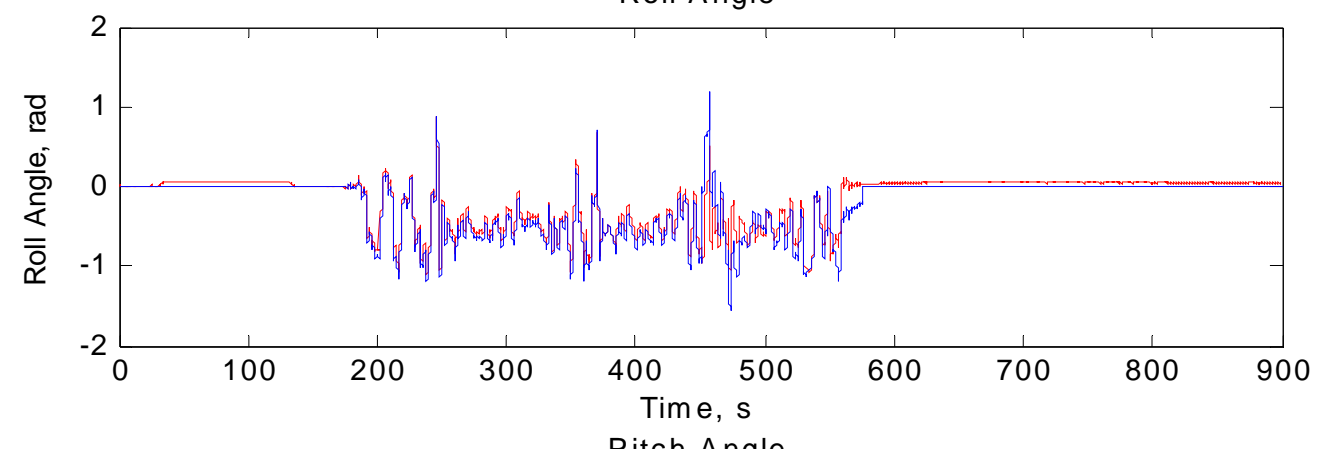

Pitch Angle

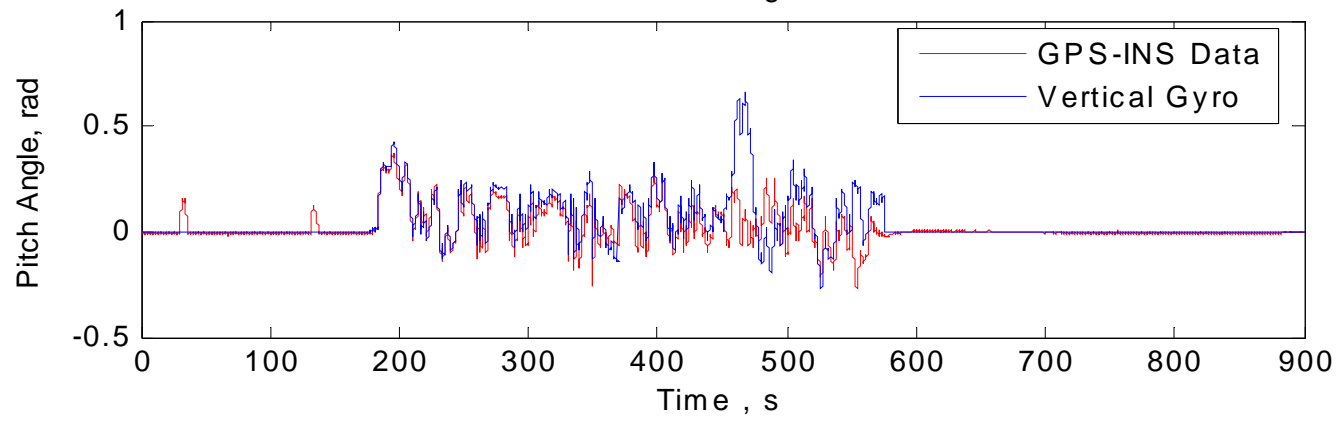

Figure B.3: GPS-INS Data/Vertical Gyro Comparison (Validation Set 2)
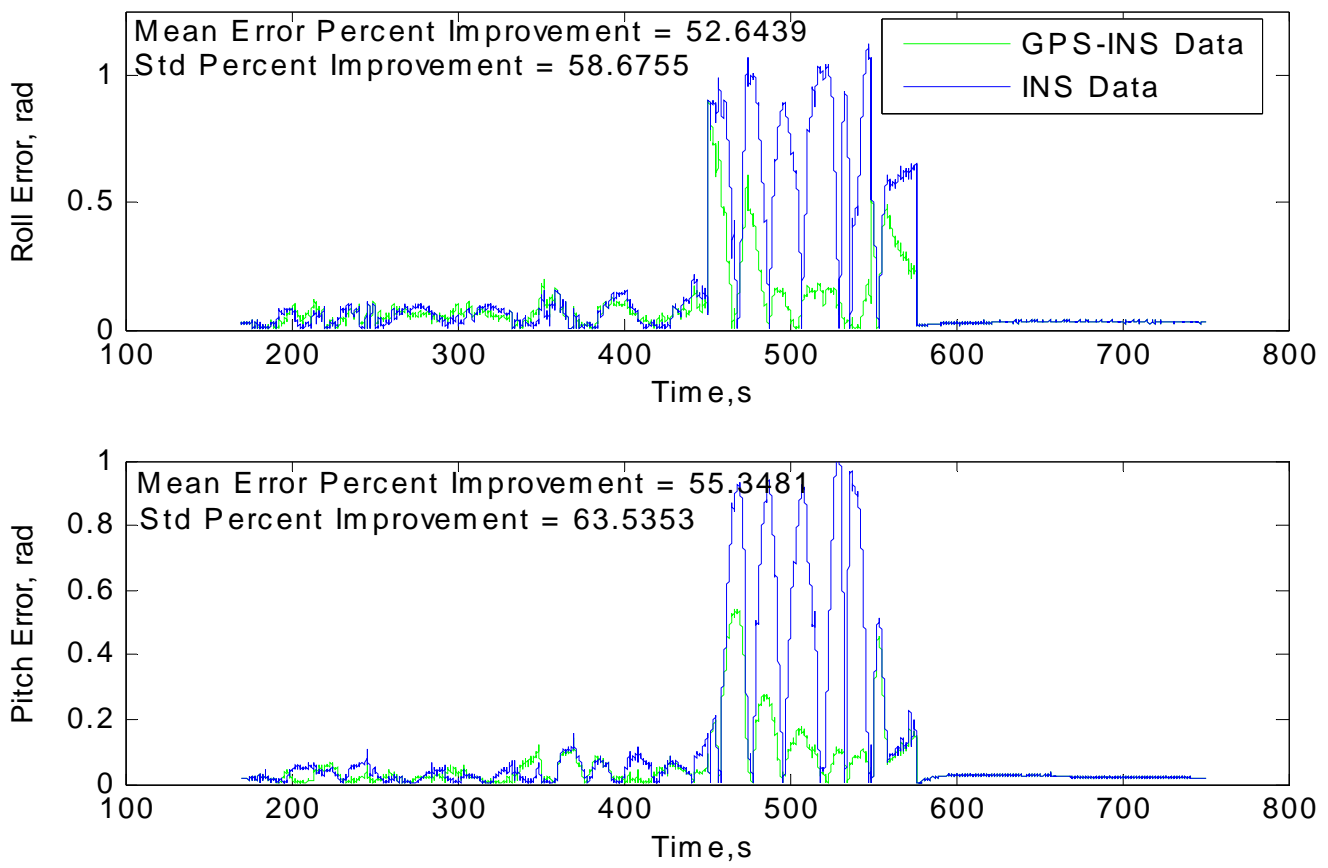

Figure B.4: Method III Roll and Pitch Error (Validation Set 2) 


\section{Appendix C}

Method IV - Additional Plots and Error Analysis 


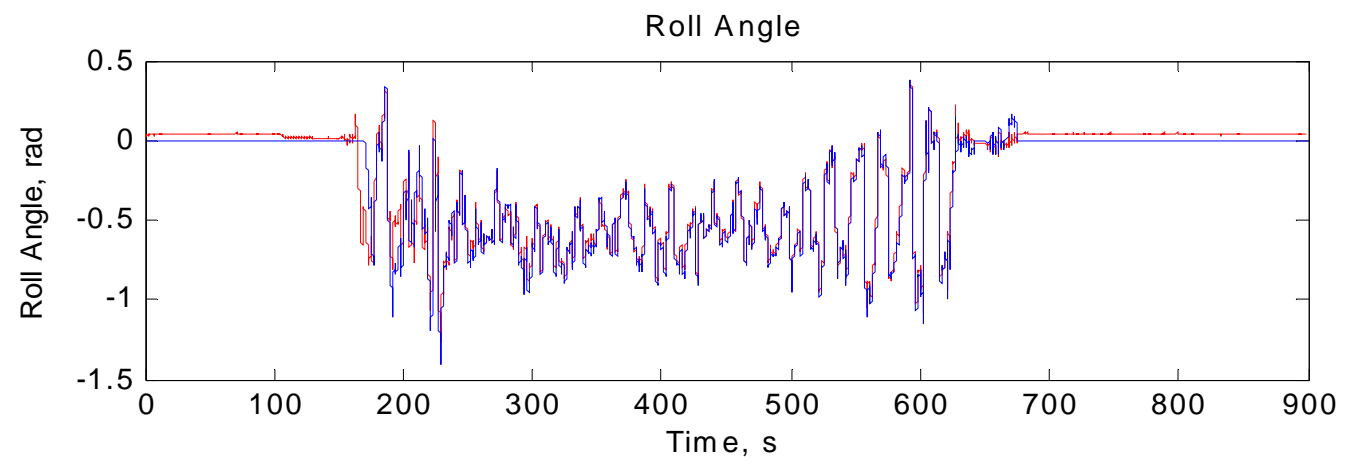

Pitch Angle

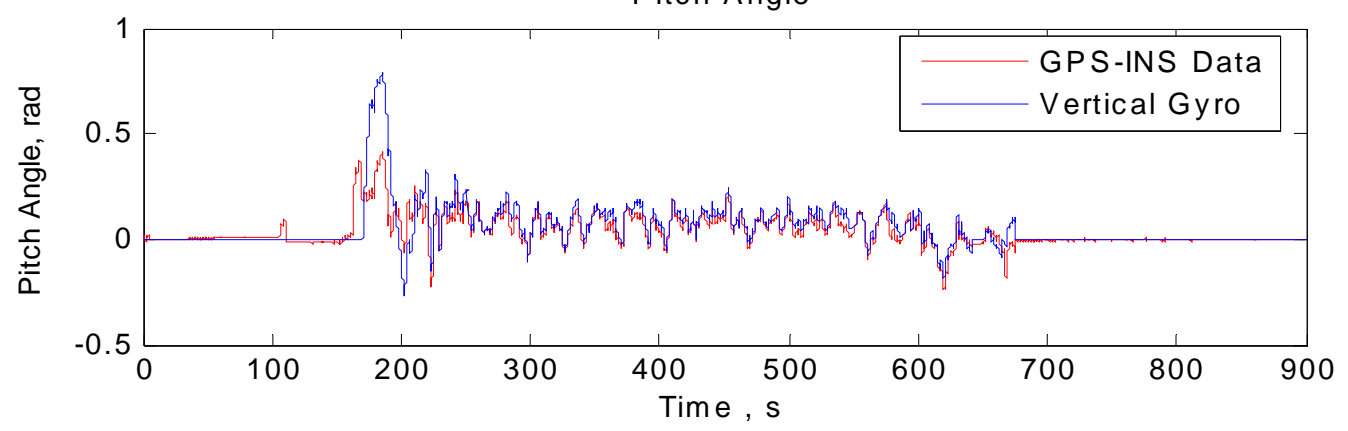

Figure C.1: : GPS-INS Data/Vertical Gyro Comparison (Validation Set 1)
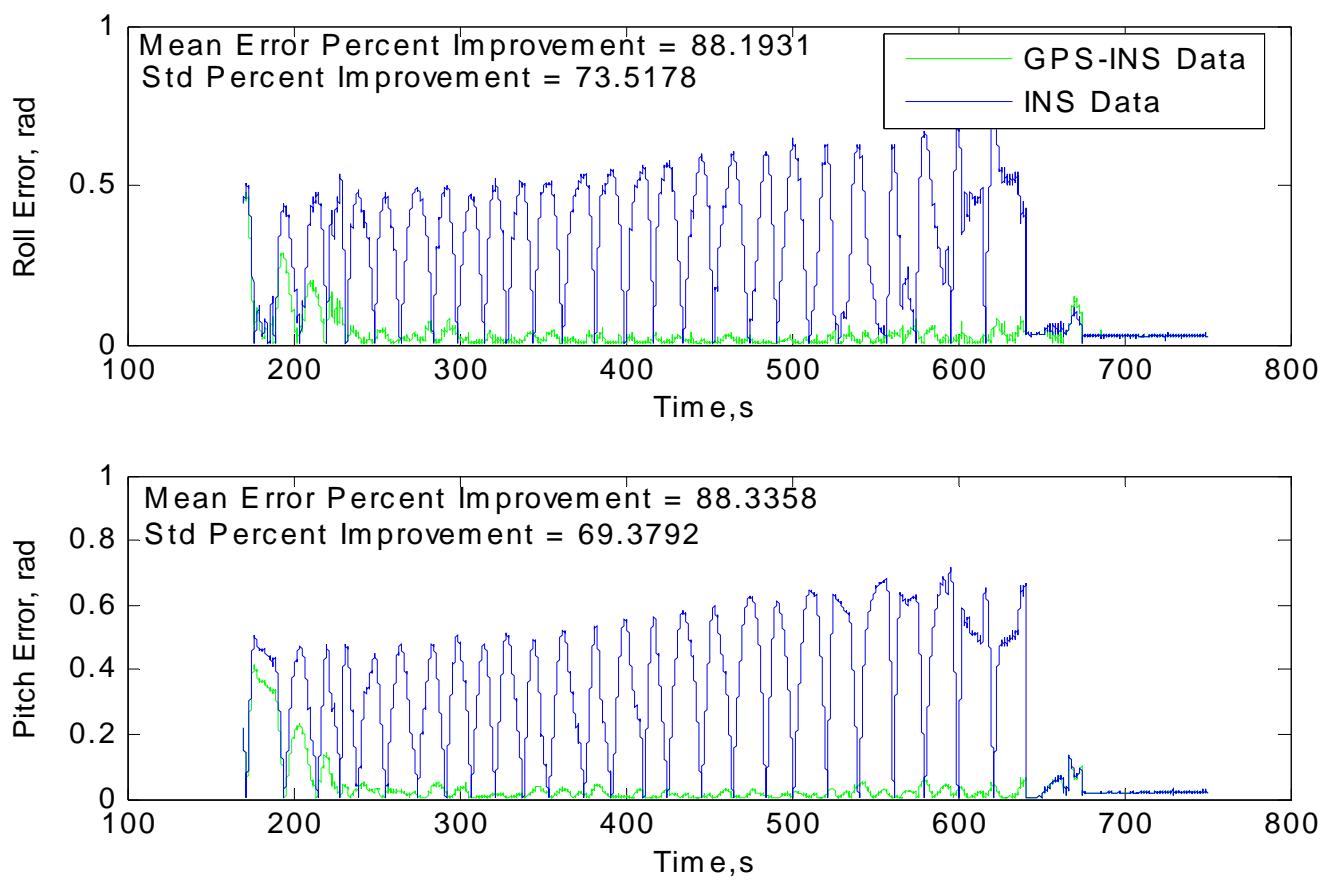

Figure C.2: Method IV Roll and Pitch Error (Validation Set 1) 

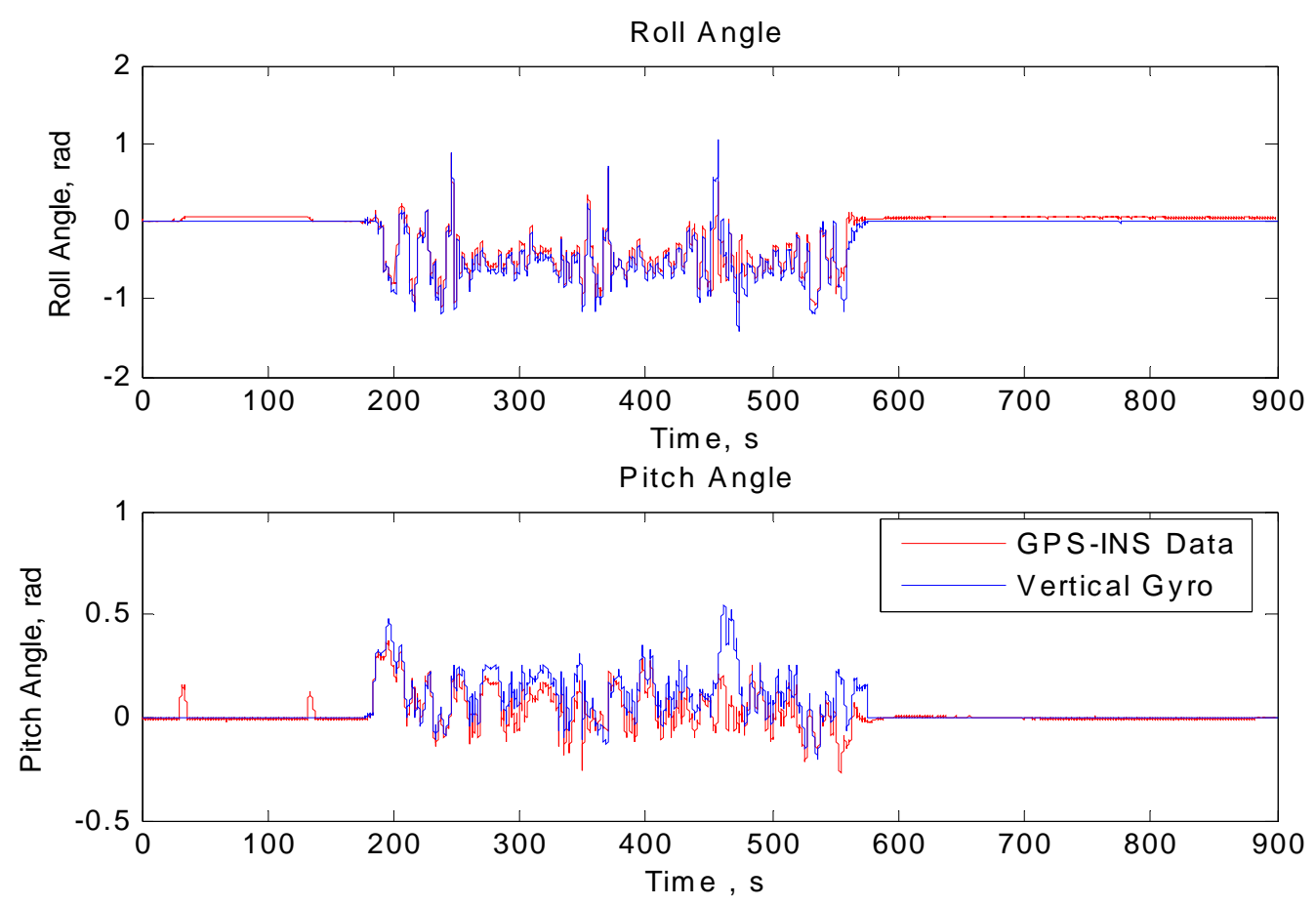

Figure C.3: GPS-INS Data/Vertical Gyro Comparison (Validation Set 2)
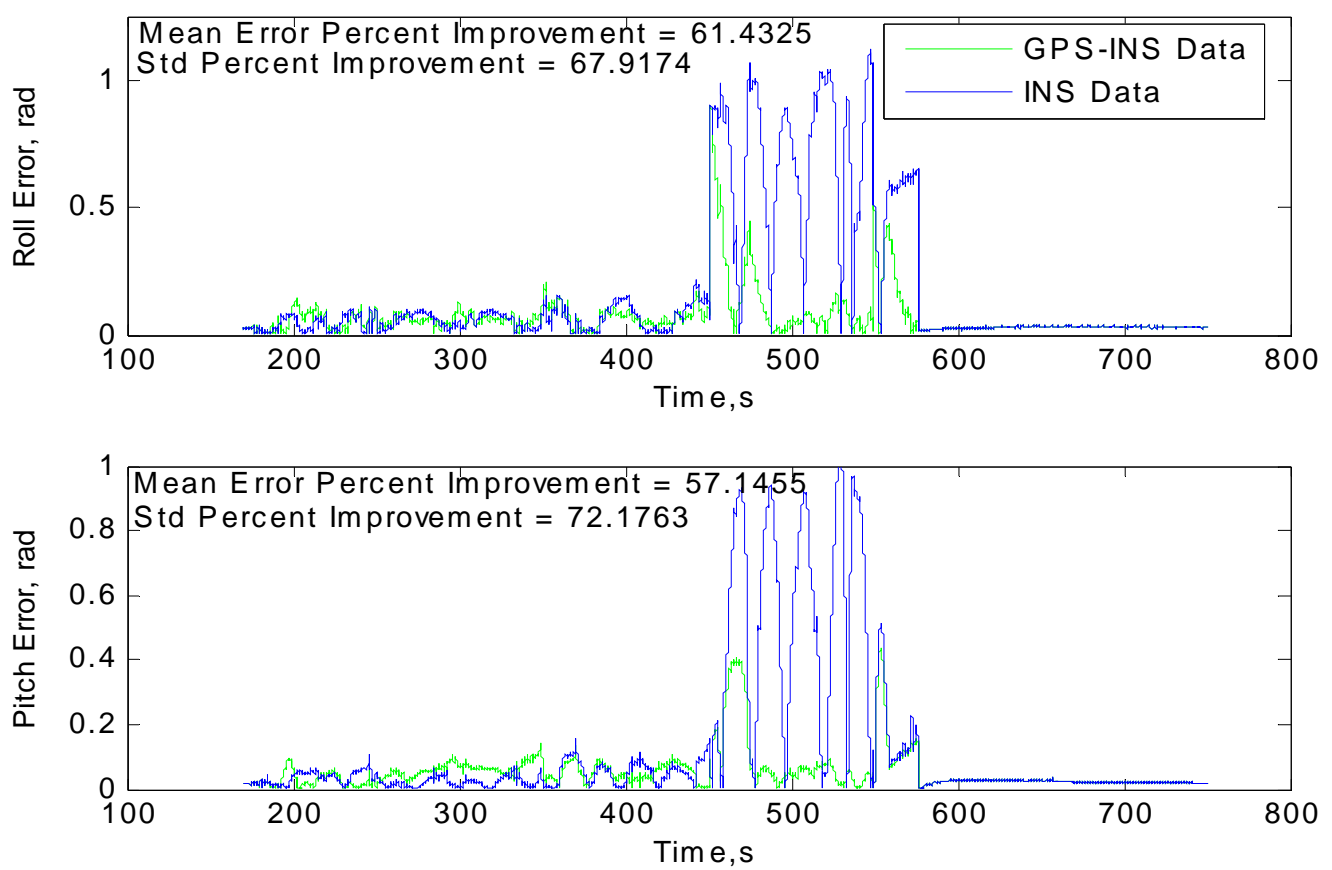

Figure C.4: Method IV Roll and Pitch Error (Validation Set 2) 